\title{
Tank Farm Surveillance and Waste Status Summary Report for November 1992
}

B. M. Hanlon

Date Published

February 1993

Prepared for the U.S. Department of Energy Office of Environmental Restoration and Waste Management

(2) Westinghouse $\begin{aligned} & \text { P.O. Box } 1970 \\ & \text { Hanford Company }\end{aligned}$

Hanford Operations and Engineering Contractor for the

U.S. Department of Energy under Contract DE-AC06-87RL10930 


\section{LEGAL DISCLAIMER}

This report was prepared as an account of work sponsored by an agency of the United States Government. Neither the United States Government nor any agency thereot, nor any of their employees, nor any of their contractors, subcontractors or their employees, makes any warranty, express or implied. or assumes any legal liability or responsibility for the accuracy, completeness, or any third party's use or the results of such use of any information, apparatus, product, or process disclosed, or represents that its use would not infringe privately owned rights. Reference herein to any specific commercial producl, process, or service by trade name. trademark, manufacturer, or otherwise, does not necessarily constitute or imply its endorsement, recommendation, or favoring by the United States Government or any agency thereof or its contractors or subcontractors. The views and opinions of authors expressed herein do not necessarily state or reflect those of the United States Government or any agency thereof.

This report has been reproduced from the best available copy. Available in paper copy and microfiche.

Available to the U.S. Department of Energy

and its contractors from

Office of Scientific and Technical Information

P.O. Box 62

Oak Ridge. TN 37831

(615) $576-8401$

Available to the public from the U.S. Department of Commerce National Technical Information Service

5285 Port Royal Finad

Springfield, VA 22161

(703) 487.4650

Printed in the United Stales of America

DISCLM-1.CHP $(1 \cdot 91)$ 
WHC-EP-0182-56

APPROVAL
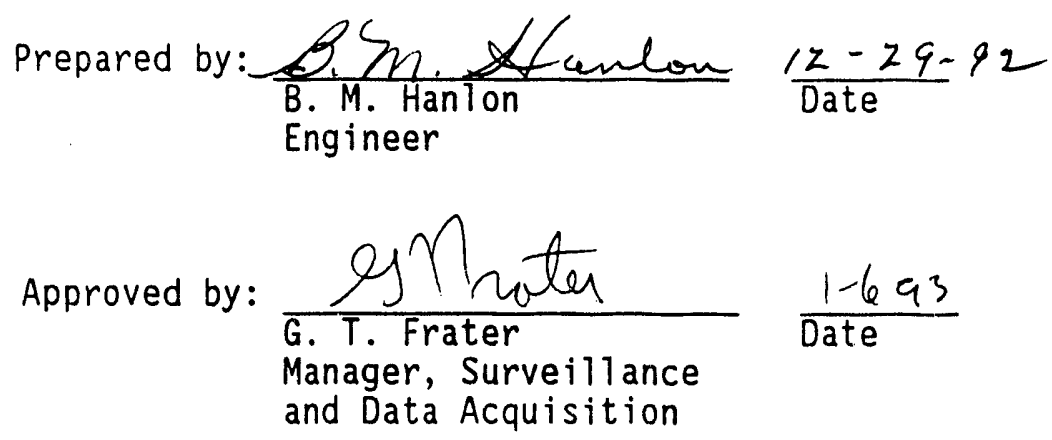


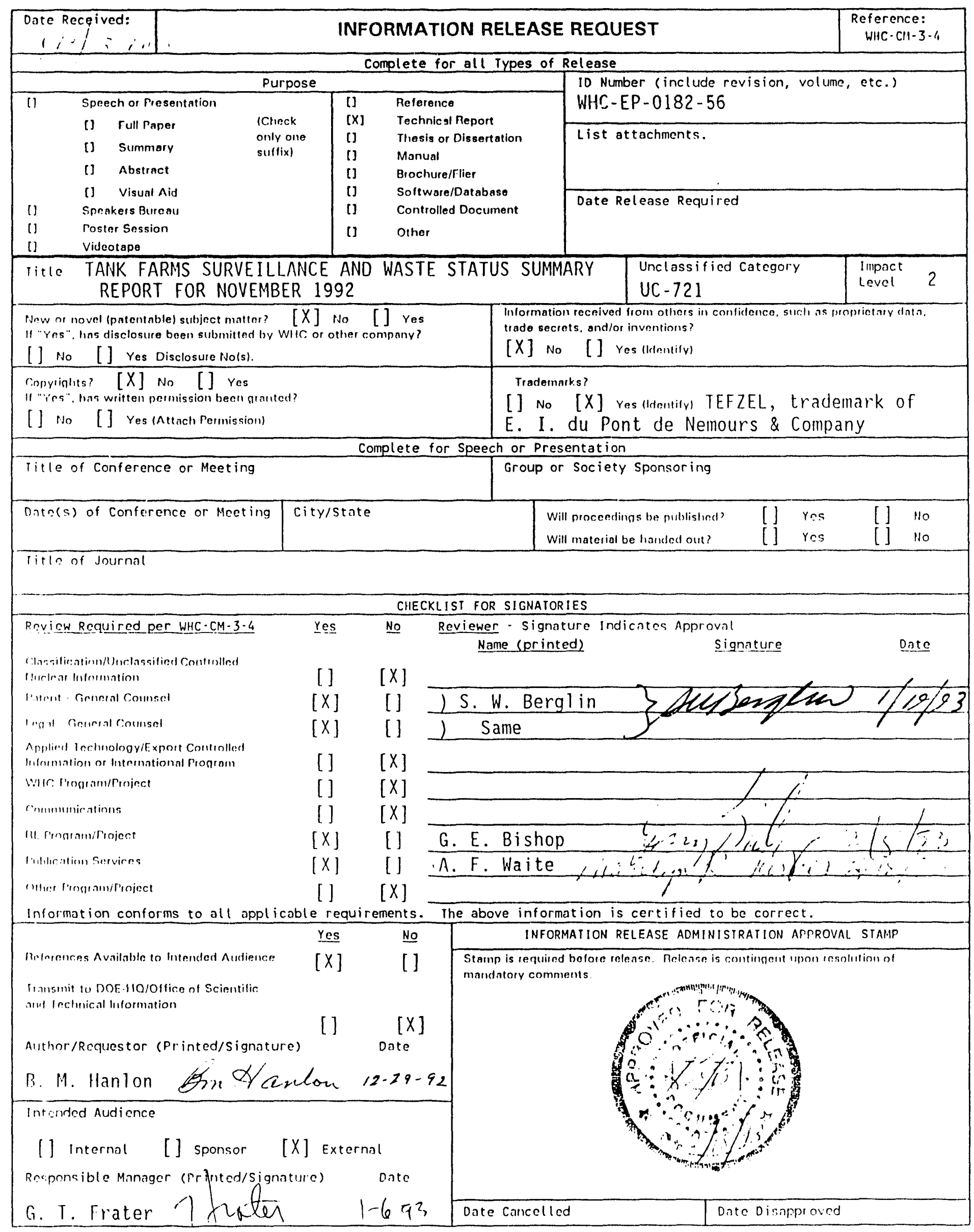


WHC-EP-0182-56

TANK FARM SURVEILLANCE AND WASTE STATUS SUMMARY REPORT FOR NOVEMBER 1992

\section{B. M. Hanlon}

\section{ABSTRACT}

This report is the official inventory for radioactive waste stored in underground tanks in the 200 Areas at the Hanford Site. Data that depict the status of stored radioactive waste and tank vessel integrity are contained within the report. This report provides data on each of the existing 177 large underground waste storage tanks and 49 smaller catch tanks and special surveillance facilities, and supplemental information regarding $\dot{t}$ ank surveillance anomalies and ongoing investigations. This report is intended to meet the requirement of $U$. S. Department of Energy-Richland Operations office Order 5820.2A, Chapter I, Section 3.e. (3) (DOE-RL, 1990, Radioactive Waste Management, U. S. Department of Energy-Richland Operation Office, Richland, Washington) requiring the reporting of waste inventories and space utilization for Hanford Tank Farm Tanks. 
WHC-EP-0182-56

This page intentionally left blank. 
SUMMARY

TANK STATUS

TANK INVESTIGATIONS

HIGHLIGHTS

Appendixes:

A. TANK AND EQUIPMENT CODE AND STATUS DEFINITIONS ............... A-1

Tank and Equipment Code/Status Definitions . . . . . . . . . . . A-3

B. TANK FARM CONFIGURATION, STATUS AND FACILITY CHARTS . . . . . . . . . . B-1

1 High-Level Waste Tank Configuration . . . . . . . . . . . . . . B-3

2 Double-Shell Tank Instrumentation Configuration . . . . . . . B-4

3 Single-Shell Tank Instrumentation Configuration . . . . . . . . B-5

4 Double-Shel1 Tank Status . . . . . . . . . . . . . . B-6

5200 E Single-Shel1 Tank Status . . . . . . . . . . . . . . B-7

6200 W Single-Shel1 Tank Status............... . . . . . . . .

7 Tank Farm Facilities Quick Reference . . . . . . . . . . . . . B-11

8 Hanford Tank Farms Facilities Chart . . . . . . . . . . . B-12

C. MONTHLY SUMMARY . . . . . . . . . . . . . . . . . . . . . . . C-1

1 Month1y Summary . . . . . . . . . . . . . . . . . . . C-3

2 Tank Use Summary . . . . . . . . . . . . . . . . . . C-4

3 Inventory Summary by Tank Farm . . . . . . . . . . . . . . . . C-5

4 Inventory and Status by Tank - Double-Shell Tanks . . . . . . . C-6

5 Inventory and Status by Tank - Single-Shell Tanks . . . . . . . . C-9

D. PERFORMANCE SUMMARY ........................ . D - . . . .

1 Performance Summary . . . . . . . . . . . . . . . . . D-3

E. Liquid STATUS AND PUMPABle liquid REMAINING IN TANKS . . . . . . . . . . . E- 1

1 Liquid Status and Pumpable Liquid Remaining in Tanks . . . . . . . E-3

F. PUMPING RECORD .......................... . F-1

1 Pumping Record...................... . . F-3

G. CATCH TANKS AND SPECIAL SURVEILLANCE FACILITIES . . . . . . . . . . . . G-1

1 East and West Area Catch Tanks and Special

Surveillance Facilities (Active). . . . . . . . . . . . . . G-3

2 East Area Catch Tanks and Special Surveillance

Facilities (Inactive) .. . . . . . . . . . . . . . . . G-4

3 West Area Catch Tanks and Special Surveiliance

Facilities (Inactive) ................. . G-5

H. LEAK VOlume estimates . . . . . . . . . . . . . . . . . . . . H-l

1 Single-Shell Tank Leak Volume Estimates . . . . . . . . . . . . . H-3 


\section{WHC-EP-0182-56}

This page intentionally left blank. 
1 FY 92 Integrated Core Sample Schedule . . . . . . . . . . . . 25

2 Current Status and Contingency Space for the 242-A

Evaporator Restart

\section{LIST OF TABLES}

1 Watch List Tanks . . . . . . . . . . . . . . . . . . . . . . . . . . 7

2 Tanks Containing $>1,000$ gram mole of Ferrocyanide

(Watch List Tanks) . . . . . . . . . . . . . . . . . . . . 8

3 Tanks with Potential for Hydrogen or Flammable Gas Accumulation

Above the Flammability Limit (Watch List Tanks) . . . . . . . . . . 13

4 Tanks Containing Concentrations of Organic Salts

$>3 \%$ Weight TOC (Watch List Tanks) . . . . . . . . . . . . . . . . 14

5 Single-Shell Tanks with High Heat Loads $(>40,000 \mathrm{Btu} / \mathrm{hr}) . . . . .15$

6 Double-Shell Tank Waste Type and Space Allocation . . . . . . . . . 18

7 Automatic Food Instrument Company (FIC) Gauges Out of Service . . . 19

8 Single-Shell Tanks Monitoring Compliance Status . . . . . . . . . . 20

9 Double-She11 Tanks Monitoring Compliance Status . . . . . . . . . . 24

\begin{tabular}{|l|l|l|}
\hline \multicolumn{3}{|c|}{ METRIC CONVERSION CHART } \\
\hline 1 inch & $=$ & 2.54 centimeters \\
\hline 1 foot & $=$ & 30.48 centimeters \\
\hline 1 gallon & $=$ & 3.80 liters \\
\hline 1 ton & 0.90 metric tons \\
\hline \multicolumn{2}{|c}{$\mathrm{F}=\left(\frac{9}{5}{ }^{\circ} \mathrm{C}\right)+32$} \\
\hline \\
$\begin{array}{r}\text { Btu/h }=2.930711 \text { E-01 watts } \\
\text { (International Table) }\end{array}$ \\
\hline
\end{tabular}


WHC-EP-0182-56

This page intentionally left blank 


\section{TANK FARM SURVEILLANCE AND WASTE STATUS SUMMARY REPORT FOR NOVEMBER 1992 SUMMARY}

Note: Changes from the previous month are in bold print.

TANK STATUS

\begin{tabular}{|l|c|c|}
\hline \multicolumn{1}{|c|}{ Category $^{|c|}$ Quantity } & Date of Last Change \\
\hline In-Service Tanks ${ }^{c}$ & 28 double-shell & $10 / 86$ \\
\hline Out-of-Service Tanks ${ }^{a}$ & 149 single-shell & $07 / 88$ \\
\hline Assumed-Leaker Tanks & 67 single-shell & $10 / 92$ \\
\hline Interim-Stabilized Tanks ${ }^{\text {b.d }}$ & 105 single-shell & $09 / 90$ \\
\hline Interim-Isolated Tanks $^{\text {e }}$ & 98 single-shell & $09 / 91$ \\
\hline
\end{tabular}

a All 149 single-shell tanks were removed from service (i.e., no longer authorized to receive waste) as of November 21, 1980.

${ }^{b}$ of the 105 tanks classified as interim stabilized, 58 are listed as assumed leakers.

"Six double-shell tanks 1isted as "in service" are currently not receiving waste because of inclusion on the Hydrogen Watch List and are thus prohibited from receiving waste in accordance with "Safety Measures for Waste Tanks at Hanford Nuclear Reservation," Section 3137 of the National Defense Authorization Act for Fiscal Year 1991, November 5, 1990, Public Law 101-510. Stabilized.

of the 48 single-shell tanks on Watch Lists, 21 have been Interim

e Of the 48 single-shell tanks on Watch Lists, 20 have been Interim Isolated.

\section{TANK INVESTIGATIONS}

Tank 241-SY-101. The surface level within this tank continues to fluctuate. The surface level increase/decrease phenomena has been observed since 1981, and is attributed to the slow buildup and relatively fast release of gas within the waste. An investigation into solutions to this slurry growth problem is ongoing. Multiple Event Fact Sheets, a Critique Report, Occurrence Reports, Discrepancy Reports, and Unusual Occurrence Reports have been issued. The Food Instrument Company (FIC) surface level monitor showed an increase from 405.10 in. on November 1 to 406.20 in. by November 30, 1992. The manual tape increased from 406.00 to 410.00 in. by November 30,1992 , (a new manual tape was installed on October 5,1992 , which is $10-7 / 8$ in. longer than previous manual tape). The radar gauge reading was $409.25 \mathrm{in}$. November 1 and 409.23 in. on November 30,1992 . The readings fluctuated between 408.75 and 409.90 in. during November. The surface level measurements for the radar gauge, FIC, and manual tape are different due to the location of the measurement devices, irregular surface, and the different method by which the level is obtained. 
Tank 241-SX-103. The level showed erratic increases/decreases from 1981 to March 1983. The level showed a decreasing trend from 247.90 in. in March 1983 to 242.10 in. on February 3, 1992. The FIC was out of service from February 10 to September 21, 1992. Following FIC repair on September 21 , the level was reading 240.00 in., increased to 241.00 in. on November 28, and was out of service November 29 and 30, 1992. An evaporation study will be performed by Waste Characterization Analys is to determine if the decrease can be attributed to evaporation. Liquid observation well (LOW) monitoring has been increased from quarterly to every two weeks. The ILL has remained stable at $20.5 \mathrm{ft}(-0.1 \mathrm{ft})$ from the established baseline since March 1, 1991, and was 1 ast scanned November 27, 1992. In-tank photographs taken December 17 , 1987 , show appears to be floating solids with some surface liquid. The FIC plummet is in a depression. Drywells are stable.

Tank 241-SX-105. The liquid observation well interstitial liquid level (ILL) showed a 0.1-ft decrease below basel ine when scanned August 6, 1991. The current scan of November 28, 1992, shows a $0.3-\mathrm{ft}$ decrease below baseline which is at the decrease criteria. An evaporation study was to have been performed by Waste Characterization Analys is to determine if the decrease could be attributed to evaporation. An evaporation study cannot be performed for tank 105-SX because of the complete solid surface of the tank. At the present time, there is no computer model available for determining evaporation rates for tanks with a $100 \%$ solid surface. LOW monitoring has been increased from quarterly to every two weeks. In-tank photographs taken June 15, 1988, show a dry cracked, uneven surface. The FIC is in the intrusion mode of operation, and the FIC plummet is suspended over solids. Work is in progress to repair the radiation detection laterals underneath this tank to provide improved leak detection.

\section{Potential or Assumed Leaks:}

Tank 241-T-101. A surface level decrease of $2.60 \mathrm{in}$. from the previously established liquid level of 44.20 in. was noted on September 18, 1992, following maintenance on the 101-T Level Indicator (FIC). Because the FIC had been operating only sporadically since December 1991, and no reliable surface level data was obtained between December 1991 and July 1992, the decrease was assumed to be due to the faulty FIC, and no occurrence reporting action was taken during that time. Drywell scan data was reviewed and is stable. Drywell monitoring frequencies have been increased to weekly. The surface level measurement was 41.50 in. on October 31,1992 , and increased to 43.80 in. after a reference elevation check was performed by instrument technicians on November 14, 1992. The level is reading 43.60 in. on November 30. Occurrence Report RL-WHC-TANKFARM-1992-0073 was issued October 1, 1992, and Tank 101-T was declared an assumed leaker on October 4, 1992. Drywell scan data was reviewed and is stable. Drywell monitoring frequencies have been increased to weekly. Plans are underway to pump this tank.

\section{Potential or Assumed Intrusions:}

The following tanks have been reported for potential in-leakage (intrusions) from known/unknown sources.

Tank 241-B-202. A steady increase in the surface level measurement has been observed since December 1984. The manual tape pencil plummet is contacting liquid. When the quarterly reading was obtalned on October 6, 1992, the level 
was recorded as 144.75 in., thus exceeding the 2.00-in. increase criteria from the established baseline of 142.50 in. The surface level measurement was rechecked on October 9, 1992, (145.50 in.) and October 13 (145.00 in.), verifying the increase and that the criteria had been exceeded. The monitoring frequency has been increased from quarterly to monthly. The reading for November 23, 1902, was 145.00 in. Tank 202-B has been added to the Alert List and $w:-1$ be reported in accordance with applicable reporting requirements.

Tank 241-S-107. A slow increase in the surface level has been observed since May 1987. The surface level measurement increased 1.10 in. in September 1991, when water was added to the tank in order to install a saltwell screen. The reference baseline was adjusted to reflect this water addition. On August 3 , 1992, the surface level measurement was $144.50 \mathrm{in.}$, thus exceeding the 2.00-in. increase criteria from the established baseline of $142.40 \mathrm{in}$. The FIC was reported out of service on August 17, 1992. After the FIC was repaired on September 21 , the surface level measurement was 144.20 in., which is within criteria limits. The reading remained at the $144.20-i n$. level to November 30, 1992. This tank will remain under close surveillance for additional unexplained surface level increases.

Tank 241-TX-115. The LOW scans revealed an ILL increase over baseline in excess of the establ ished $0.4-\mathrm{ft}$ increase criteria in May 1987. Comparison of past and present in-tank photographs show no sigrificant change in surface conditions or obvious evidence of intrusion. Environmental Protection

Deviation Report \#88-08 was issued on May 18, 1988. Event Fact Sheet \#TF-EFS80-140 was issued January 9, 1990. The ILL showed an additional increase of $0.4 \mathrm{ft}$ on March 1, 1991. The increase was verified on March 11, 1991. These LOWs are monitored quarterly, alternating every six weeks with the neutron and gamma probes. No further increase of the ILL has been observed since March 1 , 1991. The LOW was 1ast scanned with the neutron probe on October 23, 1992.

Tank 241-TY-102. On November 8, 1992, the automatic FIC surface level measurement of 32.10 in. exceeded the 0.50 -in. increase criteria from the reference baseline of 31.40 in. Discrepancy Report S\&DA-92-489 was issued November 9, 1992. The tank has a history of icicle buildup on the FIC plummet and intrusions. A request was made on November 16 for Tank Farm Operations to flush the FIC plummet. Work Order 2W-92-01155 was written to perform the flush, scheduled for December 3, 1992.

\section{HIGHLIGHTS}

\section{Tank 241-AW-101}

On October 21, 1992, the surface level decreased and the pressure rose in Tank 241-AW-101. A review of several tanks in the AN, AP, AW and U Tank Farms for potential for generation, retention and release of flammable gases was conducted in 1990. As a result of the new level decrease and pressure increase data, Tank 241-AW-101 was reevaluated and placed on the Hydrogen Watch List (potertial for flammable gases) on November 5, 1992. (See Item 4, Occlirrenre Report WHC-TANKFARM-1992-0083 for details). 


\section{Criticality Safety Issues}

On April 30, 1992, an Unreviewed Safety Question concerning criticality safety issues in the Tank Farms was declared to be a reportable event. Unusual Occurrence report RL-WHC-TANKFARM-1992-0037 was issued. A prohibition was placed on all waste transfers into and between the tank farm facilities which is negatively affecting various Hanford programs. On September 1, 1992, the approved Justification For Continued Operations (JCO) was received. This JCO establishes the 1 imitations for all tanks receiving transfers and also excludes any stabilization of single-shell tanks until further evaluations are completed and approved by DOE-HQ. The resolution of the USQ is scheduled for summer 1993.

\section{Saltwell Pumping}

Interim stabilization has been delayed because of the criticality safety issues which suspended waste transfers into and between tank farm facilities. The approved Justification For Continued Operations (JCO) excludes any stabilization of single-shell tanks until further evaluations are completed and approved by DOE-HQ. The resolution of the USQ is scheduled for summer 1993.

4. Occurrence Reports

RL-WHC-TANKFARM-1992-0082 (UNUSUAL OCCURRENCE) - LOSS OF LEAK DETECTION CAPABILITY FOR TANK 241-SY-102; A NON-CONFORMANCE TO A SAFETY DOCUMENT LIMITING CONDITION OF OPERATION (LCO) RESULTS. (10-Day Report)

A loss of both leak detection systems for double-shell tank 241-SY-102 initiated this event report. On November 1 , it was discovered the annulus continuous air monitor (CAM) was not functional because of a previously failed vacuum pump. The annulus leak detection probe was verified as operable. On November 2, a priority 1 work package was initiated to replace the vacuum pump. The first repair attempt was not successful. The repair attempts continued until the second leak detection method failed.

On November 4, it was determined the annulus flake box leak detector probe was not operable. This condition resulted in a non-conformance to a Limiting Condition of Operation (LCO) for OSR-T-152-00001 (Section 11.4), which requires at least one operable leak detection system. A priority 1 work package was initiated. On November 4 , the annulus leak detection probe was reset and verified operable, and the vacuum pump in the annulus CAM cabinet was repaired.

Annulus leak detection capability was lost for less than one eight hour shift, and alternative monitoring was performed until leak detection was restored. Both systems were expeditiously restored on a priority basis. There was not significant impact on the plant or program as a result of this incident. 
RL-WHC-TANKFARM-1992-0083 (OFF NORMAL) - TANK 241-AW-101 EXPERIENCES SURFACE LEVEL DROP AND VENT SYSTEM PRESSURE INCREASE; THE TANK HAS BEEN ADDED TO THE HYDROGEN WATCH LIST. (Latest 10 Day Report)

On October 21, 1992, the surface level decreased 1.5 in. and the tank pressure rose approximately 0.9 inches of water, from -1.8 to -0.9 in. water gauge. The other tanks in the AN Tank Farm showed smaller pressure increases of about 0.3 in. The pressure gradually returned to normal in about five minutes. The apparent venting was noted because of a larger than normal level drop accompanied by a brief fluctuation in tank pressure. A review of the 241-AW-101 tank data was initiated to determine if it should be added to the flammable gas watch 1 ist. on November 5, it was decided to place this tank on the Flammable (Hydrogen) Gas Watch List.

In 1990, a review of 102-AN, 107-AN, 105-AP, 101-AW, and 111-U tanks that may have a potential for the generation, retention and release of flammable gases was conducted. Oniy Tank 101-AW was added to the Watch List; the other tanks did not exhibit the requisite characteristics. Tank 101-AW was added because of the new pressure data. As new data become available in the future, it is possible that additional tanks may be placed on the Watch List.

The addition of this tank to the Hydrogen Watch List may require additional program funds for resolution of the safety issue, and will require additional funds for performing maintenance on the tank and related ventilation equipment. This tank had previously been identified to be the second tank processed through the Grout Facility. Identification of this tank as a Watch List Tank could impact the schedule for the grout campaigns.

RL-WHC-TANKFARM-1992-0086 (OFF NORMAL) - INTERSTITIAL LIQUID LEVEL MEASUREMENTS DECREASE TO ESTABLISHED BASELINE $(.3$ FT) IN SINGLE-SHELL TANK 241-SX-105). (10-Day Report)

On October 9, 1992, as a result of Liquid Observation Well (Low) monitoring the interstitial liquid level (ILL) for 241-SX-105 was determined to have reached the $.3-\mathrm{ft}$ decrease 1 imit over a five year period.

A preliminary statistical analysis of the variability and significance of deviations of the ILL was completed on November 16, 1992. The analysis concluded that the measured drop in liquid level was statistically significant. Further analysis of the stability of the ILL data over time is continuing.

This tank is an actively ventilated single-shell tank that has not been stabilized. It contains approximately 261,000 gal of drainable liquid in about 683,000 gal of total waste. Photographs taken in 1988 show that the surface of the waste is a dry crust. This crust prevents the effective use of a conductivity-based liquid level device. Therefore, the LOW is considered to be the primary means of monitoring ILL level in the tank. 
Tank 241-SX-105 is a Hydrogen Watch List tank because it has the potential to generate hydrogen gas. As a result of this designation, additional precautions are required when performing work on the tank and associated ventilation system. This has the potential to delay stabilization activities, and even routine maintenance.

Quarterly readings with neutrons and gamma probes are required, with the neutron probe being the primary instrument for measuring ILL. The neutron probe LOW readings were taken on a quarterly basis until November 6, 1991. Because of work restrictions as a result of vapor issues and neutron probe failures, neutron probe LOW readings were not taken again until 0ctober 9, 1992. Neutron probe LOW readings are now being taken on a bi-weekly basis. Gamma probe LOW readings continue on a quarterly basis.

The preliminary engineering analysis has not conclusively determined the cause of the decrease. Drywell readings associated with 105-SX have not indicated any evidence of a leak. Temperature readings are normal for this tank. It is possible that the decreasing trend in liquid level is at least partially due to evaporation. Work continues to determine if an appropriate model is available for evaporation analysis. The evaporation analysis is complicated by the fact that the waste is sovered by a hard surface crust which acts as a barrier. Current modeling methods are not designed to account for a solid surface.

\section{Changes to the Report:}

\section{SUMMARY}

Table 9 (Double-Shell Tanks Monitoring Compliance Status) has been added to the report. This table indicates whether double-shell tank monitoring is in compliance with the specified requirements. 


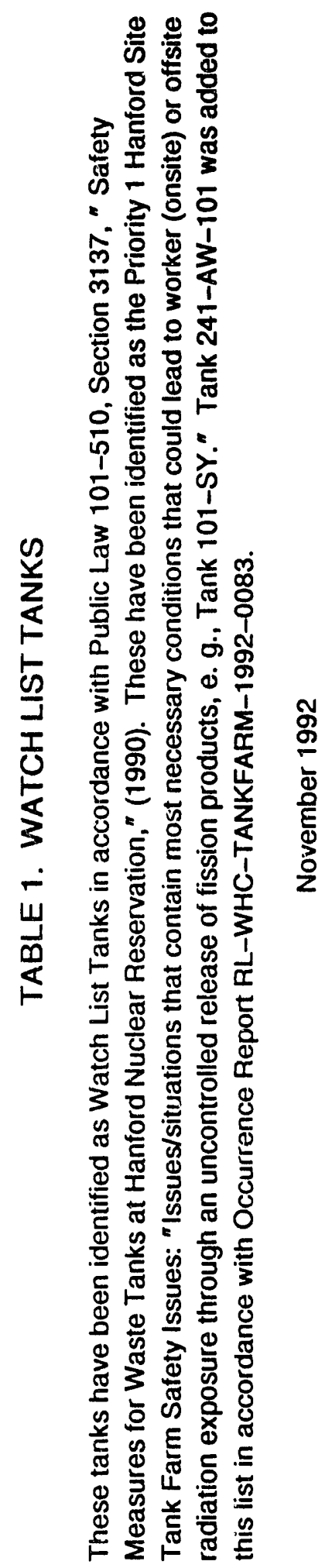

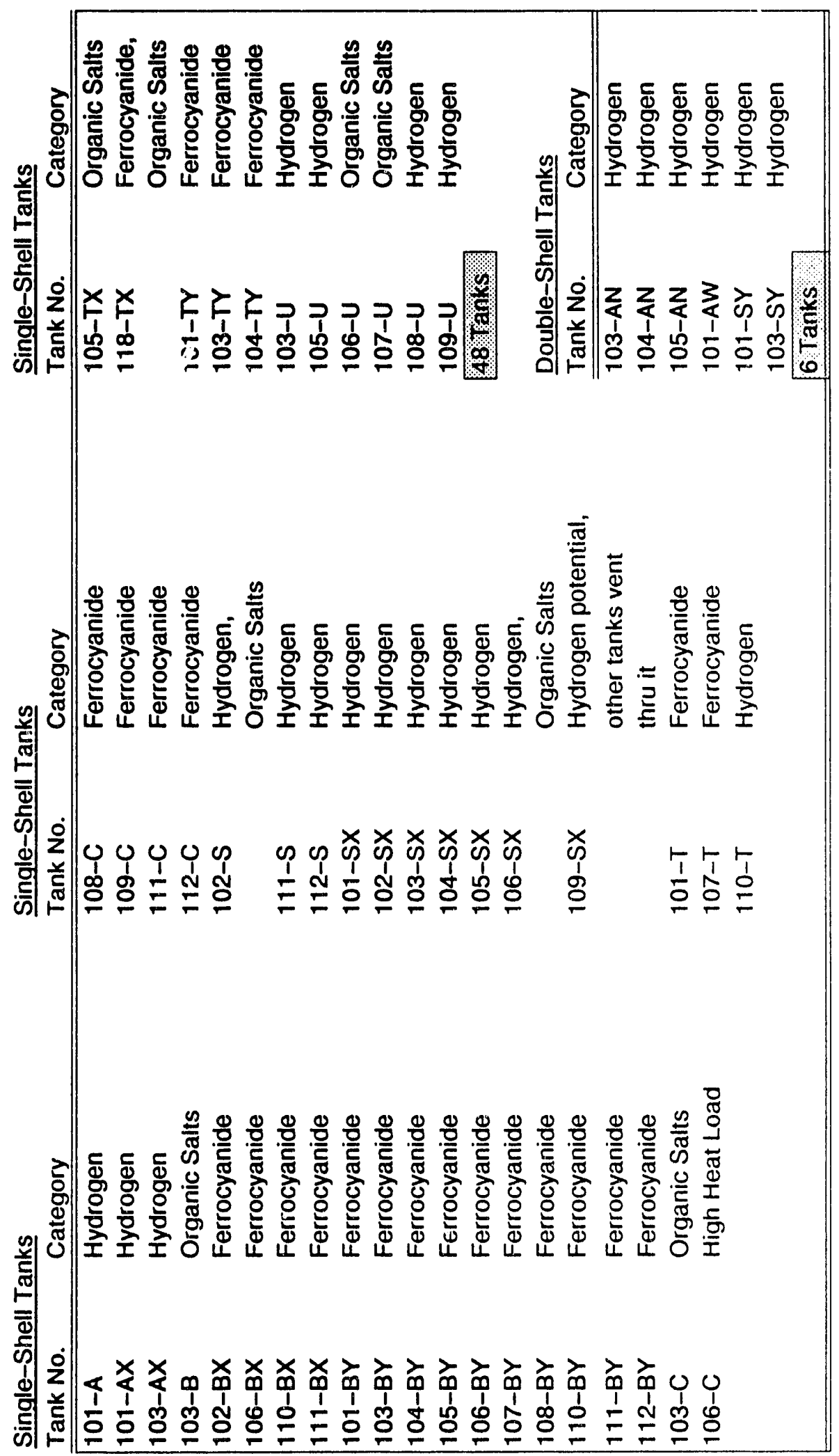



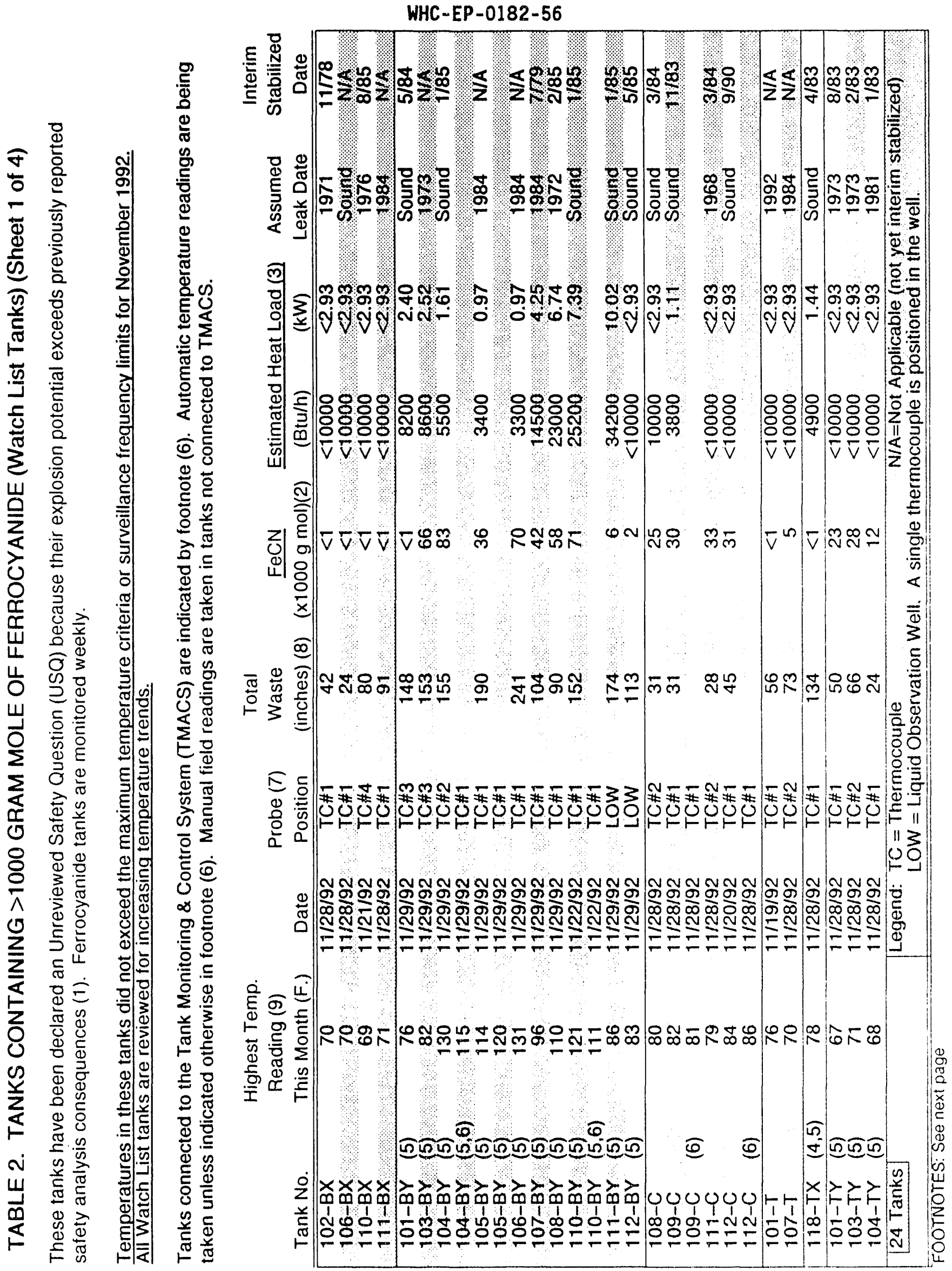


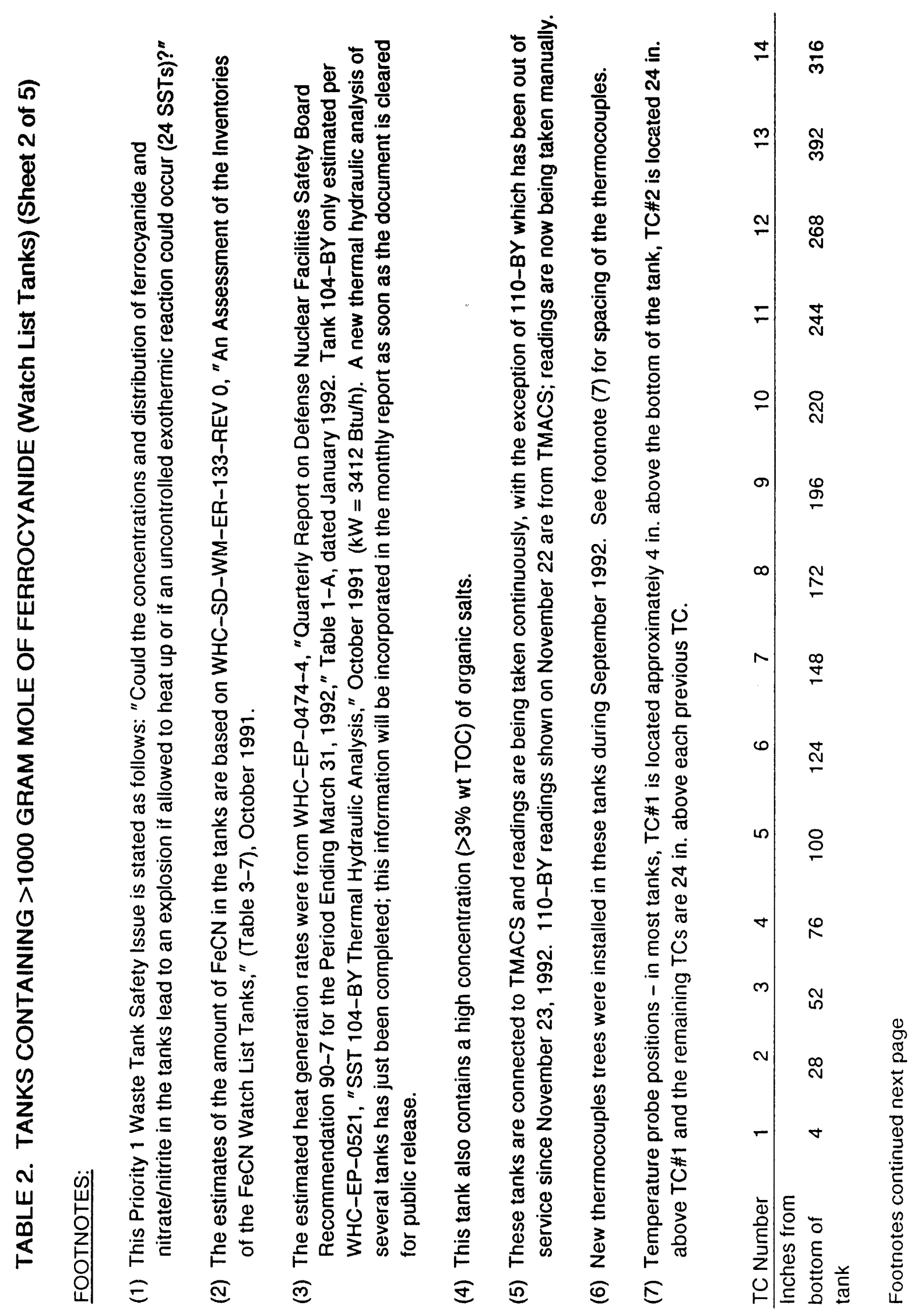




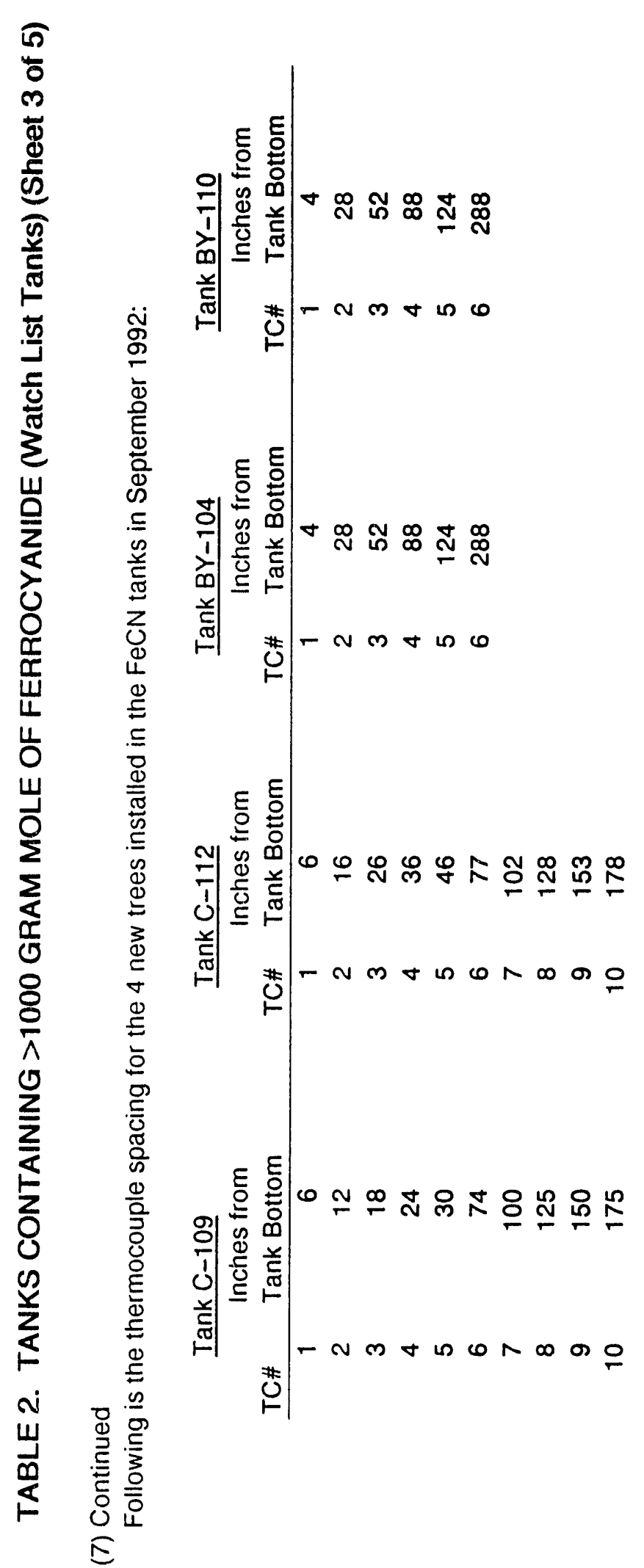

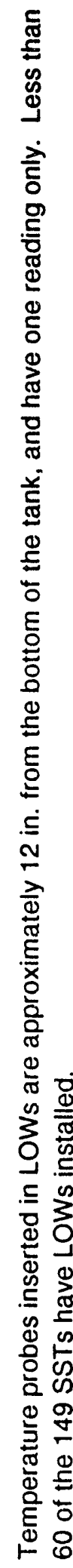

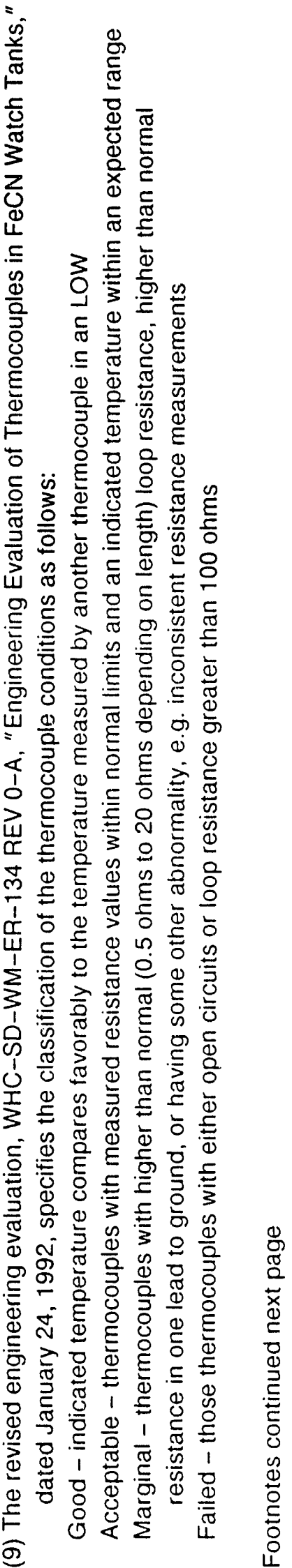




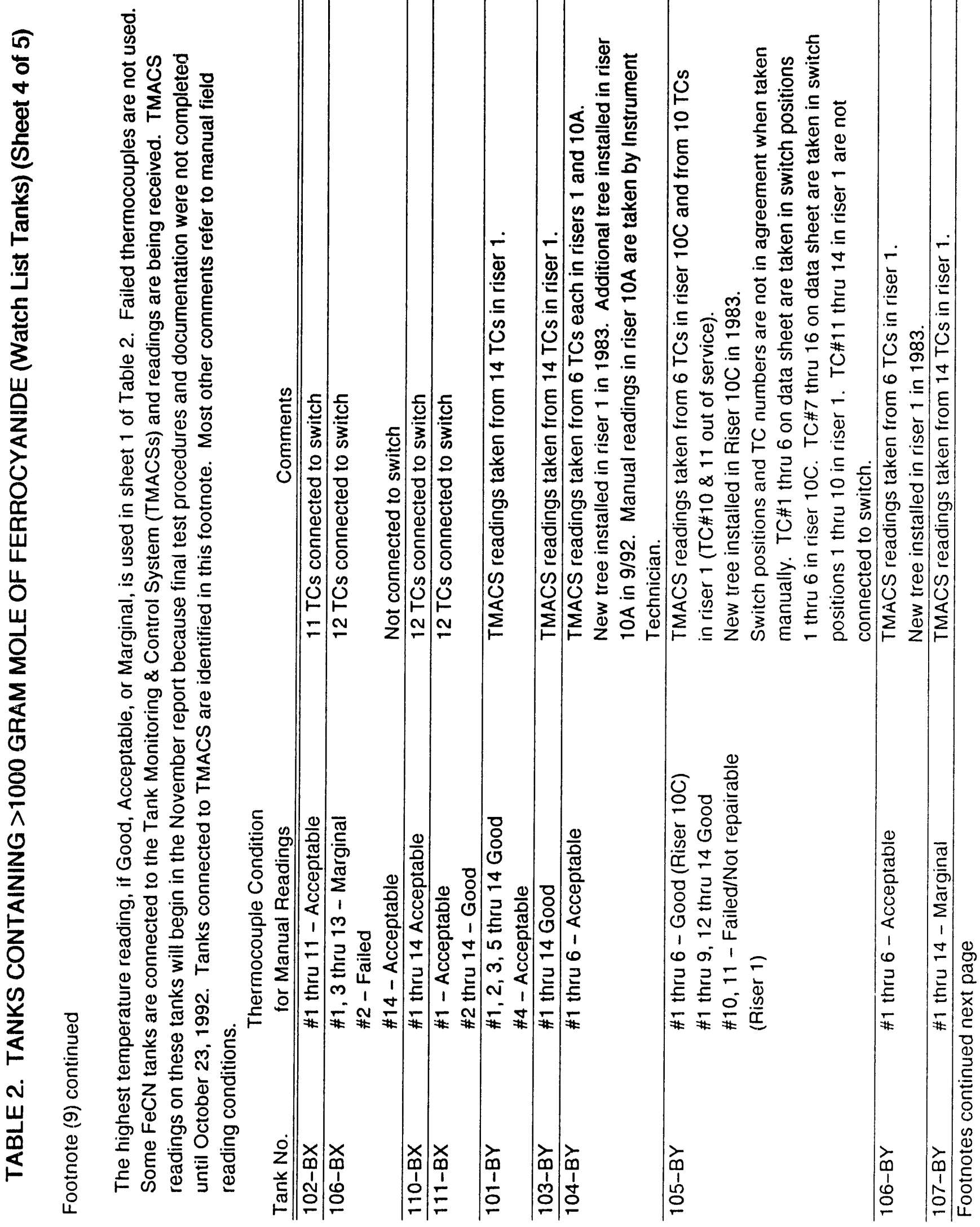




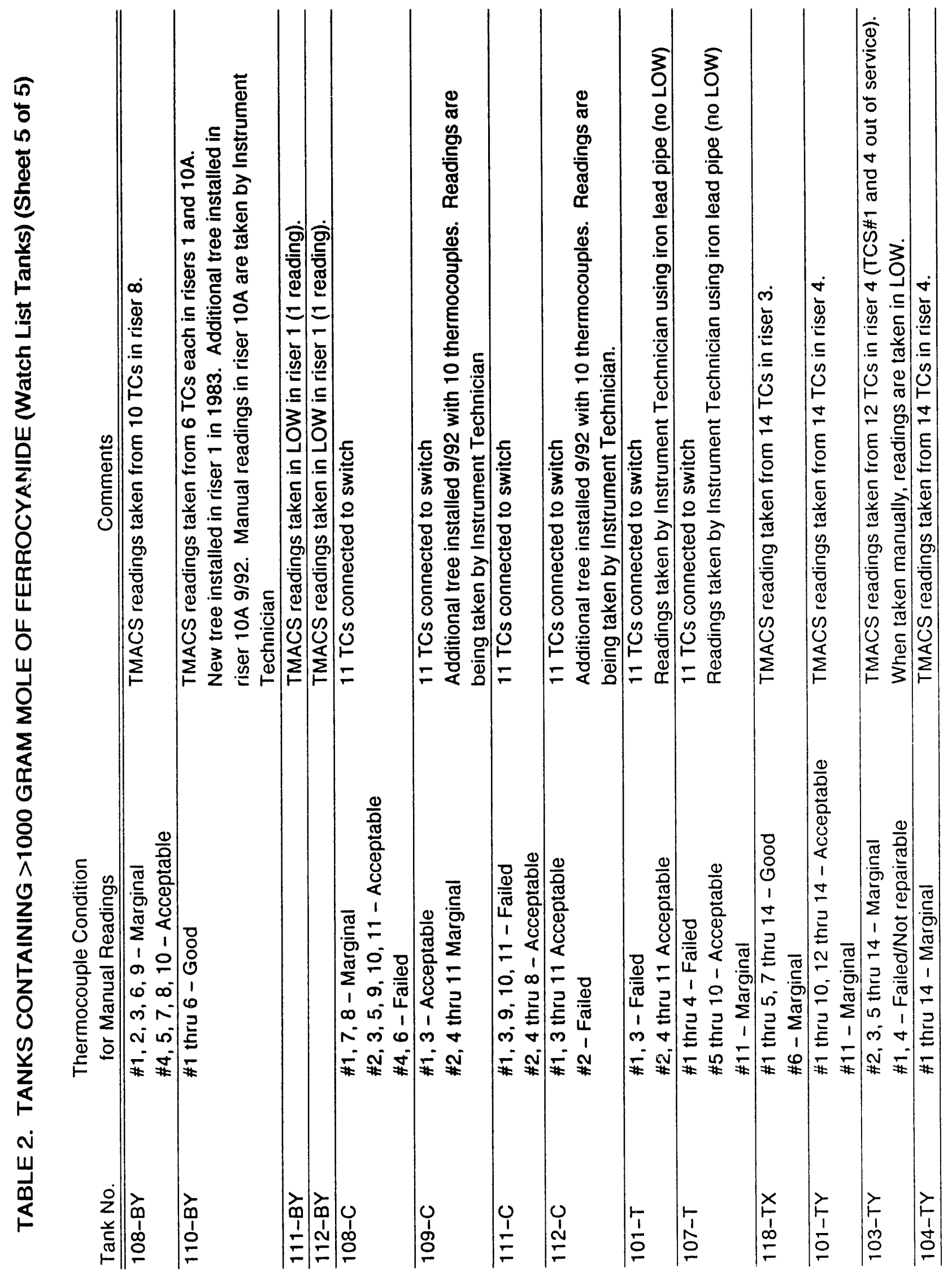



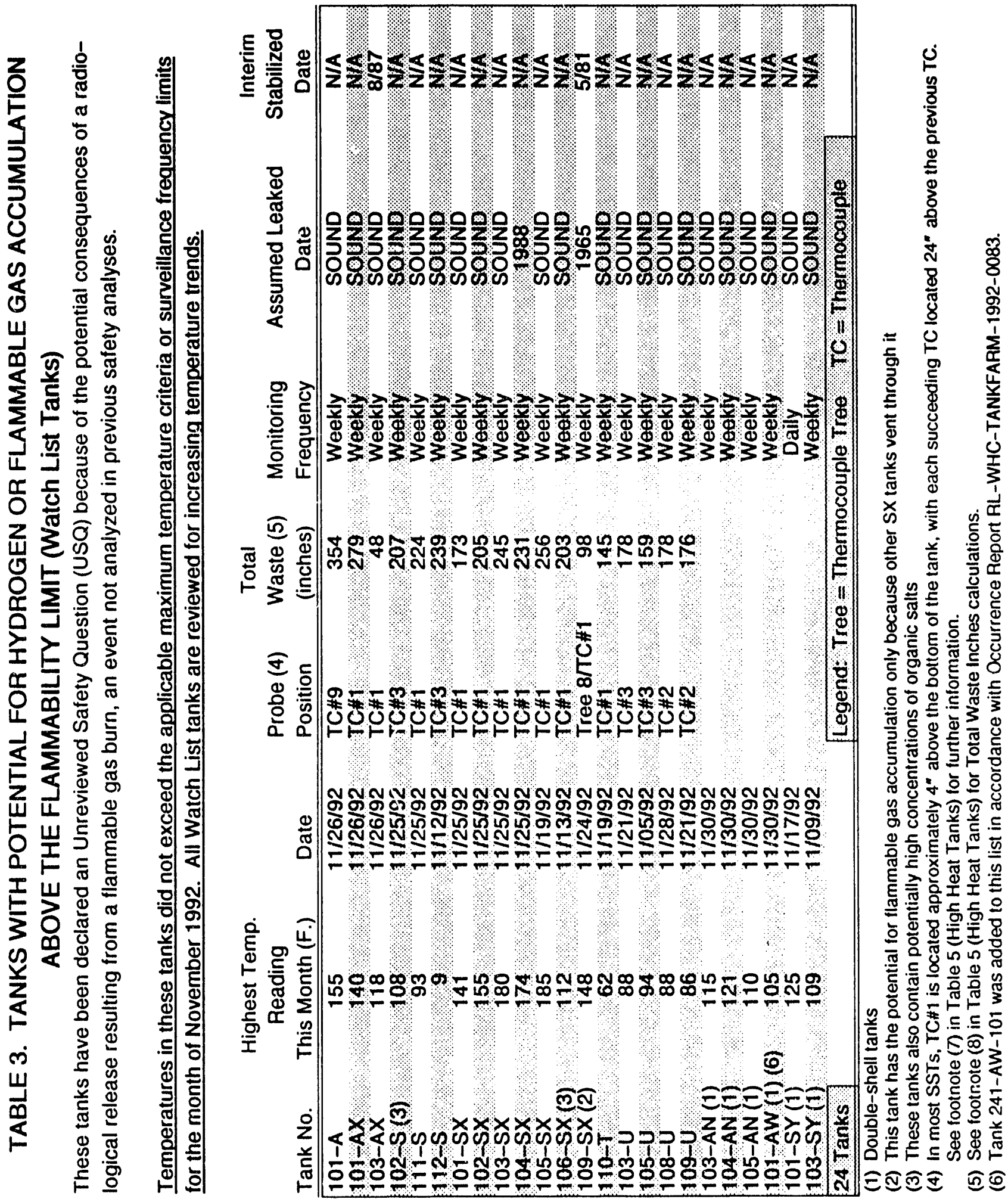

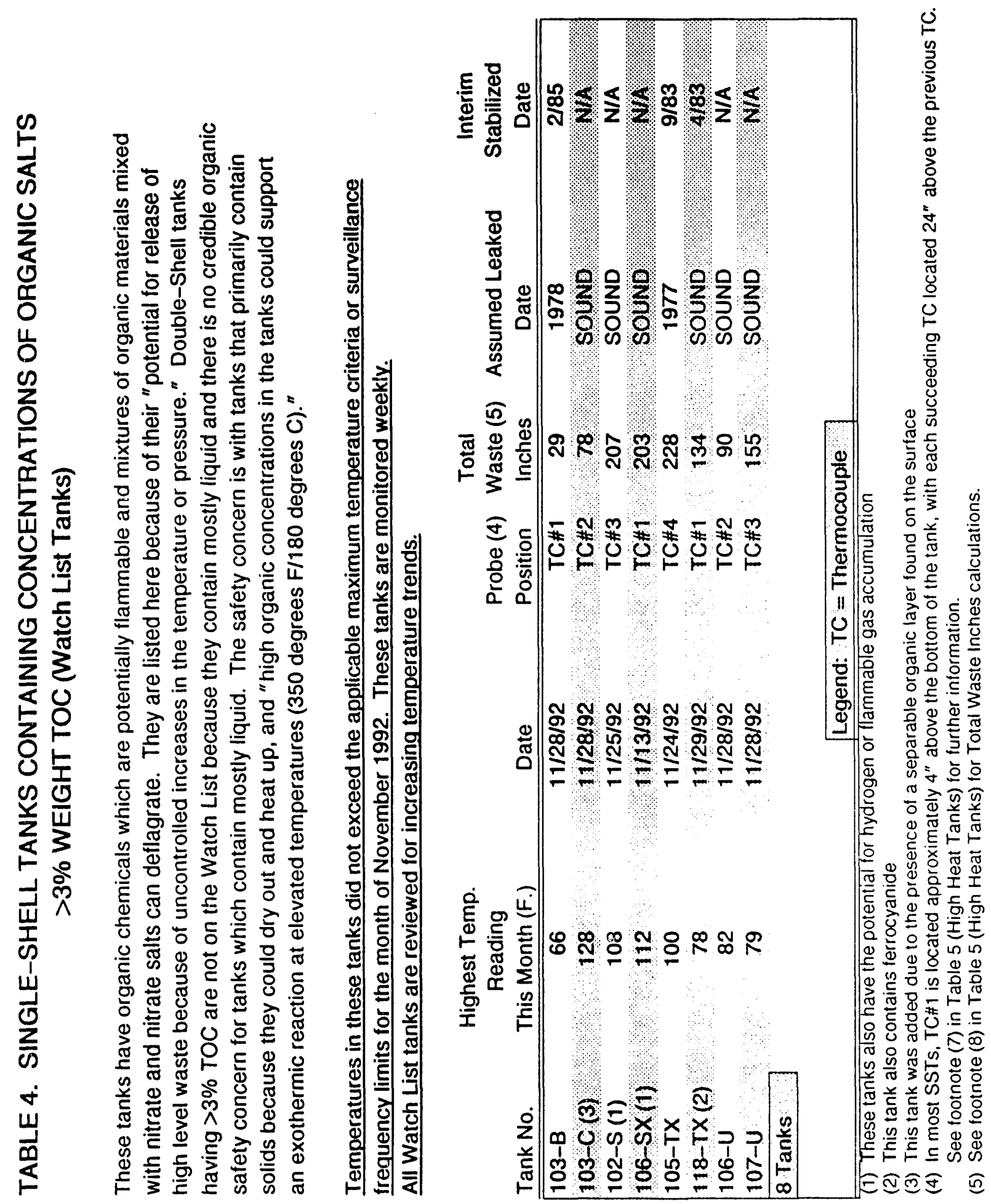


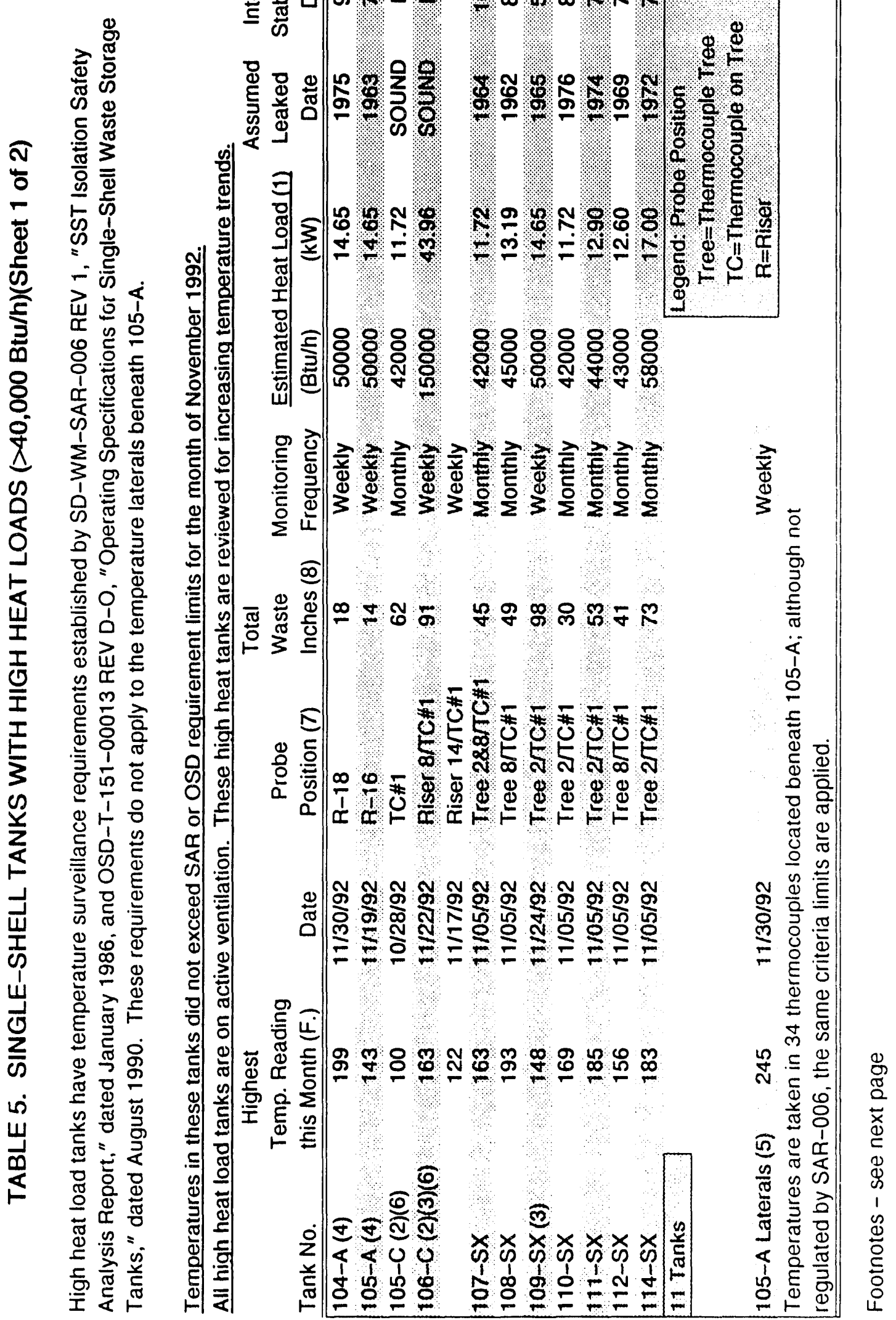




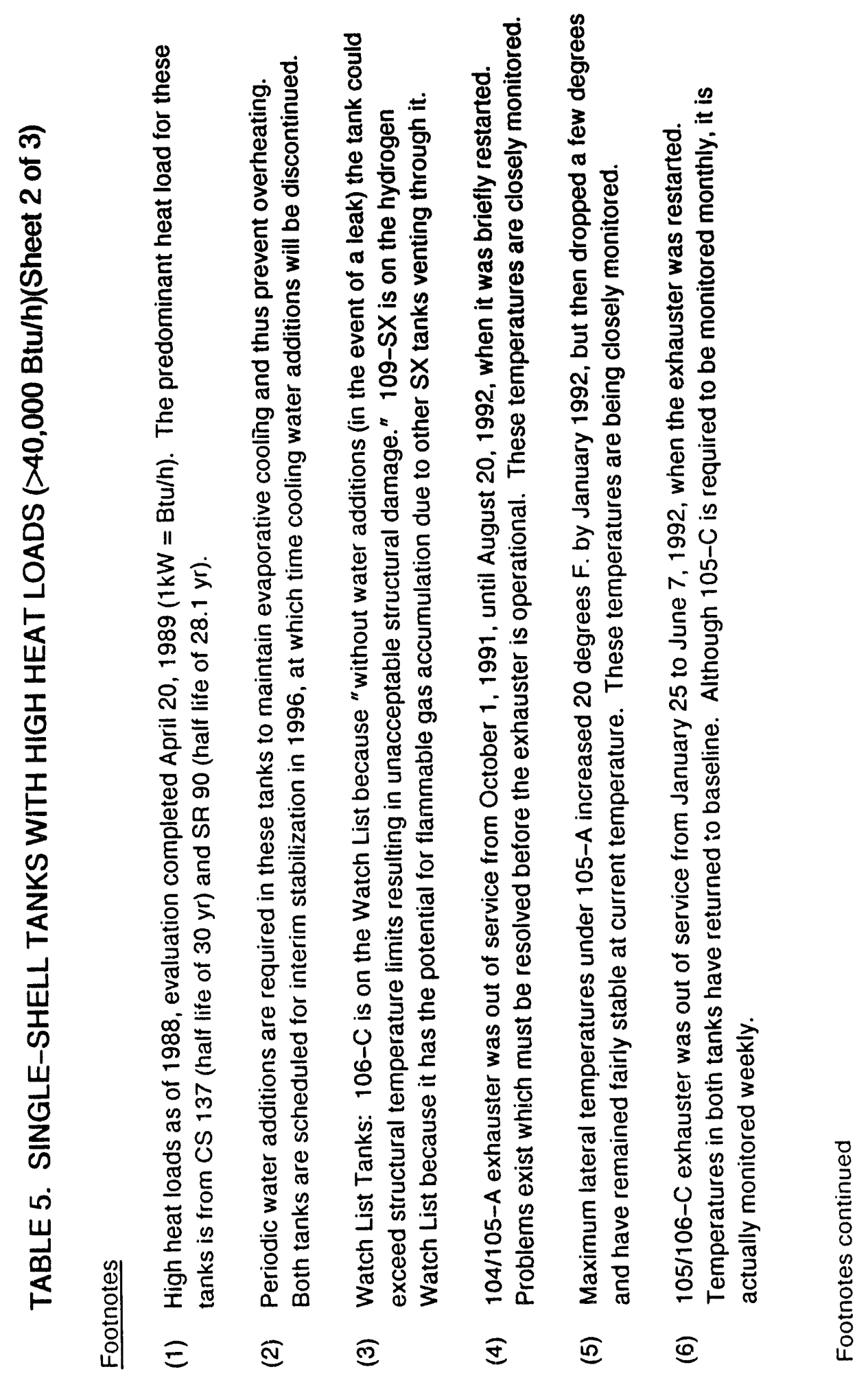




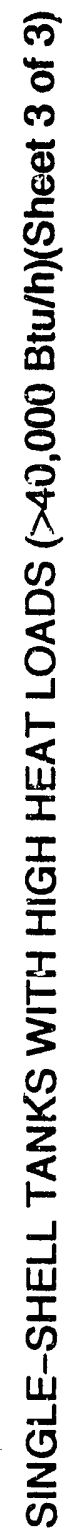

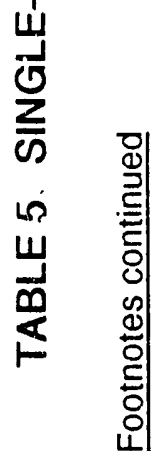

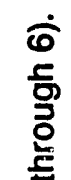

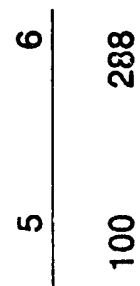

.

$\frac{x}{\omega}$.

둥

< $\frac{3}{5}$

을

就

兽苋

.

兽 $\frac{0}{5}$

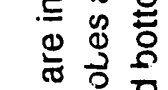

乐造造

내응 을

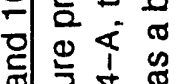

สิ

过这

의

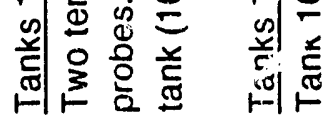

E

$m$ กิ

N $\stackrel{\infty}{\sim}$

U)

읜

ㅎำ

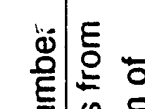
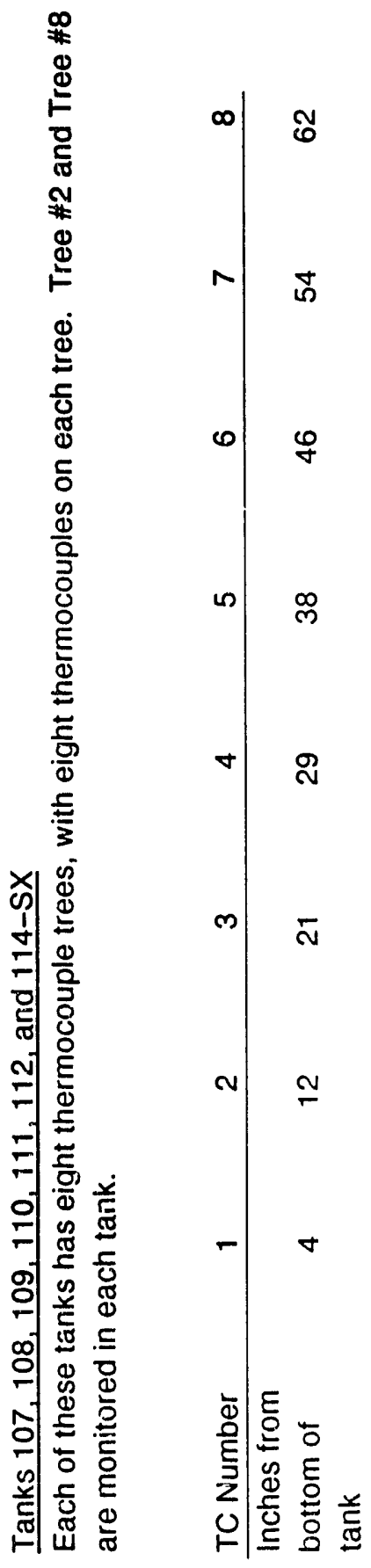

$*$
d
$\frac{E}{U}$
$\stackrel{ }{N}$
$\frac{N}{+}$

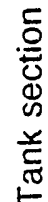

*

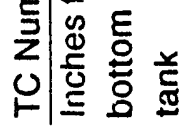

矛苋
莡

을

产

든

ज

흥

ह

용 ह

을

능

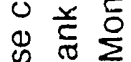

$\stackrel{0}{\Xi}$

巨.

ฏ

$\leqq \varepsilon$

ㅇㄴㅇ

हू ญ

战

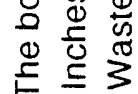




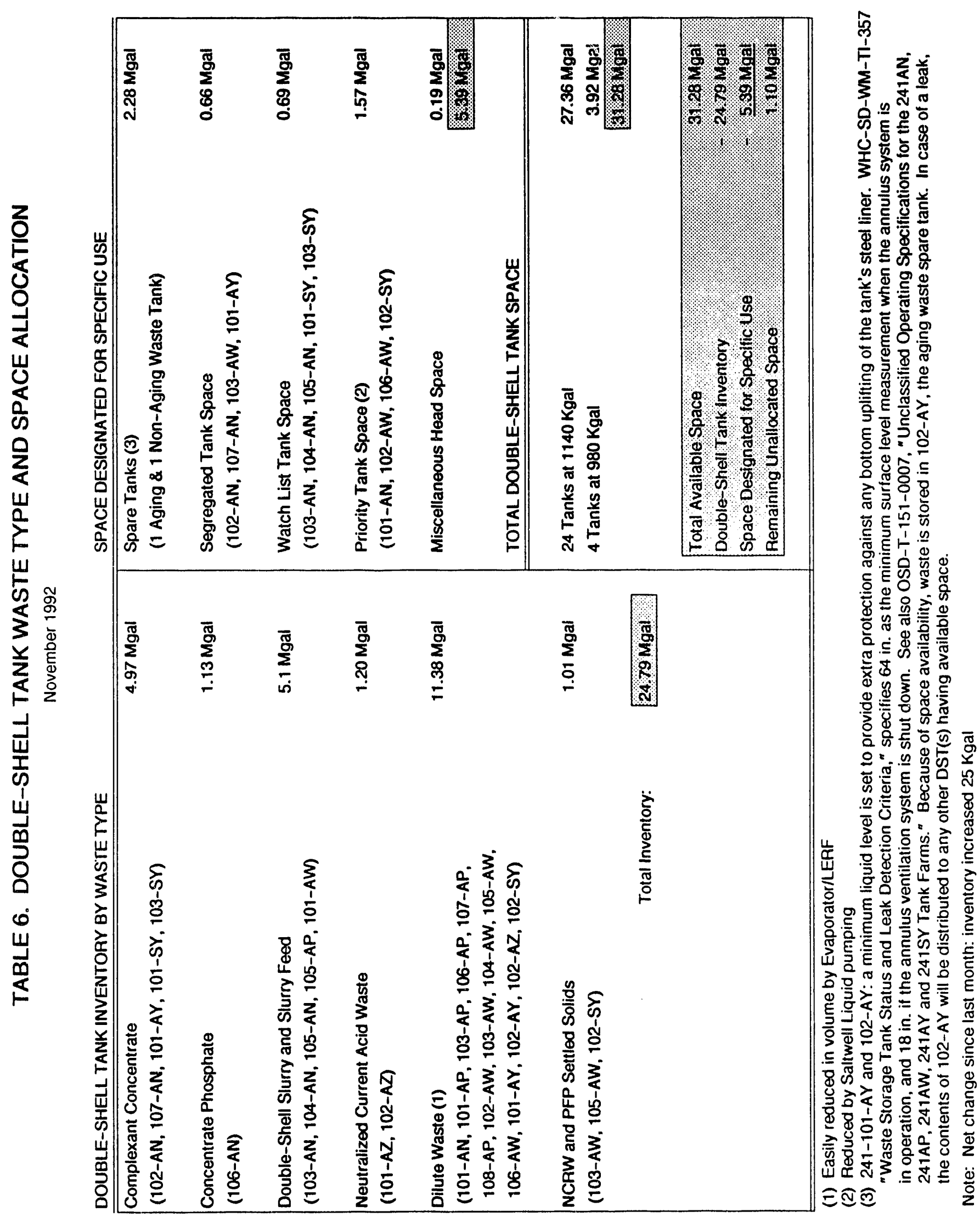




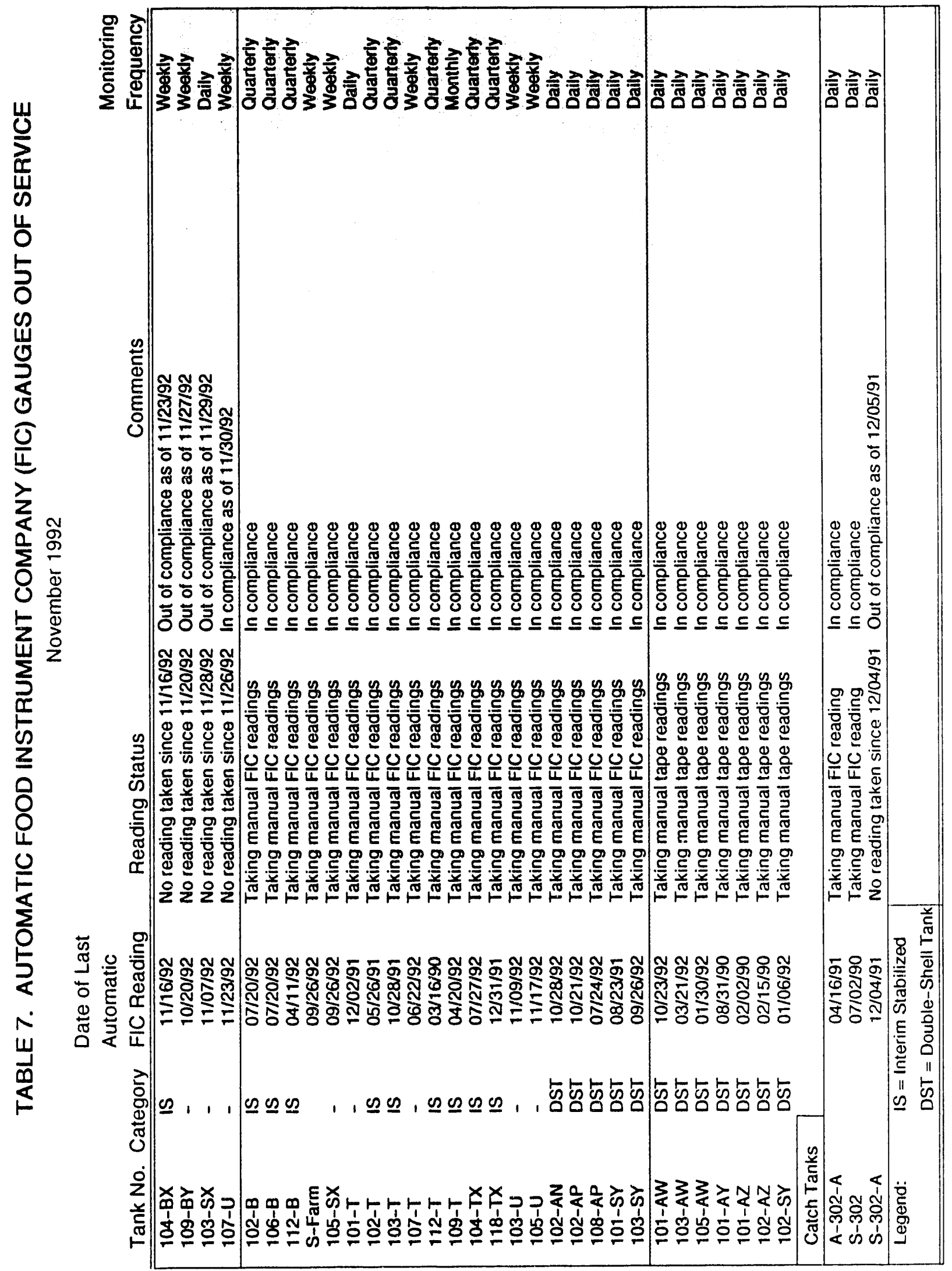




\section{TABLE 8. SINGLE-SHELL TANKS MONITORING COMPLIANCE STATUS 149 TANKS (Sheet 1 of 4)}

The following table indicates whether Single-Shell tank monitoring is in compliance with the requirements as specified in the applicable documents:

NOTE:

All Watch List and High Heat tank temperature monitoring is in compliance.

All Dome Elevation Survey monitoring is in compliance.

All Drywell monitoring is in compliance.

Psychrometrics (2)

In-tank Photographs (3)

\begin{tabular}{|c|c|}
\hline $\begin{array}{l}\text { LEGEND: } \\
(1-\% 100)\end{array}$ & - In compliance with all applicable documentation \\
\hline OK & $=$ Out of compliance with documentation \\
\hline-357 & $\begin{aligned}= & \text { WHC-SD-WM-TI-357. "Waste Storage Tank Status and Leak } \\
& \text { Detection Criteria" }\end{aligned}$ \\
\hline POP & $\begin{array}{l}\text { - Plant Operating Procedure TO-040-650, "Obtain/Record SST } \\
\text { Temperatures" }\end{array}$ \\
\hline M.T. & = Manual Tape \\
\hline FIC & $=$ Food Instrument Company \\
\hline OSP/SAR & $\begin{array}{l}=\text { Operations Safety Requirements/Safety Analysis Report } \\
\text { Requirements }\end{array}$ \\
\hline OSD & = Operating Specifications Document \\
\hline N/A & $=$ Not Applicable (i.e., no LOW. M.T, FIC installed) \\
\hline o/s & $=$ Out of Service \\
\hline Gamma & $\begin{array}{l}=\text { LOW readings taken by Gamma probe } \\
=\text { LOW readings taken by Neutron probe }\end{array}$ \\
\hline
\end{tabular}

Information as of $11 / 30 / 92$

\begin{tabular}{|c|c|c|c|c|c|c|c|c|c|c|}
\hline \multirow{4}{*}{$\begin{array}{l}\text { Tank } \\
\text { Number }\end{array}$} & \multirow{2}{*}{\multicolumn{2}{|c|}{ Category }} & \multirow{4}{*}{$\begin{array}{l}\text { Temperature } \\
\text { Readings (5) }\end{array}$} & \multirow{3}{*}{\multicolumn{2}{|c|}{$\begin{array}{l}\text { Surface Level } \\
\text { Readings (1) } \\
(-357)\end{array}$}} & \multirow{3}{*}{\multicolumn{2}{|c|}{$\begin{array}{l}\text { LOW Readings } \\
(-357)\end{array}$}} & \multicolumn{2}{|c|}{ Radiation Readings } & \multirow{4}{*}{$\begin{array}{l}\text { Dome } \\
\text { Elevation } \\
\text { Surveys } \\
\text { (OSR/SAR) }\end{array}$} \\
\hline & & & & & & & & Lateral & Drywell & \\
\hline & \multirow{2}{*}{$\begin{array}{c}\text { Watch } \\
\text { List }\end{array}$} & \multirow{2}{*}{$\begin{array}{l}\text { High } \\
\text { Heat }\end{array}$} & & & & & & Readings & Readings & \\
\hline & & & & M.T. & FTC & Gamma & Neutron & & & \\
\hline $101-A$ & $\bar{x}$ & & & & NIA & & & $\mathrm{O} / \mathrm{C}$ & & \\
\hline $102-A$ & & & & 101 & & NT & NA & O/C & & 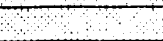 \\
\hline 103-A & & & & $\mathrm{NIA}$ & & & & O/C & & \\
\hline $104-A$ & & $\bar{x}$ & & & NO & $N A$ & N/A & O/C & & \\
\hline $105-A$ & & $\bar{x}$ & & & $\mathrm{NA}$ & NIA & NIA: & O/C & & \\
\hline$\overline{106-A}$ & & & & NA & & $N$ & NA & O/C & & \\
\hline $101-A X$ & $\bar{x}$ & & & $\mathrm{~N} / \mathrm{A}$ & & & & & & \\
\hline 102-AX & & & & & $\mathrm{N} / \mathrm{R}$ & NA & NIA & & & \\
\hline $103-A X$ & $\bar{x}$ & & $\%$ & $\mathrm{NTA}$ & & NIK & N/A & & & \\
\hline $104-A X$ & & & & & NA & $\sqrt{4}$ & $N A$ & & & \\
\hline $101-B$ & & & ৪ & $\mathrm{NA}$ & & $\mathrm{NA}$ & NIA & & & \\
\hline $102-B$ & & & $\%$ & $\mathrm{NA}$ & & $\mathrm{NA}$ & NIA & . & & \\
\hline $103-B$ & $\bar{x}$ & & & NIA & \/\% & NIA & NIA & & & \\
\hline $104-B$ & & & & & $\mathrm{NA}$ & & 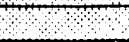 & 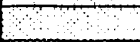 & & \\
\hline $105-8$ & & & & & NIA & & & & & \\
\hline $106-B$ & & & & NIA & & NAA & N/A & & & \\
\hline $107-B$ & & & & & $\mathrm{NA}$ & $\mathrm{NIN}$ & NIA & & & \\
\hline $108-8$ & & & & $\mathrm{NA}$ & & $N A$ & NA & & & \\
\hline $109-B$ & & & & & NIA & NA & NA & & & \\
\hline $110-B$ & & & & & $N$ & NA & NA & & & \\
\hline $111-8$ & & & & NIA & & NIA & N/A & & & \\
\hline $112-B$ & & & & N/A & & NA & NA & & & \\
\hline $201-8$ & & & & & NIA & $\mathrm{NA}$ & NA & & & \\
\hline $202-8$ & & & & & $\mathrm{NH}$ & NUA & NIA & & & $\therefore$ \\
\hline $203-B$ & & & & & $\mathrm{NNA}$ & NIA & N/A & 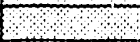 & & \\
\hline $204-B$ & & & & & $\mathrm{NH}$ & $N$ & NA & ४ & ? & 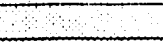 \\
\hline $101-B X$ & & & & & NIA & $\mathrm{N} / \mathrm{A}$ & NIA & & & : \\
\hline $102-B X$ & $\bar{x}$ & & & & $\mathrm{NH}$ & $\mathrm{NA}$ & $\mathrm{NAA}$ & \% & म & \\
\hline $103-B X$ & & & O/C & $\mathrm{NIN}$ & & $\mathrm{NO}$ & NIA & & मे & \\
\hline 104-BX & & & O/S-O/C & $\mathrm{NT}$ & $\mathrm{O} / \mathrm{C}$ & $\mathrm{NOA}$ & NA & 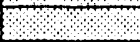 & उ & 8 \\
\hline 105-BX & & & & $\mathrm{NIA}$ & & $\mathrm{N} / \mathrm{A}$ & $N A$ & १ै & & \\
\hline $106-B X$ & $\bar{x}$ & & & $\mathrm{NHA}$ & & $\mathrm{NA}$ & NIA & ४ & प् & , \\
\hline $107-8 X$ & & & $\mathrm{O} / \mathrm{C}$ & NIA & & NIA & NIA & & & \\
\hline
\end{tabular}


TABLE 8. SINGLE-SHELL TANKS MONITORING COMPLIANCE STATUS 149 TANKS (Sheet 2 of 4 )

Information as of $11 / 30 / 92$

\begin{tabular}{|c|c|c|c|c|c|c|c|c|c|c|}
\hline \multirow{4}{*}{$\begin{array}{l}\text { Tank } \\
\text { Number }\end{array}$} & \multirow{2}{*}{\multicolumn{2}{|c|}{ Category }} & \multirow{4}{*}{$\begin{array}{l}\text { Temperature } \\
\text { Readings (5) }\end{array}$} & \multirow{3}{*}{\multicolumn{2}{|c|}{$\begin{array}{c}\text { Surface Level } \\
\text { Readings (1) } \\
\text { (-357) }\end{array}$}} & \multirow{3}{*}{\multicolumn{2}{|c|}{$\begin{array}{l}\text { LOW Readings } \\
(-357)\end{array}$}} & \multicolumn{2}{|c|}{ Radiation Readings } & \multirow{4}{*}{$\begin{array}{l}\text { Dome } \\
\text { Elevation } \\
\text { Surveys } \\
\text { (OSR/SAR) }\end{array}$} \\
\hline & & & & & & & & \multirow{3}{*}{$\begin{array}{c}\text { Lateral } \\
\text { Readings } \\
(-357)\end{array}$} & \multirow{3}{*}{\begin{tabular}{|c|} 
Drywell \\
Readings \\
(OSR/SAR)
\end{tabular}} & \\
\hline & \multirow{2}{*}{$\begin{array}{c}\text { Watch } \\
\text { List }\end{array}$} & \multirow{2}{*}{$\begin{array}{l}\text { High } \\
\text { Heat }\end{array}$} & & & & & & & & \\
\hline & & & & M.T. & FIC & Gamma & Neutron & & & \\
\hline $108-\mathrm{BX}$ & & & O/C & & $8 \%$ & $1 \%$ & $1 \%$ r & ॠN & & \% \\
\hline 109-BX & & & O/C & IN/ & 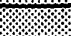 & 1978 & $8 N \mathrm{H}$ & NA & ७ & 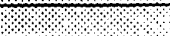 \\
\hline $110-B X$ & $\bar{x}$ & & & & $4 \%$ & 12 & 12 & $\mathrm{NA}$ & ২২ & ए। \\
\hline $111-\mathrm{BX}$ & $\bar{x}$ & & & & NKIS & & & $\mathrm{NA}$ & & \\
\hline $112-B X$ & & & OIC & UT & \% & U. & NA & NA & मे & $1 \%$ \\
\hline 101-BY & $\bar{x}$ & & & & $\mathrm{NI}$ & & & NIA & $\$$ & \%? \\
\hline 102-BY & & & O/S-O/C & & $N A$ & & & NA & $\%$ & \\
\hline 103-BY & $\bar{x}$ & & & & MNA & & & NA & & \\
\hline 104-BY & $\bar{x}$ & & & & $N / \mathrm{N}$ & & \% & $N / A$ & 48 & $\%$ \\
\hline 105-BY & $\bar{x}$ & & & & NI & & & NIA & & म० \\
\hline 106-BY & $\bar{x}$ & & \% & \% & NT & & & NA & 1 & 1 \\
\hline 107-BY & $\bar{x}$ & & & & $\mathrm{Nr}$ & & & $\mathrm{NA}$ & & \\
\hline $108-\overline{B Y}$ & $\bar{x}$ & & & & 14 & 14 & UT & NIA & $1 \%$ & $1 \%$ \\
\hline 109-BY & & & O/S-O/C & NA & $\mathrm{O} / \mathrm{C}$ & & & NIA & & \\
\hline $110-B Y$ & $\bar{x}$ & & \% & & NOT & & 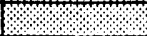 & $N A$ & पे & \\
\hline $111-B Y$ & $\bar{x}$ & & $\sqrt{n}$ & & Nen & ? & $\$$ & N/A & & \\
\hline $112-B Y$ & $\bar{x}$ & & \% & & $\mathrm{N} / \mathrm{A}$ & O/C & & N/A & 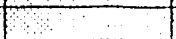 & 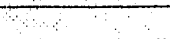 \\
\hline $101-C$ & & & & & NIA & NA & NA & N/A & & \\
\hline $102-C$ & & & & NHA & & N/A & N/A & N/A & N/A & \\
\hline $103-C$ & $\bar{x}$ & & m & NIA & : & NIA & N/A & NA & & \\
\hline $104-C$ & & & & $\mathrm{NT}$ & & $N / 4$ & $\mathrm{NA}$ & N/A & & \\
\hline $105-C$ & & $\bar{x}$ & & NUA & & NA & NAA & NIA & & \\
\hline $106-C(4)$ & $\bar{x}$ & $\bar{x}$ & 8 & $\mathrm{NHA}$ & & $\mathrm{N} / \mathrm{A}$ & NIA & NA & & \\
\hline $107-C$ & & & $\%$ & $\mathrm{NA}$ & & N/A & NIA & NA & & \\
\hline $108-C$ & $\bar{x}$ & & 1 & & $\mathrm{NHA}$ & $\mathrm{NA}$ & $\mathrm{N} / \mathrm{A}$ & N/A & & \\
\hline $109-C$ & $\bar{x}$ & & 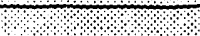 & & NIA & $N / A$ & NA & NIA & & \\
\hline $110-C$ & & & $\%$ & $\%$ & $N A$ & NIA & N/A & N/A & & \\
\hline $111-C$ & $\bar{x}$ & & ও & & $\mathrm{NIA}$ & NIA & NIA & N/A & & \\
\hline $112-C$ & $\bar{x}$ & & \% & & NA & NA & NA & N/A & & \\
\hline $201-C$ & & & $\%$ & & NIA & NA & N/A & N/A & & \\
\hline $202-C$ & & & & & $\mathrm{NA}$ & $\mathrm{NAN}$ & $\mathrm{N} / \mathrm{A}$ & NA & & \\
\hline $203-C$ & & & & & NA & NIA & N/A & NNA & & \\
\hline $204-C$ & & & OIC & & NIA & NA & $\mathrm{NA}$ & N/A & & \\
\hline $101-5$ & & & & NA & & & & N/A & & \\
\hline $102-5$ & $\bar{x}$ & & 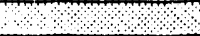 & NA & & & & N/A & & \\
\hline $103-5$ & & & एक & NIA & & & & NMA & & \\
\hline $104-5$ & & & & & NA & $\mathrm{NAS}$ & $\mathrm{NA}$ & N/A & & \\
\hline $105-5$ & & & & N/A & & & & N/A & $\cdots$ & \\
\hline $106-5$ & & & & NHA & & & & NA & 18 & \\
\hline $107-5$ & & & & $\mathrm{NIA}$ & & NIA & NIA & NA & & \\
\hline $108-5$ & & & & & & & & $\mathrm{~N} / \mathrm{A}$ & & \\
\hline $109-5$ & & & & NA & & & & NIA & & \\
\hline $110-5$ & & & & N/N & & & & NA & 20 & मे \\
\hline $111-S$ & $\bar{x}$ & & & $\mathrm{NA}$ & & & & N/A & & 6 \\
\hline $112-\mathrm{S}$ & $\bar{x}$ & & & $\mathrm{~N}$ & & & & NIA & & \\
\hline $101-5 x$ & $x$ & & & $\mathrm{NA}$ & & & & NIA & & \\
\hline $102-5 X$ & $\bar{x}$ & & & $\mathrm{NA}$ & & & & NA & & $\because$ \\
\hline $103-5 x$ & $\bar{x}$ & & & NA & $\mathrm{O} / \mathrm{C}$ & & & N/A & & \\
\hline $104-5 X$ & $\mathrm{x}$ & & & $\mathrm{NAA}$ & & O/S-O/C & O/S-O/C & N/A & & \\
\hline $105-5 x$ & $\bar{x}$ & & & NA & & 7 & 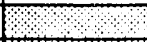 & $\mathrm{O} / \mathrm{C}$ & & \\
\hline $106-5 x$ & $\bar{x}$ & & & NA & & & & NA & & \\
\hline $107-5 X$ & & $\bar{x}$ & & & NA & NIA & NIA & O/C & & \\
\hline $108-5 X$ & & $x$ & & & $\mathrm{NAA}$ & $N A$ & N/A & $O / C$ & & \\
\hline
\end{tabular}


TABLE 8. SINGLE-SHELL TANKS MONITORING COMPLIANCE STATUS 149 TANKS (Sheet 3 of 4 )

Information as of $11 / 30 / 92$

\begin{tabular}{|c|c|c|c|c|c|c|c|c|c|c|}
\hline \multirow{4}{*}{$\begin{array}{l}\text { Tank } \\
\text { Number }\end{array}$} & \multirow{2}{*}{\multicolumn{2}{|c|}{ Category }} & \multirow{4}{*}{$\begin{array}{l}\text { Temperature } \\
\text { Readings (5) }\end{array}$} & \multirow{3}{*}{\multicolumn{2}{|c|}{$\begin{array}{c}\text { Surface Level } \\
\text { Readings (1) } \\
(-357)\end{array}$}} & \multirow{3}{*}{\multicolumn{2}{|c|}{$\begin{array}{c}\text { LOW Readings } \\
(-357)\end{array}$}} & \multicolumn{2}{|c|}{ Radiation Readings } & \multirow{4}{*}{$\begin{array}{c}\text { Dome } \\
\text { Elevation } \\
\text { Surveys } \\
\text { (OSR/SAR) }\end{array}$} \\
\hline & & & & & & & & \multirow{3}{*}{\begin{tabular}{|c|} 
Lateral \\
Readings \\
$(-357)$ \\
\end{tabular}} & \multirow{3}{*}{\begin{tabular}{|c|} 
Drywell \\
Readings \\
(OSR/SAR)
\end{tabular}} & \\
\hline & \multirow{2}{*}{$\begin{array}{c}\text { Watch } \\
\text { List }\end{array}$} & \multirow{2}{*}{$\begin{array}{l}\text { High } \\
\text { Heat }\end{array}$} & & & & & & & & \\
\hline & & & & M.T. & FIC & Gamma & Neutron & & & \\
\hline $102-5 \times(4)$ & $\bar{x}$ & $\bar{x}$ & & & 1Y. & $8 \mathrm{NTW}$ & WNo & OIC & & \\
\hline $110-5 X$ & & $\bar{x}$ & & & N. & Nom & $N A$ & O/C & & \\
\hline $111-5 x$ & & $\bar{x}$ & & & 14 & $8 \%$ & 14 & O/C & & \\
\hline $112-5 x$ & & $\bar{x}$ & & & 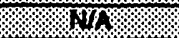 & 26 & NA & O/C & & \\
\hline $113-5 x$ & & & & & $\mathrm{NH}$ & 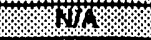 & NI & NA & & \\
\hline $114-5 x$ & & $\bar{x}$ & & & 102 & MIN & $\mathrm{NA}$ & $\mathrm{O} / \mathrm{C}$ & & \\
\hline $115-5 x$ & & & O/S - O/C & & N1. & $4 \%$ & $4+4$ & O/C & & \\
\hline 101-T & $\bar{x}$ & & & N19 & & $N 2$ & NTH & NA & & \\
\hline 102-T & & & OIS - O/C & 40 & & 10 & 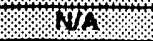 & $\mathrm{NHA}$ & & 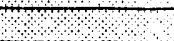 \\
\hline 103-T & & & & $\mathrm{N} / \mathrm{A}$ & & $\mathrm{NI}$ & $N A$ & NIA & & \\
\hline $104-T$ & & & & & $\mathrm{NT}$ & & & NA & $\because$ & $8 \alpha$ \\
\hline 105-T & & & $O / S-O / C$ & $\mathrm{NHA}$ & & $\mathrm{N} / \mathrm{N}$ & NA & NIA & & \\
\hline $106-T$ & & & & $0 \mathrm{H}$ & $m$ & $\mathrm{HA}$ & NA & N/A & + & \\
\hline $107-T$ & $x$ & & & NIA & & $N \mathrm{NA}$ & NIA & NIA & 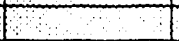 & \\
\hline $108-T$ & & & OIC & & NA & $\mathrm{NIA}$ & NA & $\mathrm{NA}$ & 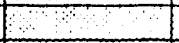 & $\therefore \quad \vdots$ \\
\hline $109-T$ & & & $O / C$ & $\mathrm{NIA}$ & & NIA & NIA & NIA & & \\
\hline $110-T$ & $x$ & & & $\mathrm{NA}$ & 8 & & +1 & NIA & & $\because$ \\
\hline $111-T$ & & & & NA & & & & NIA & . & $\therefore$ \\
\hline $112-T$ & & & & NIA & & NA & NAA & NA & $\cdots$ & $\therefore$ \\
\hline $201-T$ & & & & & NA & NIA & NIA & NA & 0 & \\
\hline $202-T$ & & & & & $\mathrm{NHA}$ & $\mathrm{NA}$ & NA & NIA & & n \\
\hline 203-T & & & & & $\mathrm{NA}$ & NIA & $\mathrm{N}$ & NIA & & \\
\hline 204-T & & & & & $\mathrm{NH}$ & $N+2$ & NAA & NA & & 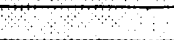 \\
\hline $101-\mathrm{TX}$ & & & $O / S-O / C$ & $\mathrm{~N} / \mathrm{A}$ & & $\mathrm{N} / \mathrm{N}$ & NIA & NIA & 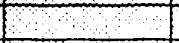 & \\
\hline $102-\mathrm{TX}$ & & & $\mathrm{O} / \mathrm{C}$ & & NA & & & NIA & & \\
\hline 103-TX & & & O/C & NIA & & NIA & NIA & N/A & 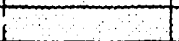 & \\
\hline $104-\mathrm{TX}$ & & & O/C & $\mathrm{NA}$ & & WNA & NIA & NA & 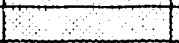 & \\
\hline 105-TX & $x$ & & & & NA & O/S-O/C & O/S-O/C & NIA & & \\
\hline 106-TX & & & OIC & & NNA & & & N/A & . & \\
\hline $107-T X$ & & & O/C & NIA & & NA & NA & NIA & & मु_ \\
\hline 108-TX & & & O/C & $\mathrm{NH}$ & & & & $\mathrm{NA}$ & & \\
\hline $109-\mathrm{TX}$ & & & $\mathrm{O} / \mathrm{C}$ & NA & & & & N/A & & \\
\hline $110-T X$ & & & O/C & & $N \mathrm{NA}$ & & & NIA & 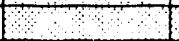 & $1+$ \\
\hline $111-T X$ & & & $\mathrm{O} / \mathrm{C}$ & & $\mathrm{NA}$ & & & NIA & & \\
\hline $112-\mathrm{TX}$ & & & $\mathrm{O} / \mathrm{C}$ & & $N \mathrm{~N}$ & & & NA & & \\
\hline $113-\mathrm{TX}$ & & & $\mathrm{O} / \mathrm{C}$ & & $\mathrm{NA}$ & & & $N / A$ & & 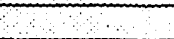 \\
\hline 114-TX & & & O/C & 3 & NA & & & NIA & & \\
\hline $115-T X$ & & & $\mathrm{O} / \mathrm{C}$ & & NA & & & NIA & & \\
\hline $116-\mathrm{TX}$ & & & $\mathrm{O} / \mathrm{S}-\mathrm{O} / \mathrm{C}$ & & $N A$ & NHA & NIA & $N / A$ & & \\
\hline $117-\mathrm{TX}$ & & & $O / C$ & & NIA & O/C & & $N / A$ & & \\
\hline $118-T X$ & $\bar{x}$ & & & NA & & O/C & & NIA & & \\
\hline $101-T Y$ & $\bar{x}$ & & & NAA & & $\mathrm{NA}$ & N/A & N/A & & \\
\hline $102-T Y$ & & & & $\mathrm{~N}$ & & NA & N/A & NA & & \\
\hline 103-TY & $x$ & & & NIA & & $\mathrm{O} / \mathrm{C}$ & & N/A & 3 & \\
\hline 104-TY & $x$ & & & N/S & & $\mathrm{NA}$ & $N \mathbf{N A}$ & NIA & & \\
\hline 105-TY & & & & & NIA & $\mathrm{NA}$ & $\mathrm{N}$ & NIA & & \\
\hline 106-TY & & & & & $\mathrm{NA}$ & NA & NIA & NIA & & \\
\hline $101-U$ & & & & & NA & NA & NIA & NIA & & \\
\hline $102--U$ & & & & $N \mathrm{H}$ & & & & NA & & \\
\hline $103-U$ & $x$ & & & $\mathrm{NA}$ & & & & NIA & & \\
\hline $104-U$ & & & O/S-O/C & & WA & $\mathrm{NHA}$ & $\mathrm{NIA}$ & NA & & \\
\hline $105-U$ & $\bar{x}$ & & & $\mathrm{NA}$ & & & & NA & & \\
\hline $106-U$ & $x$ & & & $\mathrm{NAS}$ & & & & $\mathrm{N} / \mathrm{A}$ & & \\
\hline
\end{tabular}




\section{TABLE 8. SINGLE-SHELL TANKS MONITORING COMPLIANCE STATUS 149 TANKS (Sheet 4 of 4 )}

Information as of $11 / 30 / 92$

\begin{tabular}{|c|c|c|c|c|c|c|c|c|c|c|}
\hline \multirow{4}{*}{$\begin{array}{l}\text { Tank } \\
\text { Number }\end{array}$} & \multirow{2}{*}{\multicolumn{2}{|c|}{ Category }} & \multirow{4}{*}{$\begin{array}{l}\text { Temperature } \\
\text { Readings (5) }\end{array}$} & \multirow{3}{*}{\multicolumn{2}{|c|}{$\begin{array}{c}\text { Surface Leve! } \\
\text { Readings (1) } \\
\text { (-357) }\end{array}$}} & \multirow{3}{*}{\multicolumn{2}{|c|}{$\begin{array}{l}\text { LOW Readings } \\
\text { (-357) }\end{array}$}} & \multicolumn{2}{|c|}{ Radiation Readings } & \multirow{4}{*}{$\begin{array}{c}\text { Dome } \\
\text { Elevation } \\
\text { Surveys } \\
\text { (OSR/SAR) } \\
\end{array}$} \\
\hline & & & & & & & & \multirow{3}{*}{$\begin{array}{c}\text { Lateral } \\
\text { Readings } \\
(-357)\end{array}$} & \multirow{3}{*}{\begin{tabular}{|c|} 
Drywell \\
Readings \\
(OSR/SAR)
\end{tabular}} & \\
\hline & \multirow{2}{*}{$\begin{array}{c}\text { Watch } \\
\text { List }\end{array}$} & \multirow{2}{*}{$\begin{array}{l}\text { High } \\
\text { Heat }\end{array}$} & & & & & & & & \\
\hline & & & & M.T. & FIC & Gamma & Neutron & & & \\
\hline $107-U$ & $x$ & & & Nor & $0 / 5$ & & & १NA & & \\
\hline $108-U$ & $\bar{x}$ & & & 14 & & & & $\mathrm{NIA}$ & & \\
\hline $109-U$ & $\bar{x}$ & & & 1078 & & & & NIF & & \\
\hline $110-U$ & & & & 43 & & \$N & NY & NA & & \\
\hline $111-U$ & & & & NIS & & & & NA & & 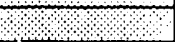 \\
\hline $112-U$ & & & & & $\mathrm{~N}+\mathrm{A}$ & $\mathrm{NO}$ & NA & $N A$ & & \\
\hline 201-U & & & & & No & NA & WA & $\sqrt{4}$ & & \\
\hline $202-U$ & & & & & NTH & $\mathrm{NH}$ & N/A & NH & 4 & \\
\hline $203-U$ & & & $O / C$ & & $\mathrm{NA}$ & NII & NIS & NA & 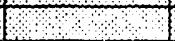 & म \\
\hline 204-U & & & O/C & & $\mathrm{N} / \mathrm{A}$ & N/A & NA & $\mathrm{N} / \mathrm{A}$ & 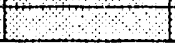 & \\
\hline \multicolumn{11}{|c|}{ Catch Tanks } \\
\hline$A-302-A$ & N/A & N/A & $\mathrm{Nu}$ & $\mathrm{NK}$ & & NA & $\mathrm{NAA}$ & $\mathrm{N} / \mathrm{A}$ & NIA & N/A \\
\hline$A-302-B$ & N/A & N/A & NAA & & NIA & NA & NA & NIA & N/A & N/A \\
\hline 311-ER & N/A & N/A & $\mathrm{N} / \mathrm{A}$ & N/A & & NA: & N/A & NA & N/A & N/A \\
\hline Vent Sta. & N/A & N/A & NIA & $\mathrm{O} / \mathrm{C}$ & NA & N/A & NIA & N/A & N/A & N/A \\
\hline $151-A Z$ & N/A & N/A & NA & $\mathrm{NA}$ & & NA & N/A & N/A & N/A & N/A \\
\hline $154-A Z$ & N/A & N/A & NA & & NA & NA & NA & N/A & N/A & N/A \\
\hline$S-302$ & N/A & N/A & NIA & NA & & N/A & N/A & NA & N/A & N/A \\
\hline$S-302-A$ & N/A & N/A & NA & $N A$ & O/C & N/A & $N A$ & N/A & N/A & N/A \\
\hline $\mathrm{TX}-302-\mathrm{B}$ & N/A & N/A & NA & $\mathrm{O} / \mathrm{C}$ & N/A & NA & N/A & N/A & NA & N/A \\
\hline$T X-302-C$ & N/A & N/A & NA & $\mathrm{N} / \mathrm{A}$ & & NA & NA & NIA & N/A & N/A \\
\hline U-301-B & N/A & N/A & $\mathrm{NI}$ & $\mathrm{NHA}$ & & NHA & NA & $N A$ & N/A & $\mathrm{N} / \mathrm{A}$ \\
\hline$U X-302-A$ & N/A & N/A & NA & NA & & $N /$ & NA & N/A & N/A & N/A \\
\hline $141-S$ & N/A & N/A & NA & & N/A & NA & NA & N/A & $\mathrm{N} / \mathrm{A}$ & N/A \\
\hline $142-S$ & N/A & N/A & $N / A$ & & NA & $N A$ & NA & $\mathrm{N} / \mathrm{A}$ & N/A & N/A \\
\hline \multirow[t]{2}{*}{$\begin{array}{l}\text { Totals: } \\
149 \text { tanks }\end{array}$} & \multirow{2}{*}{$\begin{array}{c}48 \\
\text { Watch } \\
\text { List } \\
\text { Tanks } \\
\text { (4) }\end{array}$} & \multirow{2}{*}{$\begin{array}{c}11 \\
\text { High } \\
\text { Heat } \\
\text { Tanks } \\
\text { (4) }\end{array}$} & \multirow{2}{*}{$\begin{array}{l}\text { O/C: } \\
33 \text { tank8 - } \\
\text { (semiannual } \\
\text { monitoring } \\
\text { frequency) (5) }\end{array}$} & \multirow[t]{2}{*}{$\begin{array}{l}\mathrm{O} / \mathrm{C}: \\
2 \text { tank8 }\end{array}$} & \multirow[t]{2}{*}{$\begin{array}{l}\text { O/C: } \\
4 \text { tanks }\end{array}$} & $\begin{array}{l}\text { O/C: } \\
6 \text { tank8 }\end{array}$ & $\begin{array}{l}O / C \text {. } \\
2 \text { tanks }\end{array}$ & \multirow[t]{2}{*}{$\begin{array}{l}\text { O/C: } \\
15 \text { tanks }\end{array}$} & \multirow[t]{2}{*}{0} & \multirow[t]{2}{*}{0} \\
\hline & & & & & & \multicolumn{2}{|c|}{$\begin{array}{l}58 \text { tanks have LOWs } \\
\text { (2 are O/S) }\end{array}$} & & & \\
\hline
\end{tabular}

(1) All SSTs have either manual tape or FIC, with the exception of 108-S which has both. All SST FICs are connected to CASS, (with the exception of 106-BX); however, the connection for many tanks is broken. For such cases, manual field readings are taken. Manual surface level readings include readings taken by manual tape, manual FIC (not connected to Computer Automated Surveillance System, 106-BX), manual readings of automatic FIC (if CASS is printing " 0 "), or automatic FIC. In some cases, the surface level readings are taken using a zip cord. While less accurate, such readings are acceptable for meeting the surface level reading requirements.

(2) Psychrometric readings are taken on tanks with active exhausters (High heat tanks 104/105-A, 105/106-C, 107, 108, 109, 110, 111, 112, and 114-SX). Psychrometrics were not taken from 9/91 to 9/92. Exhauster down on 104/105-A since 10/1/91. 105/106-C and SX tanks have resumed psychrometric readings on a monthly frequency. Psychrometric trequency not specified in -357 or operating procedures.

(3) In-tank Photographs are not required by -357. Last in-tank photographs in SSTs were taken September 1990.

(4) Two tanks are on both category lists (106-C, 109-SX).

(5) Temperature readings may be regulated by OSD, -357 , or POP. Additionally, high heat load tanks are regulated by OSR/SAR. Thermcouples in the 9 tanks designated O/S-O/C are out of service; there are either no thermocouple probes in these tanks, or probes have been cut off, covered over, or are otherwise not functioning. The OSD does not require readings or repair of out-of-service thermocouples in non-heat load $(>40,000 \mathrm{Btu} / \mathrm{hr})$ tanks.

BM Hanlon, 12/23/92 
WHC-EP-0182-56

\section{TABLE 9. DOUBLE-SHELL TANKS MONITORING COMPLIANCE STATUS 28 TANKS}

The following table indicates whether Double-Shell tank monitoring is in compliance with the requirements as specified in the applicable documents:

NOTE:

All DST tank temperature tree monitoring is in compliance.

Dome Elevation Surveys are not required for DSTs.

Psychrometrics (2)

In-tank Photographs (3)

\begin{tabular}{|c|c|}
\hline LEGEND: & \\
\hline $10.0 \%$ & $=$ In compliance with all applicable documentation \\
\hline ON & $=$ Out of compliance with docurnentation \\
\hline-357 & $\begin{aligned}= & \text { WHC-SD-WM-T1-357. "Waste Storage Tank Status and Leak } \\
& \text { Detection Criteria" }\end{aligned}$ \\
\hline M.T. & $=$ Manual Tape \\
\hline & = Food Inetrument Company \\
\hline OSPSAR & $\begin{aligned}= & \text { Operatione Safoty Requirements/Safety Analysis Report } \\
& \text { Requirement8 }\end{aligned}$ \\
\hline N/A & $=$ Not Applicable (i.e., no M.T., FIC installed) \\
\hline O/S & $=$ Out of Service \\
\hline W.F. & $=$ Weight Factor \\
\hline Rad. & = Radiation \\
\hline
\end{tabular}

Information as of $11 / 30 / 92$

\begin{tabular}{|c|c|c|c|c|c|c|c|}
\hline \multirow{3}{*}{$\begin{array}{l}\text { Tank } \\
\text { Number }\end{array}$} & \multirow[b]{3}{*}{ Watch List } & \multirow{3}{*}{$\begin{array}{c}\text { Temperature } \\
\text { Readings } \\
(4) \\
\text { (OSR/SAR) } \\
\end{array}$} & \multirow{2}{*}{\multicolumn{2}{|c|}{$\begin{array}{l}\text { Surface Level } \\
\text { Readings (1) } \\
\text { (-357, OSR/SAR) }\end{array}$}} & \multicolumn{3}{|c|}{ Radiation Readings } \\
\hline & & & & & \multicolumn{2}{|c|}{$\begin{array}{c}\text { Leak Detection } \\
\text { Pits (5) } \\
\text { (-357, OSR/SAR) }\end{array}$} & \multirow{2}{*}{$\begin{array}{c}\text { Annulus } \\
(-357)\end{array}$} \\
\hline & & & M.T. & FIC & W.F. & Had. & \\
\hline 101-AN & & & Nots & & ২ & & \\
\hline 102-AN & & & N/A & & & & \\
\hline 103-AN & $\bar{x}$ & & $\mathrm{~N} / \mathrm{A}$ & & & & \\
\hline 104-AN & $\bar{x}$ & & NIA & & & O/C & \\
\hline $105-A N$ & $x$ & & NA & & & & \\
\hline 106-AN & & & NA. & & & & \\
\hline 107-AN & & & NA & & & & \\
\hline \multicolumn{8}{|l|}{ 101-AP } \\
\hline \multicolumn{8}{|l|}{ 102-AP } \\
\hline \multicolumn{8}{|l|}{ 103-AP } \\
\hline \multicolumn{8}{|l|}{ 104-AP } \\
\hline \multicolumn{8}{|l|}{ 105-AP } \\
\hline \multicolumn{8}{|l|}{ 106-AP } \\
\hline \multicolumn{8}{|l|}{ 107-AP } \\
\hline \multicolumn{8}{|l|}{ 108-AP } \\
\hline 101-AW & $x$ & & ( & O/S & $\lcm{.1 .1}$ & O/C & \\
\hline \multicolumn{8}{|l|}{ 102-AW } \\
\hline 103-AW & & & & $0 / 5$ & & & \\
\hline \multicolumn{8}{|l|}{ 104-AW } \\
\hline 105-AW & & & 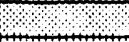 & O/S & & & \\
\hline \multicolumn{8}{|l|}{ 106-AW } \\
\hline 101-AY & & & & O/S & & & $\mathrm{O} / \mathrm{C}$ \\
\hline 102-AY & & & $\lcm{1}$ & 10 & 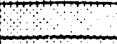 & 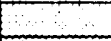 & $\mathrm{O} / \mathrm{C}$ \\
\hline $101-A Z$ & & & & O/S & & 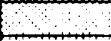 & $\mathrm{O} / \mathrm{C}$ \\
\hline 102-AZ & & & 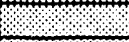 & O/S & 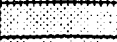 & & $\mathrm{O} / \mathrm{C}$ \\
\hline 101-SY & $x$ & & 1,1, & & 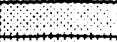 & $\mathrm{O} / \mathrm{C}$ & \\
\hline 102-SY & & & $\%$ & O/S & $\$$ & $\mathrm{O} / \mathrm{C}$ & \\
\hline 103-SY & $x$ & & & 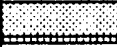 & 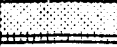 & $\mathrm{O} / \mathrm{C}$ & \\
\hline $\begin{array}{l}\text { Totals: } \\
28 \text { tank8 }\end{array}$ & $\begin{array}{c}6 \\
\text { Watch List Tanks } \\
\end{array}$ & $\begin{array}{l}\text { O/C: } \\
0 \text { (4) }\end{array}$ & $\begin{array}{l}\text { O/C: } \\
0\end{array}$ & $\begin{array}{l}\text { O/C: } \\
0\end{array}$ & $\begin{array}{l}\text { O/C: } \\
0\end{array}$ & $\begin{array}{l}\text { O/C: } \\
5 \text { tanks }\end{array}$ & $\begin{array}{l}\text { O/C: } \\
4 \text { lanks }\end{array}$ \\
\hline
\end{tabular}

(1) All DSTs have both manual tape and FIC, with the exception of the AN Tank Farm which only has FICs. The manual tape is used when the FIC is out of service. O/C will be shown when no readings are obtained.

(2) Psychrometric readings are taken on DSTs. All DSTs are equipped with active exhausters. Psychrometrics were not taken from 9/91 to 9/92; readings on some tanks were resumed beginning October 1992. Frequency of psychrometric monitoring is not specified in -357 or operating procedures.

(3) In-tank photographs are not required by $\mathbf{- 3 5 7}$. Last in-tank photographs in DSTs were taken April 1989.

(4) DST temperature tree readings are taken weekly, with the exception of 101-SY which are obtained shiftwise with increased readings taken prior to and following gas venting.

(5) Failure of both leak detection systems requires repair of at least one system within 5 working days. Failure of one system only, repair must be within 10 working days. Per -357 . If repair of out-of-service systems exceeds these timeframes, systems are O/C. Out-of-service systems which have not exceeded these timeframes will be shown as O/S. 


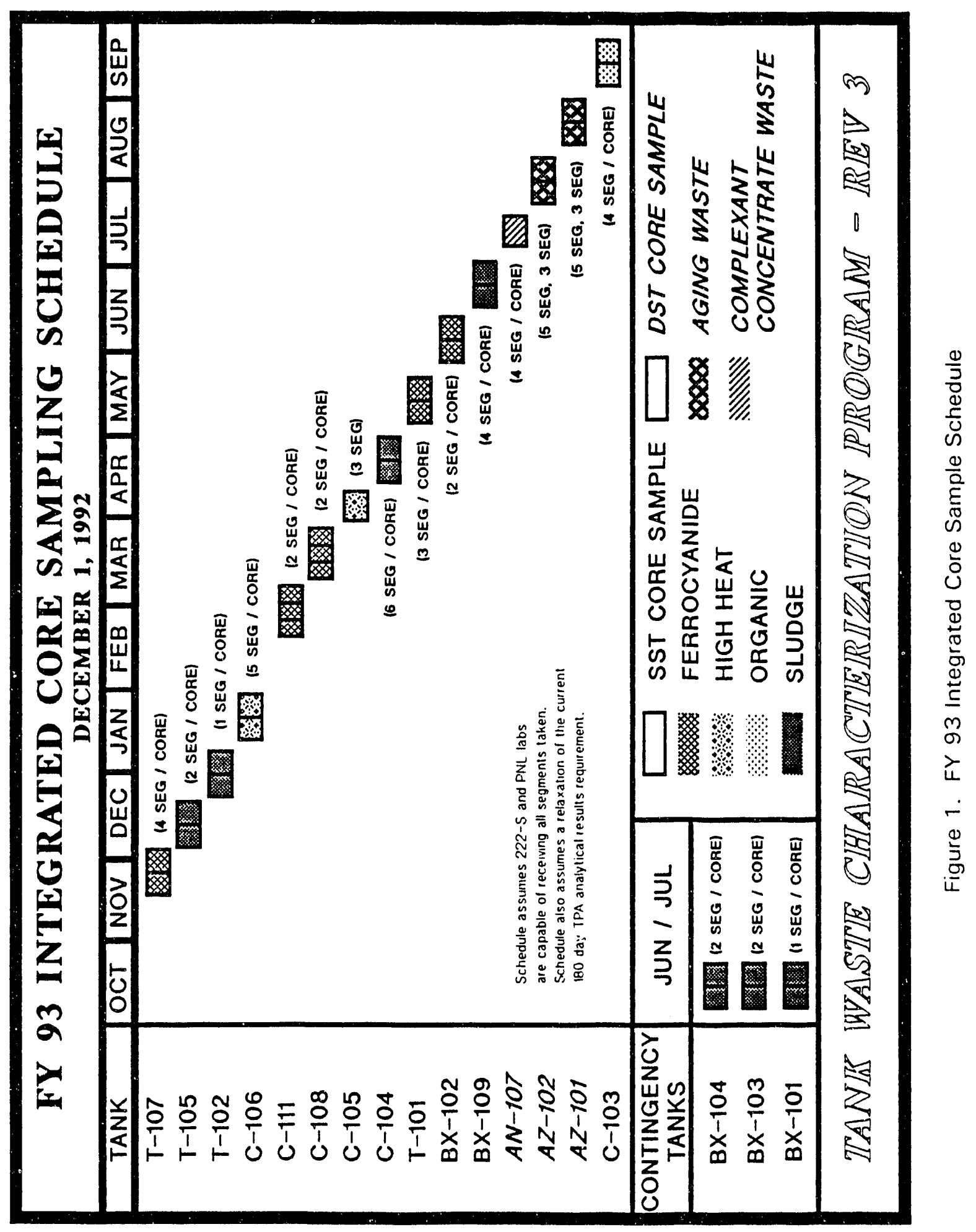




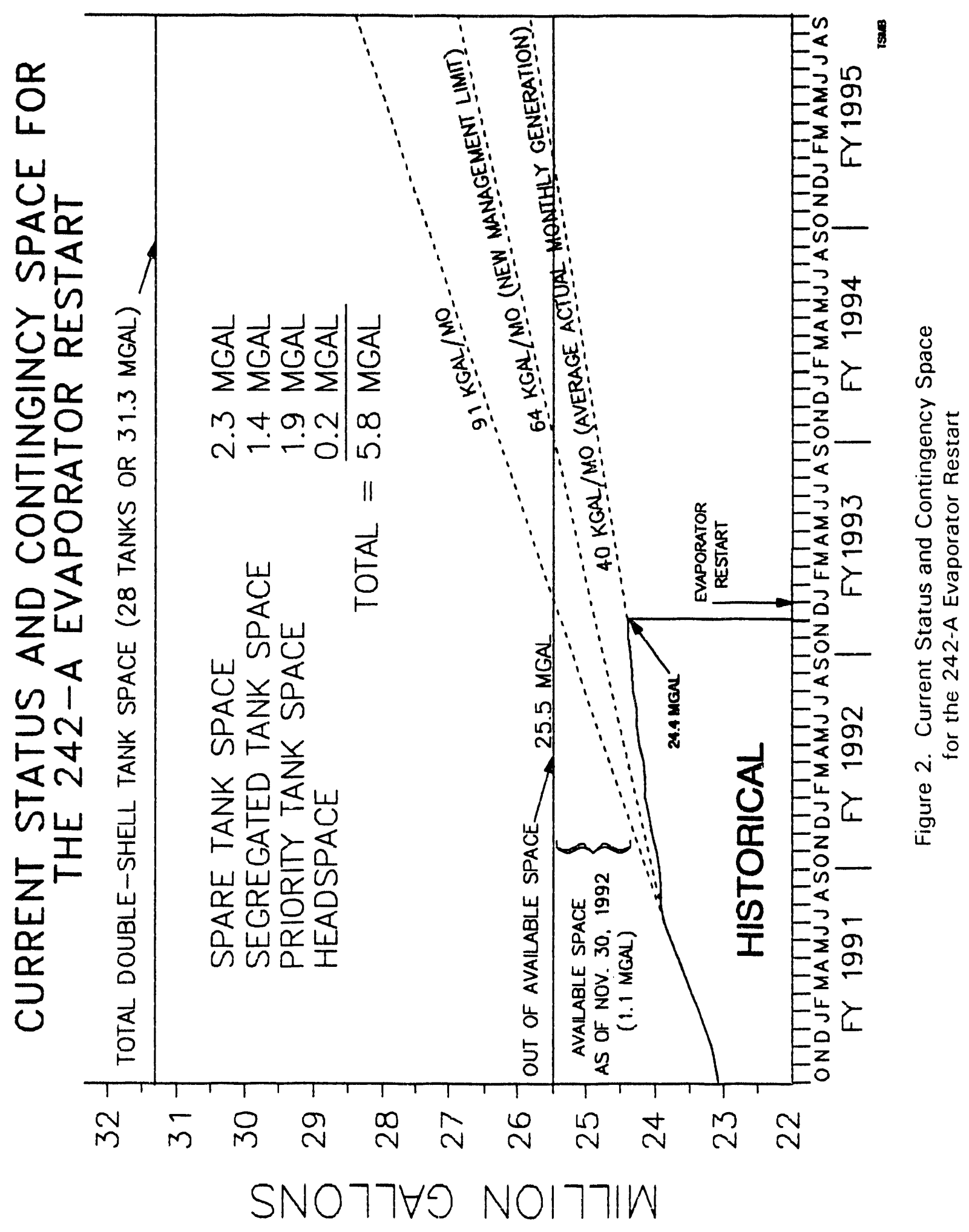


WHC-EP-0182-56

\section{APPENDIX A}

\section{TANK AND EQUIPMENT CODE} AND STATUS DEFINITIONS 


\section{WHC-EP-0182-56}

This page intentionally left blank 


\section{TANK AND EQUIPMENT CODE/STATUS DEFINITIONS}

November 1992

\section{TANK STATUS CODES}

\section{WASTE TYPE}

$\begin{array}{ll}\text { AGING } & \text { Aging Waste (Neutralized Current Acid Waste [NCAW]) } \\ \text { CC } & \text { Complexant Concentrate Waste } \\ \text { CP } & \text { Concentrated Phosphate Waste } \\ \text { DC } & \text { Dilute Complexed Waste } \\ \text { DN } & \text { Dilute Non-Complexed Waste } \\ \text { DSS } & \text { Double-Shell Slurry } \\ \text { DSSF } & \text { Double-Shell Slurry Feed } \\ \text { NCPLX } & \text { Non-Complexed Waste } \\ \text { PD/PN } & \text { Plutonium-Uranium Extraction (PUREX) Neutralized Cladding } \\ & \text { Removal Waste (NCRW) transuranic Waste (TRU) } \\ \text { PT } & \text { Plutonium Finishing Plant (PFP) TRU Solids }\end{array}$

TANK USE (DOUBLE-SHELL TANKS ONLY)

CWHT Concentrated Waste Holding Tank

DRCVR Dilute Receiver Tank

EVFD Evaporate Feed Tank

GRTFD Grout Feed Tank

SRCVR Slurry Receiver Tank

\section{SOLID AND LIQUID VOLUME DETERMINATION METHODS}
F
$M$
P
Food Instrument Company (FIC) Automatic Surface Level Gauge Manual Tape Surface Level Gauge
Photo Evaluation
S Sludge Level Measurement Device

\section{DEFINITIONS}

\section{WASTE TYPES}

Aging Waste (AGING)

Concentrated Complexant (CC)

Concentrated Phosphate Waste $(C P)$

Dilute Complexed Waste (DC)
High level, first cycle solvent extraction waste from the PUREX plant (NCAW)

Concentrated product from the evaporation of dilute complexed waste.

Waste originating from the decontamination of $100 \mathrm{~N}$ Area reactor. Concentration of this waste produces concentrated phosphate waste.

Characterized by a high content of organic carbon including organic complexants:

ethylenediaminetetra-acetic acid (EDTA), citric acid, hydroxyethyl-ethylenediaminetriacetic acid (HEDTA), and iminodiacetate (IDA) being the major complexants used. Main sources of DC waste in the DST system are saltwell liquid inventory. 
Dilute Non-Complexed Waste (DN)

Low activity liquid waste originating from $T$ and $S$ Plants, the 300 and 400 Areas, PUREX facility (decladding supernatant and miscellaneous wastes), $100 \mathrm{~N}$ Area (sulfate waste), B Plant, saltwells, and PFP (supernate).

Double-She11

Slurry (DSS)

Double-Shell

Slurry Feed (DSSF)

Non-complexed (NCPLX)

PUREX Decladding $(\mathrm{PD} / \mathrm{PN})$

PFP TRU Solids (PT)

Drainable Interstitial Liquid (DIL)

Supernate

Ferrocyanide

\section{WASTE STATUS}

In-Service Tank

Out-of-Service Tank
Waste that exceeds the sodium aluminate saturation boundary in the evaporator without exceeding receiver tank composition limits. For reporting purposes, DSS is considered a solid.

Waste concentrated just before reaching the sodium aluminate saturation boundary in the evaporator without exceeding receiver tank composition limits. This form is not as concentrated as DSS.

General waste term applied to all Hanford site liquors not identified as complexed.

PUREX Neutralized Cladding Removal Waste (NCRW) is the solids portion of the PUREX plant neutralized cladding removal waste stream; received in Tank Farms as a slurry. NCRW solids are classified as transuranic (TRU) waste.

TRU solids fraction from PFP Plant operations.

Interstitial liquid that is not held in place by capillary forces, and will therefore migrate or move by gravity.

The liquid above the solids in waste storage tanks.

A compound of iron and cyanide commonly expressed as FeCN. The actual formula for the ferrocyanide anion is $\left[\mathrm{Fe}(\mathrm{CN})_{6}\right]^{-4}$.

The waste classification of a tank being used, or planned for use, for the storage of liquid (in excess of a minimum supernatant liquid heel) in conjunction with production and/or waste processing.

A tank which does not meet the definition of an inservice tank. Before September 1988, these tanks were defined as inactive in this report. [Note: Al1 single-shell tanks (SST) are out of service.]

\section{STABILIZATION (Single-Shell Tanks only)}

Interim Stabilized

(IS)
A tank which contains less than 50,000 gal of drainable interstitial liquid and less than 5,000 gal of supernatant liquid. If the tank was 
jet pumped to achieve interim stabilization, then the jet pump flow must also have been at or below $0.05 \mathrm{gpm}$ before interim stabilization criteria is met.

\section{ISOLATION (Single-Shell Tanks only)}

\author{
Partially \\ Interim Isolated \\ (PI)
}

Interim :solated (II)

\section{TANK INTEGRITY}

Sound

Assumed Ltaker
The administrative designation reflecting the completion of the physical effort required for Interim Isolation except for isolation of risers and piping that is required for jet pumping or for other methods of stabilization.

The administrative designation reflecting the completion of the physical effort required to minimize the addition of liquids into an inactive storage tank, process vault, sump, catch tank, or diversion box.

The integrity classification of a waste storage tank for which surveillance data indicate no loss of liquid attributed to a breach of integrity.

The integrity classification of a waste storage tank for which surveillance data indicate a loss of 1 iquid attributed to a breach of integrity.

\section{TANK INVESTIGATION}

Intrusion

A term used to describe the infiltration of liquid into a waste tank.

\section{SURVEILLANCE INSTRUMENTATION}

Drywells

Drywells are vertical boreholes with 6 -in. (internal diameter) carbon steel casings positioned radially around SSTs. Periodic monitoring is done by gamma radiation or neutron sensors to obtain scan profiles of radiation or moisture in the soil as a function of well depth, which could be indicative of tank leakage. These wells range between 50 and $250 \mathrm{ft}$ in depth, and are monitored between the range of 50 to $150 \mathrm{ft}$. The wells are sealed when not in use. They are called drywells because they do not penetrate to the water taole and are therefore usually "dry." The drywell frequency monitoring schedule calls for 105 drywells weekly, 91 biweekly, 41 monthly, 151 quarterly, and 371 annually.

Laterals

Laterals are horizontal drywells positioned under single-shell waste storage tanks to detect radionuclides in the soil which could be indicative of tank leakage. These drywells are monitored by radiation detection probes. Laterals are 4-in. 
Surface Levels

Automatic FIC

Annulus

Liquid Observa-

tion Well (LOW)

Thermocouple (TC) inside diameter steel pipes located 8 to $10 \mathrm{ft}$ below the tank's concrete base. There are three laterals per tank. Laterals are located only in A and SX farms.

The surface level measurements in all waste storage tanks are monitored by manual or automatic conductivity probes, and recorded and transmitted or inputted to the Computer Automated Surveillance System (CASS).

An automatic waste surface level measurement device is manufactured by the Food Instrument Company (FIC). The instrument consists of a conductivity electrode (plummet) connected to a calibrated steel tape, a steel tape reel housing and a controller that automatically raises and lowers the plummet to obtain a waste surface level reading. The controller can provide a digital display of the data and also transmit the reading to the CASS. Some tanks have gauges connected to CASS and others are read manualiy.

The annulus is the space between the inner and outer shells on DSTs. Drain channels in the insulating and/or supporting concrete carry any leakage to the annulus space where conductivity probes are installed. Alarms from the annunciators are received by CASS. Continuous Air Monitoring (CAM) alarms are also located in the annulus. The annulus conductivity probes and radiation detectors are the primary means of leak detection for all DSTs.

In-tank liquid observation wells are used for monitoring the interstitial liquid level (ILL) in single-shell waste storage tanks. The wells are constructed of fiberglass, tefzel-reinforced epoxypolyester resin, sized to extend to within 1 in. of the bottom of the tank steel liner. They are sealed at their bottom ends and have a nominal outside diameter of 3.5 in. Three probes are used to monitor changes in the ILL: acoustic; gamma; and neutron, which can indicate intrusions or leakage by increases or decreases in the ILL. There are 58 LOWs (57 are in operation) installed in SSTs that contain or are capable of containing greater than 50,000 gal of drainable interstitial liquid, and in two DSTs only. The LOWs installed in two DSTs (102-SY and 103-AW Tanks only) are used for special surveillance purposes only.

A thermocouple is a thermoelectric device used to measure temperature. More than one thermocouple on a device (probe) is called a thermocouple tree. In DSTs there may be one or more thermocouple trees in 


\section{WHC-EP-0182-56}

risers in the primary tank. In addition, in DSTs only, there are thermocouple elements installed in the insulating concrete, the lower primary tank knuckle, the secondary tank concrete foundation, and in the outer structural concrete. These monitor temperature gradients within the concrete walls, bottom of the tank, and the domes. In SSTs, there may be one or more thermocouple trees installed directly in a tank, although some SSTs do not have any trees installed. A single thermocouple may be installed in a riser, or lowered down an existing riser or LOW. There are also four thermocouple laterals beneath Tank 105-A in which temperature readings are taken in 34 thermocouples.

In-tank

Photography

In-tank photographs may be taken to aid in resolving in-tank measurement anomalies and determine tank integrity. Photographs help determine sludge and liquid levels by visual examination.

\section{INVENTORY AND STATUS BY TANK - COLUMN CALCULATIONS (SINGLE-SHELL TANKS)}

\section{COLUMN HEADING}

Total Waste Solids Volume plus Supernatant liquid.

Supernatant Liquid

Drainable Interstitial

Total Jet

Pumped

Drainable

Liquid

Remaining

Pumpable

Liquid

Remaining

Sludge
Drainable Liquid Remaining minus Drainable Interstitial. Supernate is usually derived by subtracting the solids level measurement from the liquid level measurement.

Drainable Liquid Remaining minus Supernate.

Drainable Interstitial Liquid is calculated based on the saltcake and sludge volumes, using average porosity values or actual data for each tank, when available.

Cumulative total pumped 1979 to date.

Supernate plus Drainable Interstitial.

Drainable Liquid Remaining less undrainable heel volume.

Solids formed during sodium hydroxide additions to waste. Sludge usually was in the form of suspended solids when the waste was originally received in the tank from the waste generator. In-tank photographs may be used to estimate the volume. 
Saltcake Results from crystalization and precipitation after concentration of liquid waste, usually in an evaporator. If saltcake is layered over sludge, it is only possible to measure total solids volume. In-tank photographs may be used to estimate the saltcake volume.

Solids Volume Indicates the latest update of any change in the solids Update volume.

Solids Update Indicates the source or basis of the latest solids Source - See volume update.

Footnote

Last Photo Date of latest in-tank photographs taken.

Date

Change Since Indicates any change made since the previous month. Last Monthly Explanation for the change follows the Inventory and Report Status by Tank section. 
WHC-EP-0182-56

\section{APPENDIX B}

TANK FARM CONFIGURATION, STATUS, AND

FACILITY CHARTS 
WHC-EP-0182-56

This page intentionally left blank. 

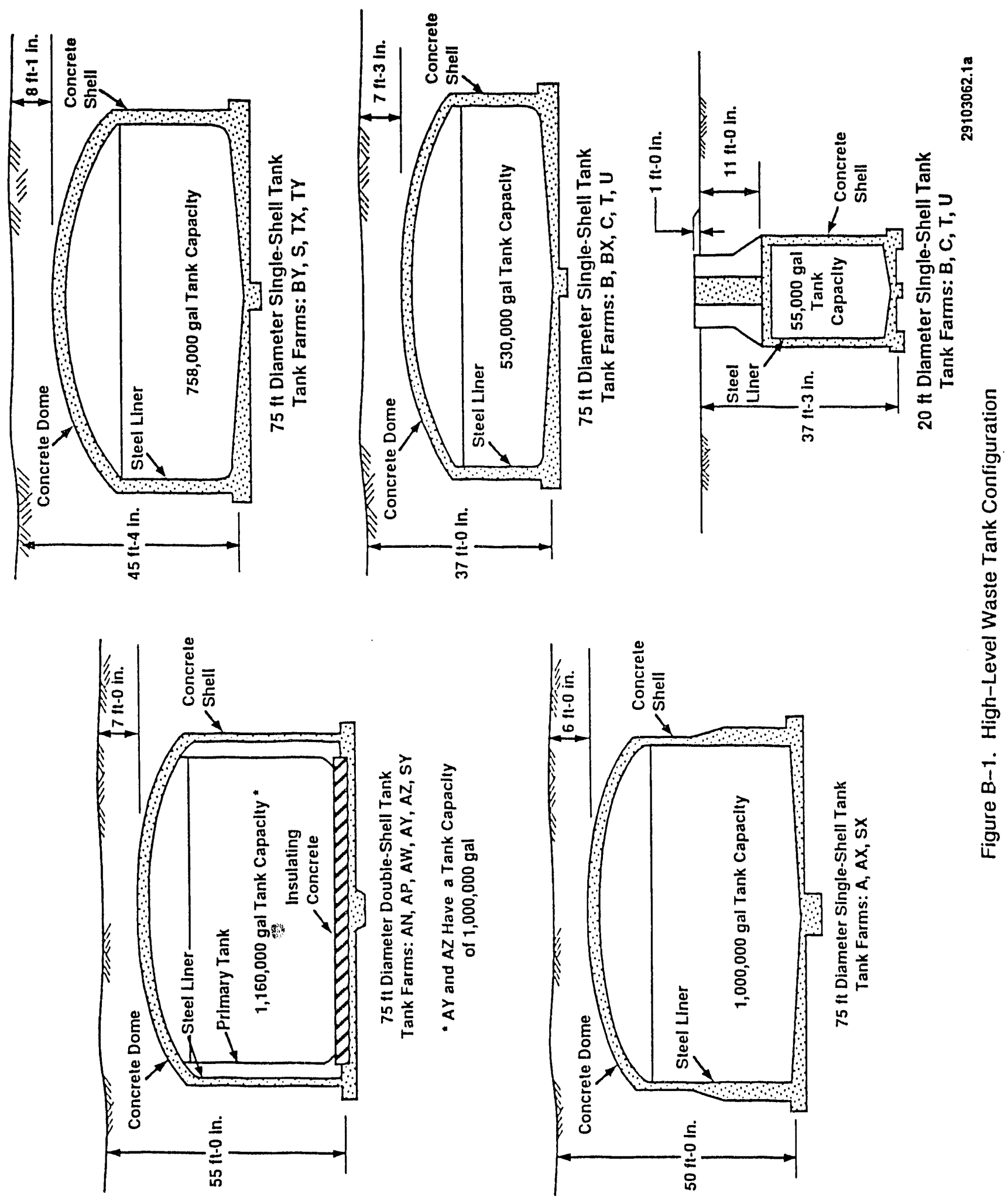

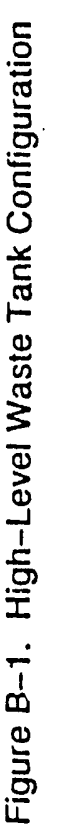




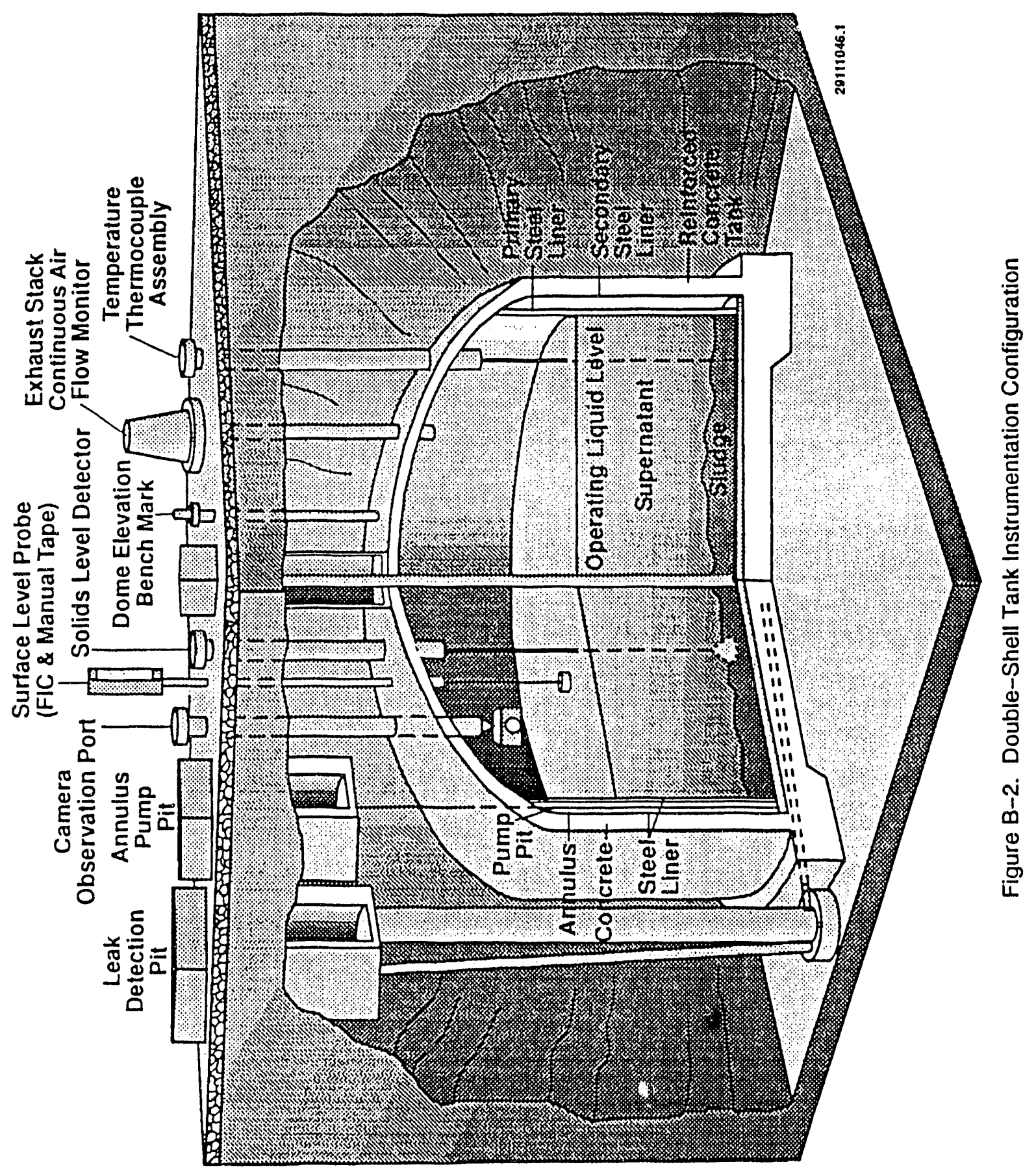


WHC-EP-0182

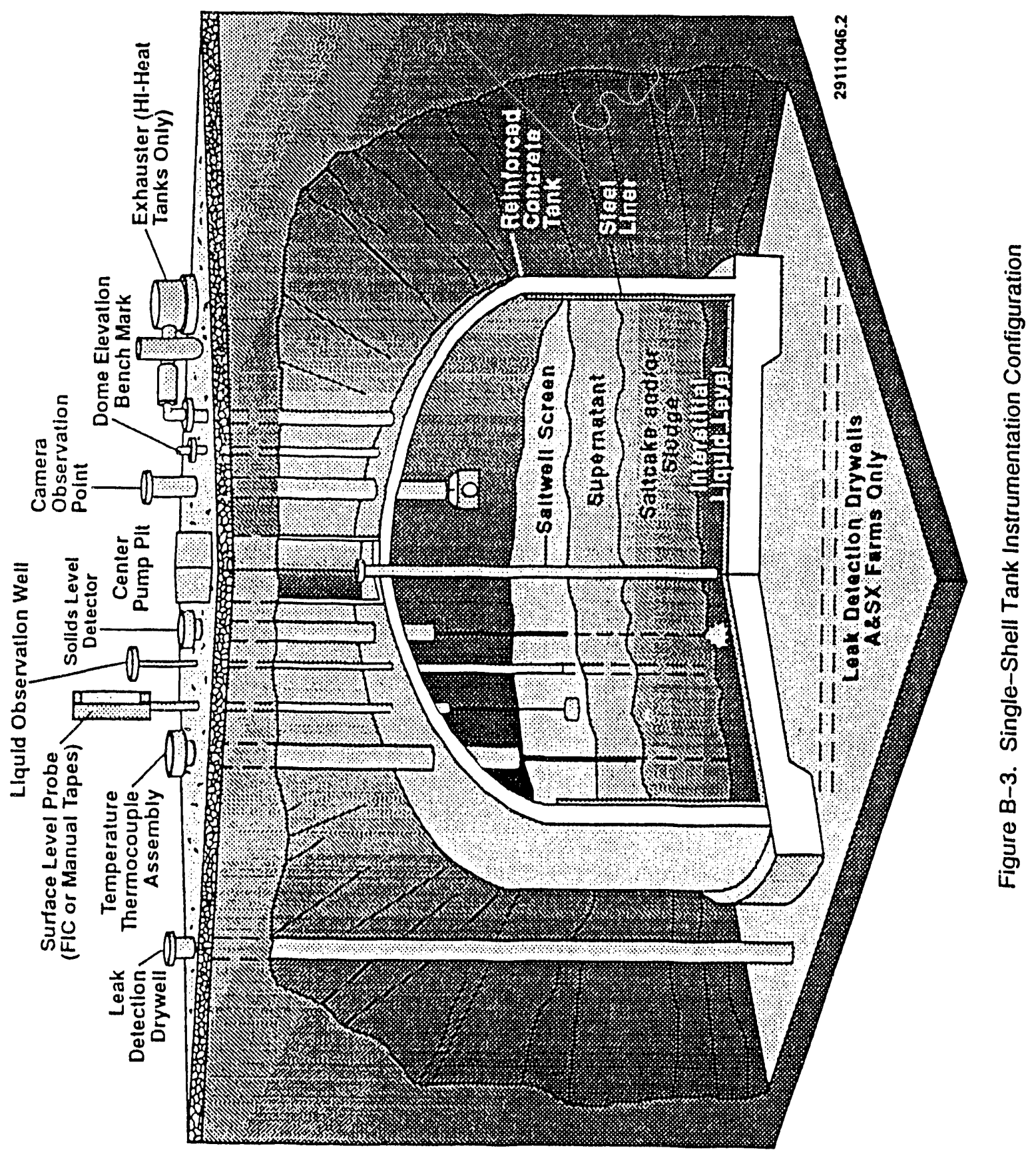

B-5 
WHC-EP-0182

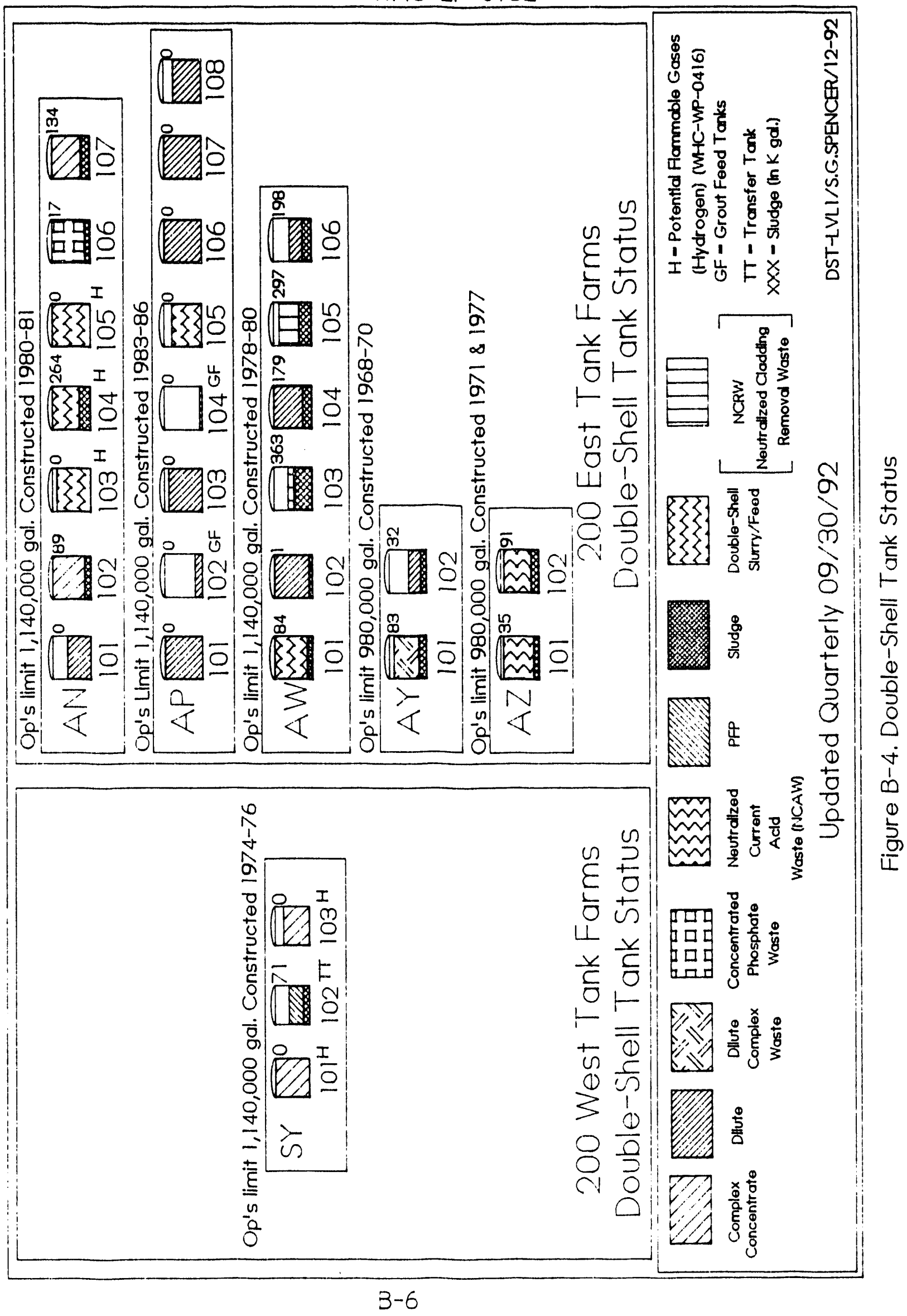


WHC-EP-0182

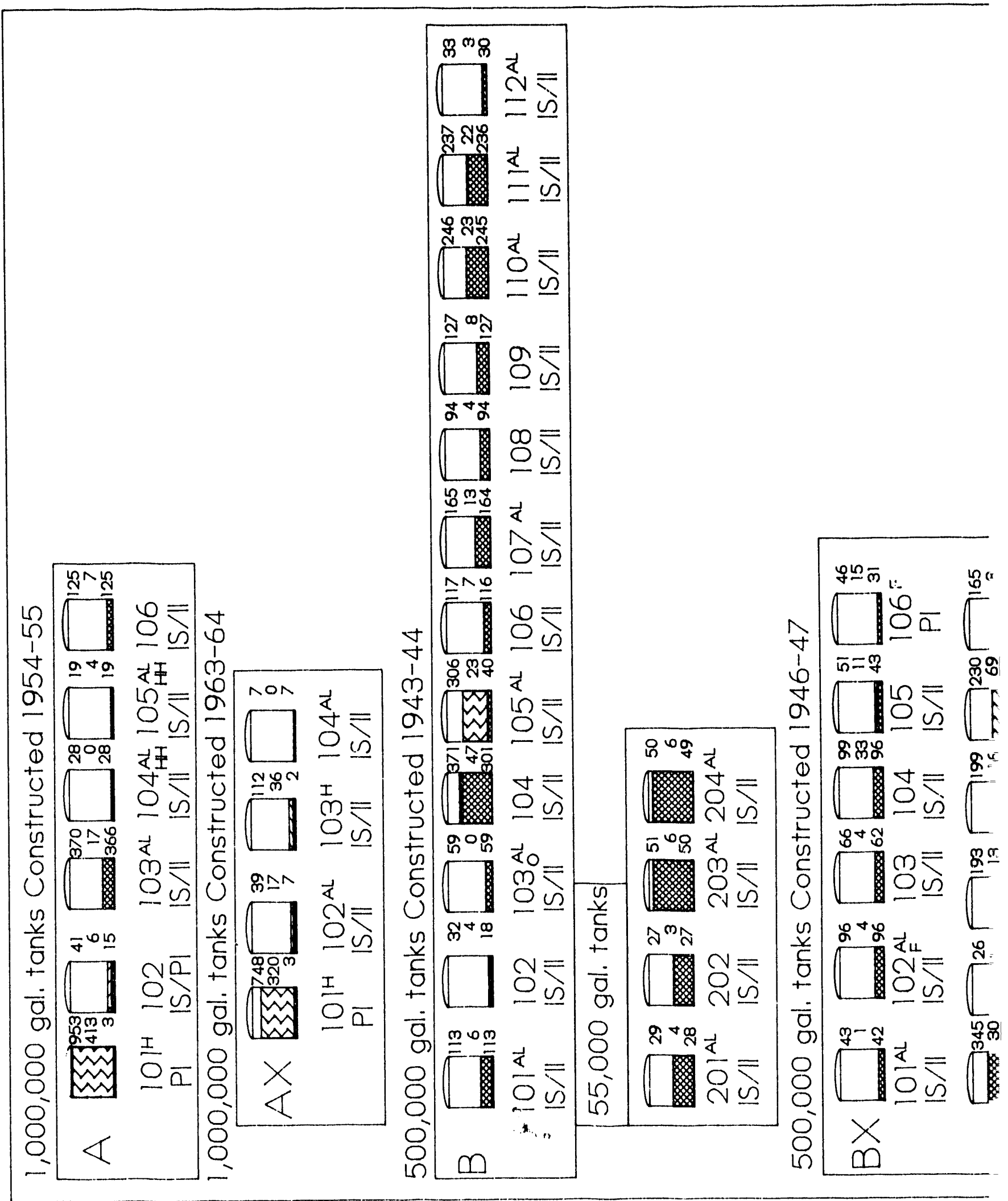



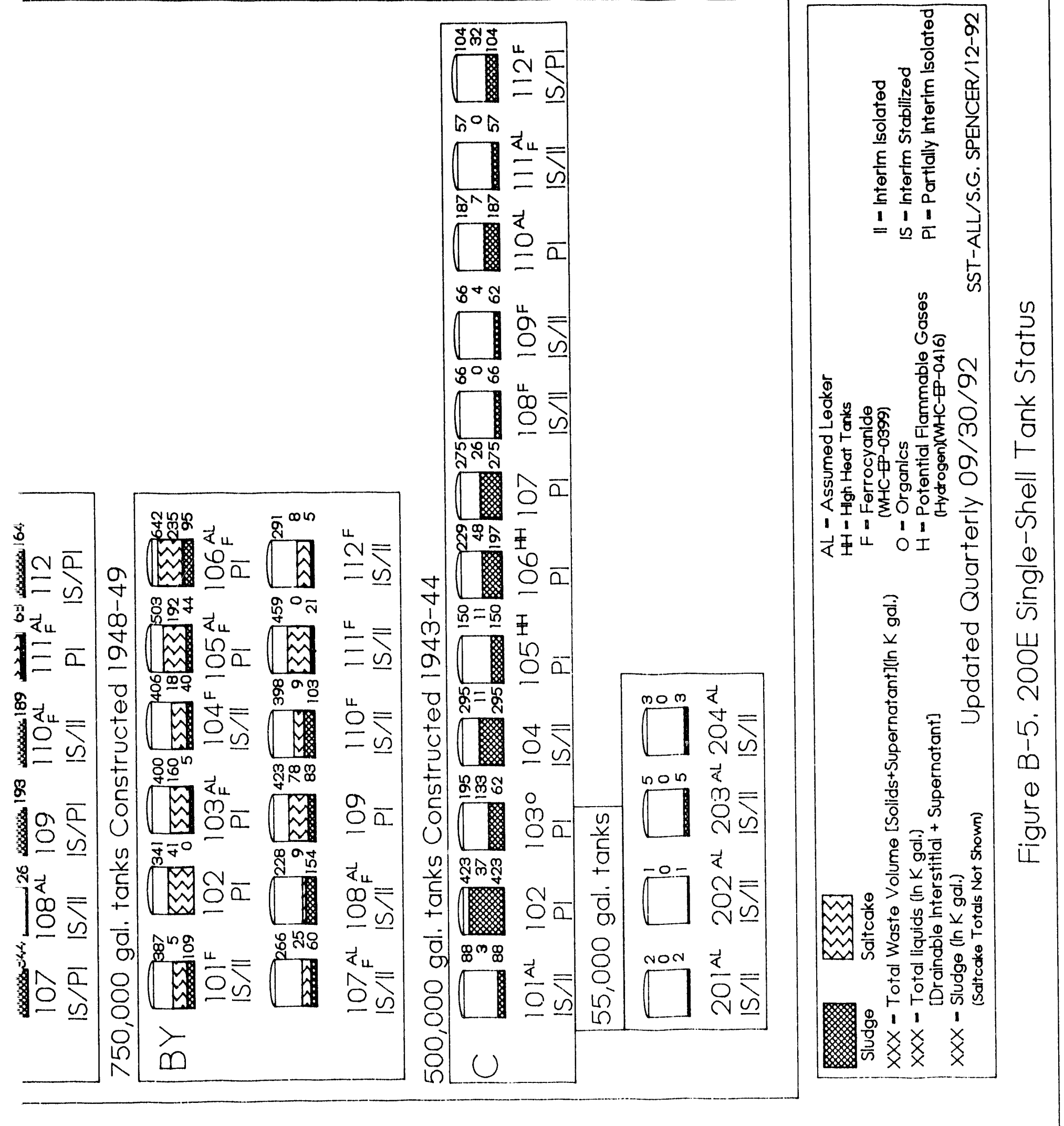

$B-7 / 8$ 
WHC-EP-0182

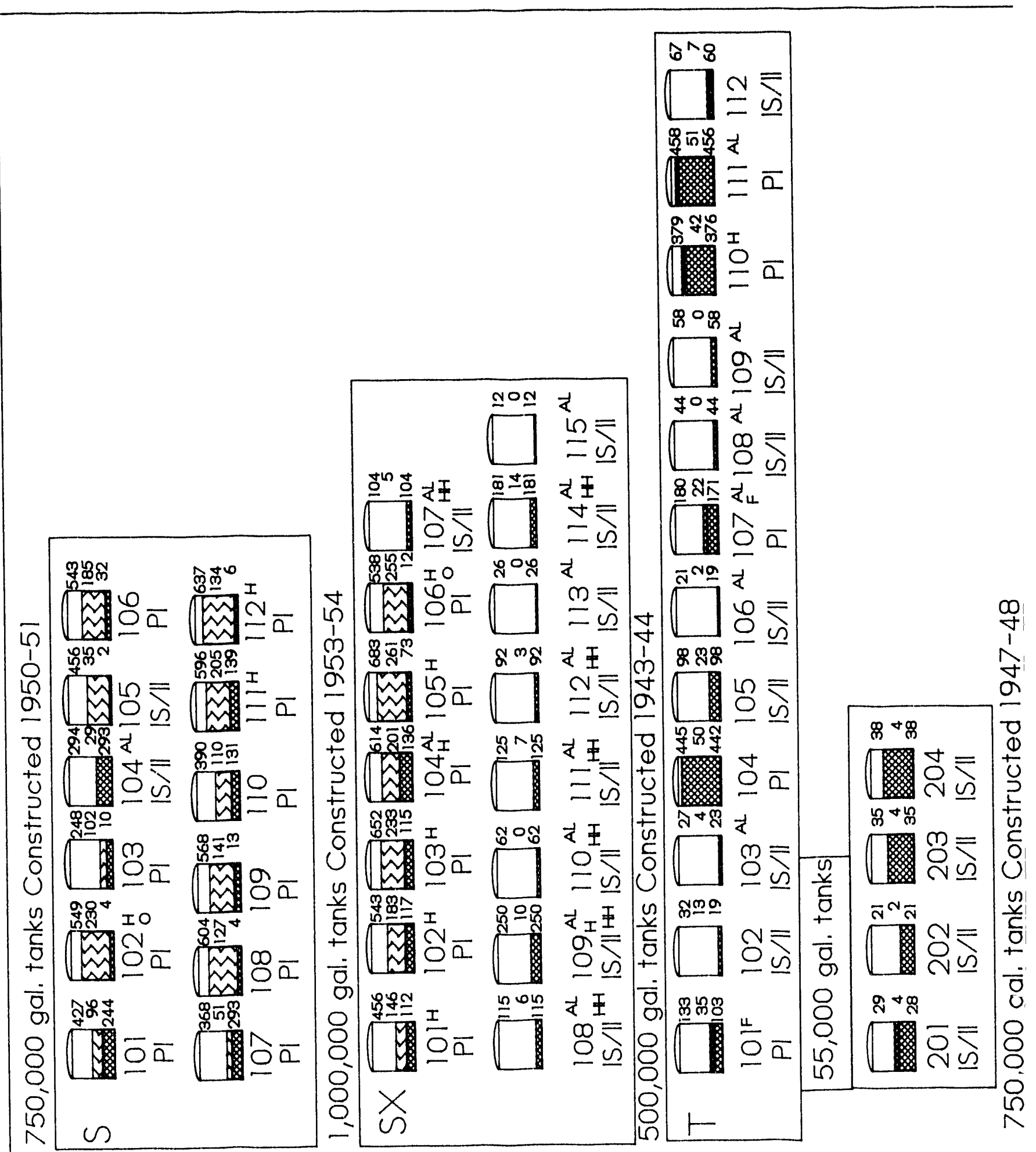



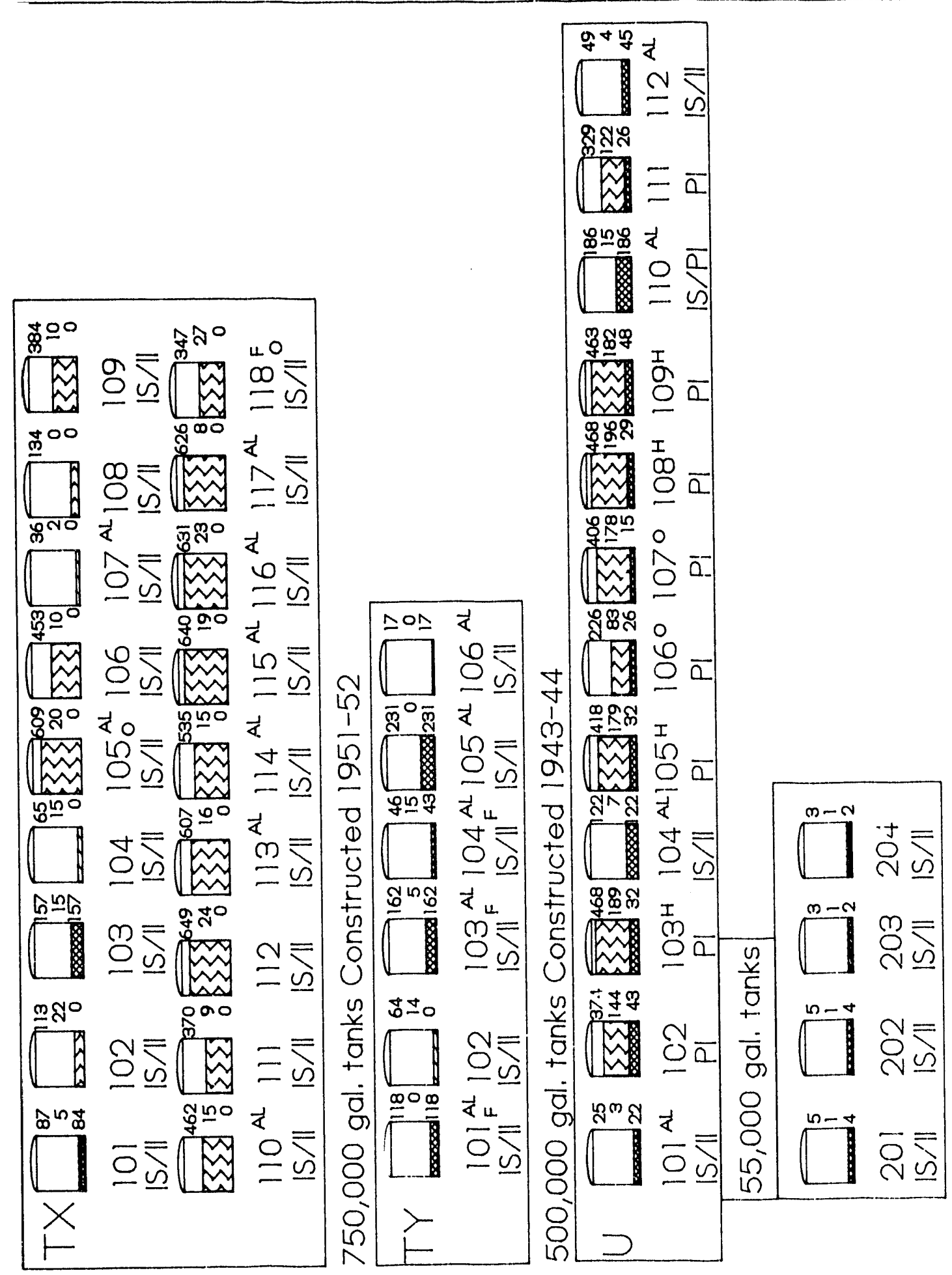


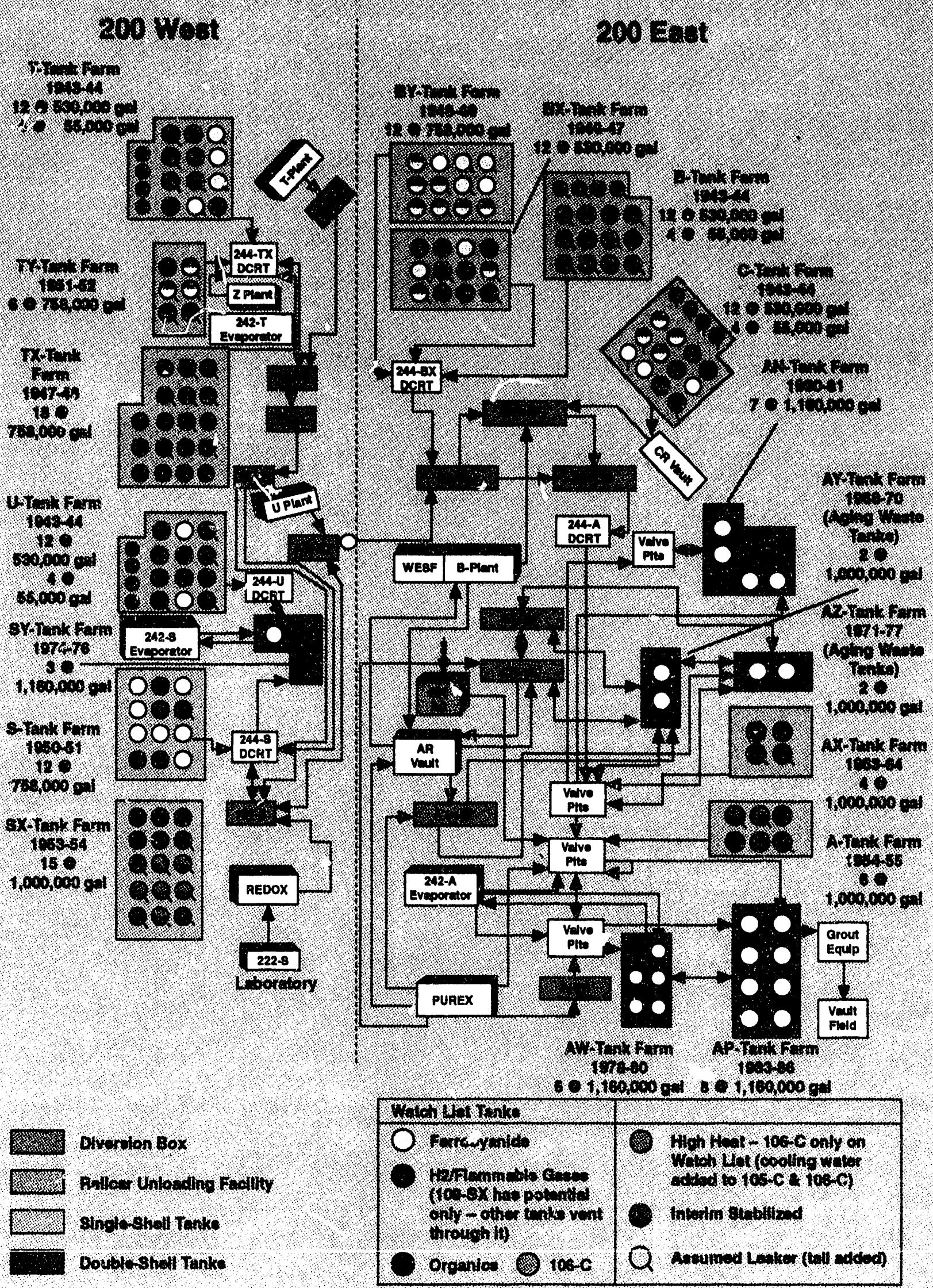

$29212049,1 \mathrm{C}$

Figure B-7. Tank Farm Facilities - Quick Reference 


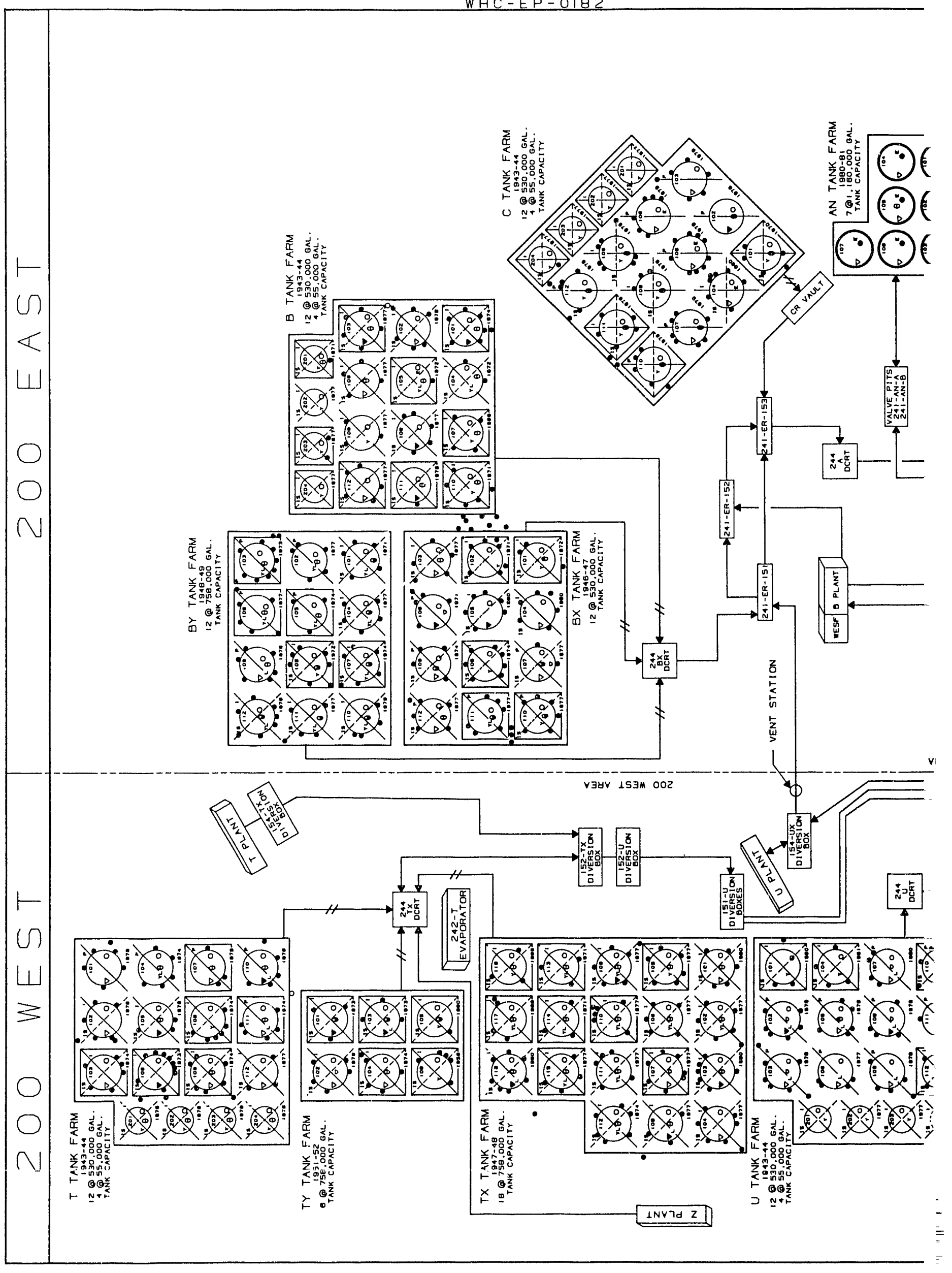




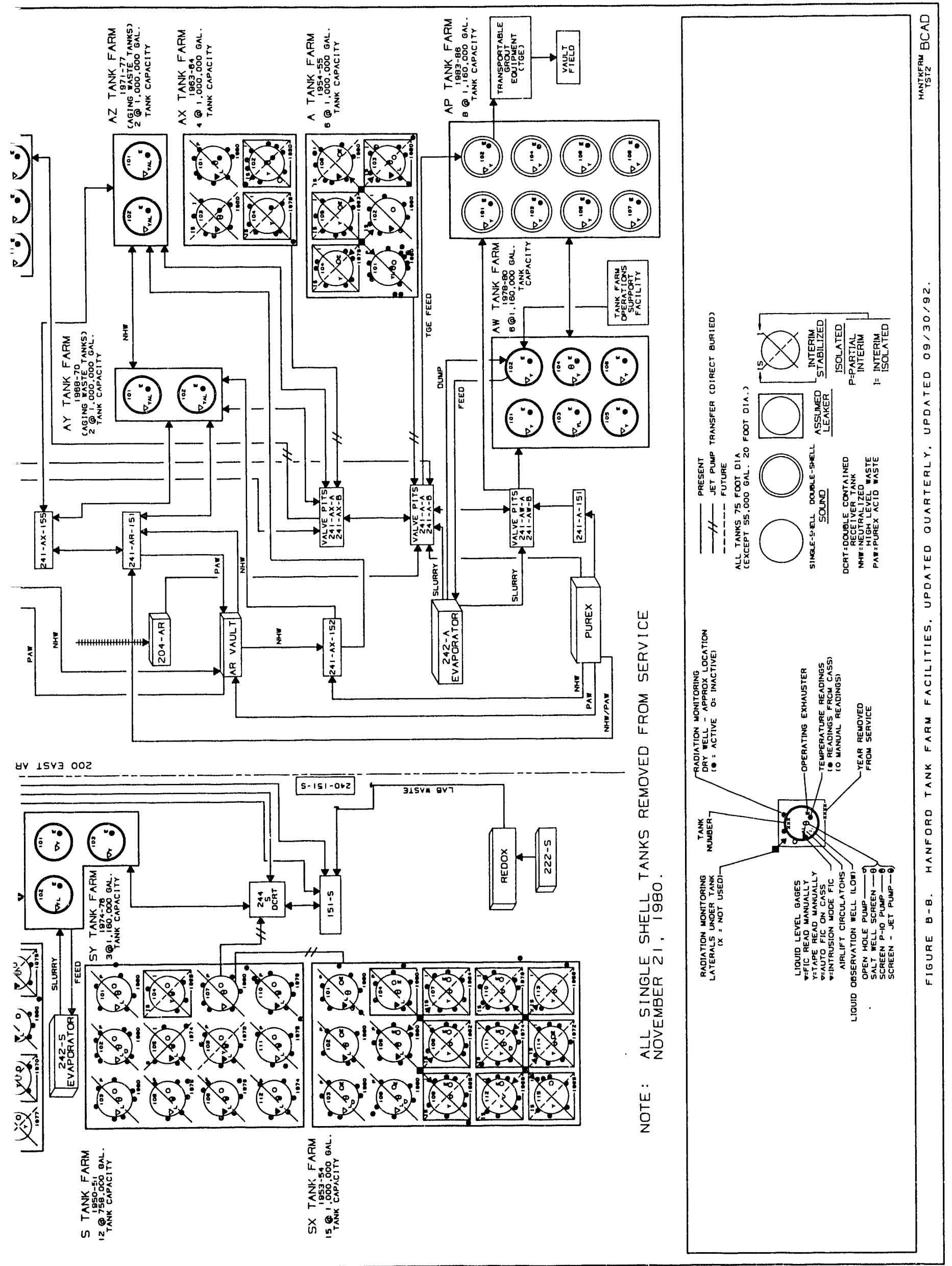


WHC-EP-0182-56

\section{APPENDIX C}

\section{MONTHLY SUMMARY}

TANK USE SUMMARY

INVENTORY SUMMARY BY TANK FARM INVENTORY AND STATUS BY TANK 
WHC-EP-0182-56

This page intentionally left blank. 


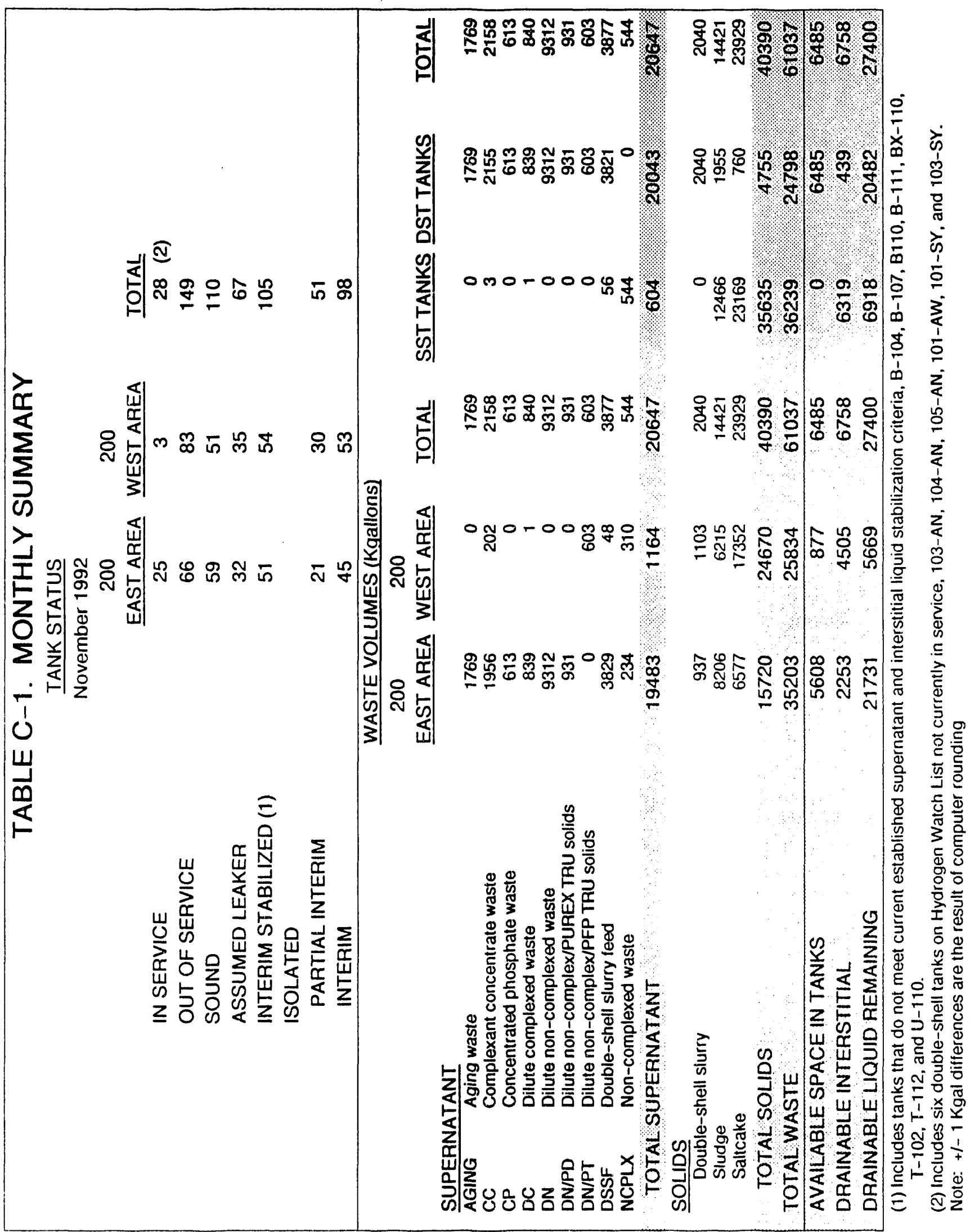




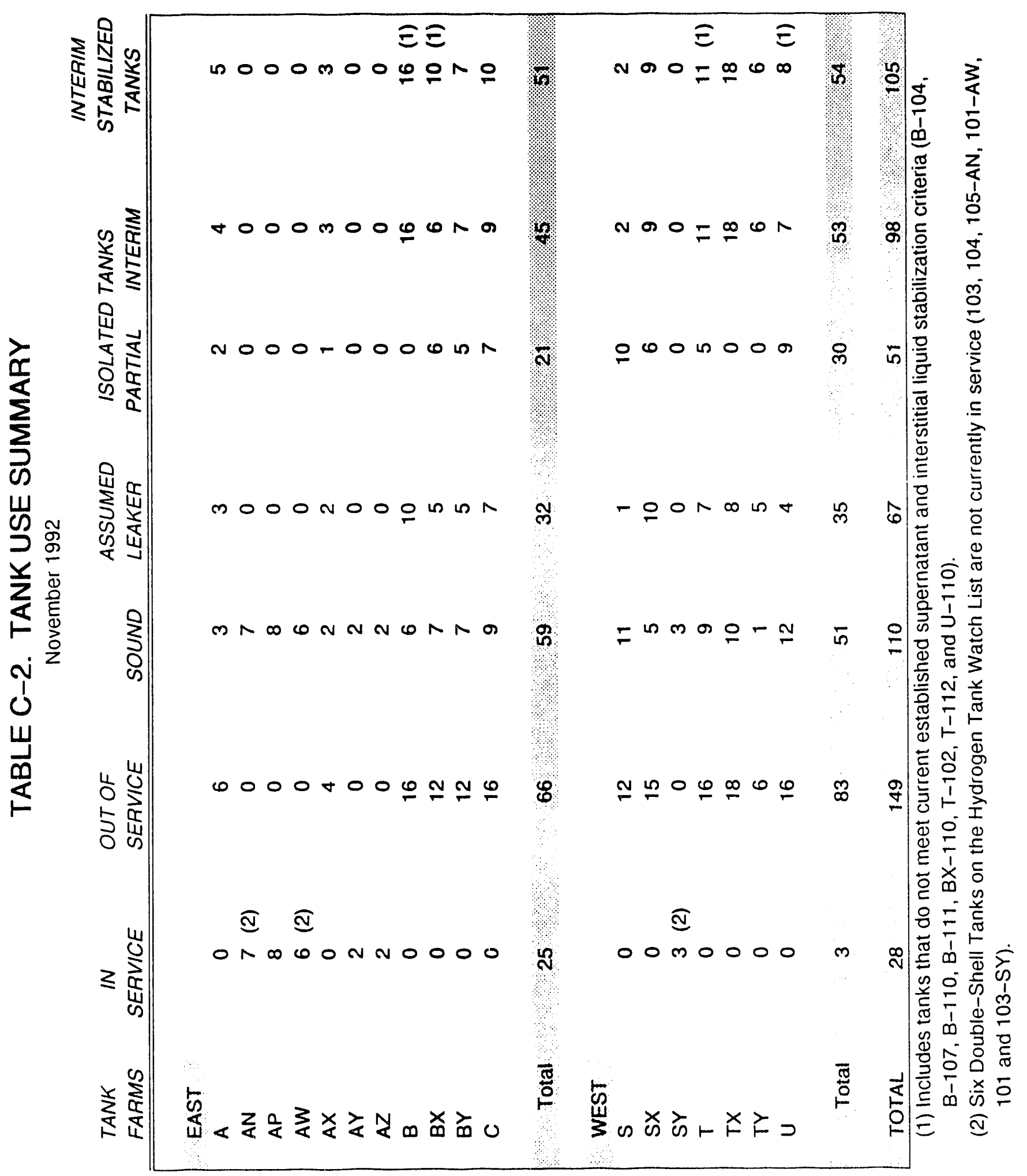




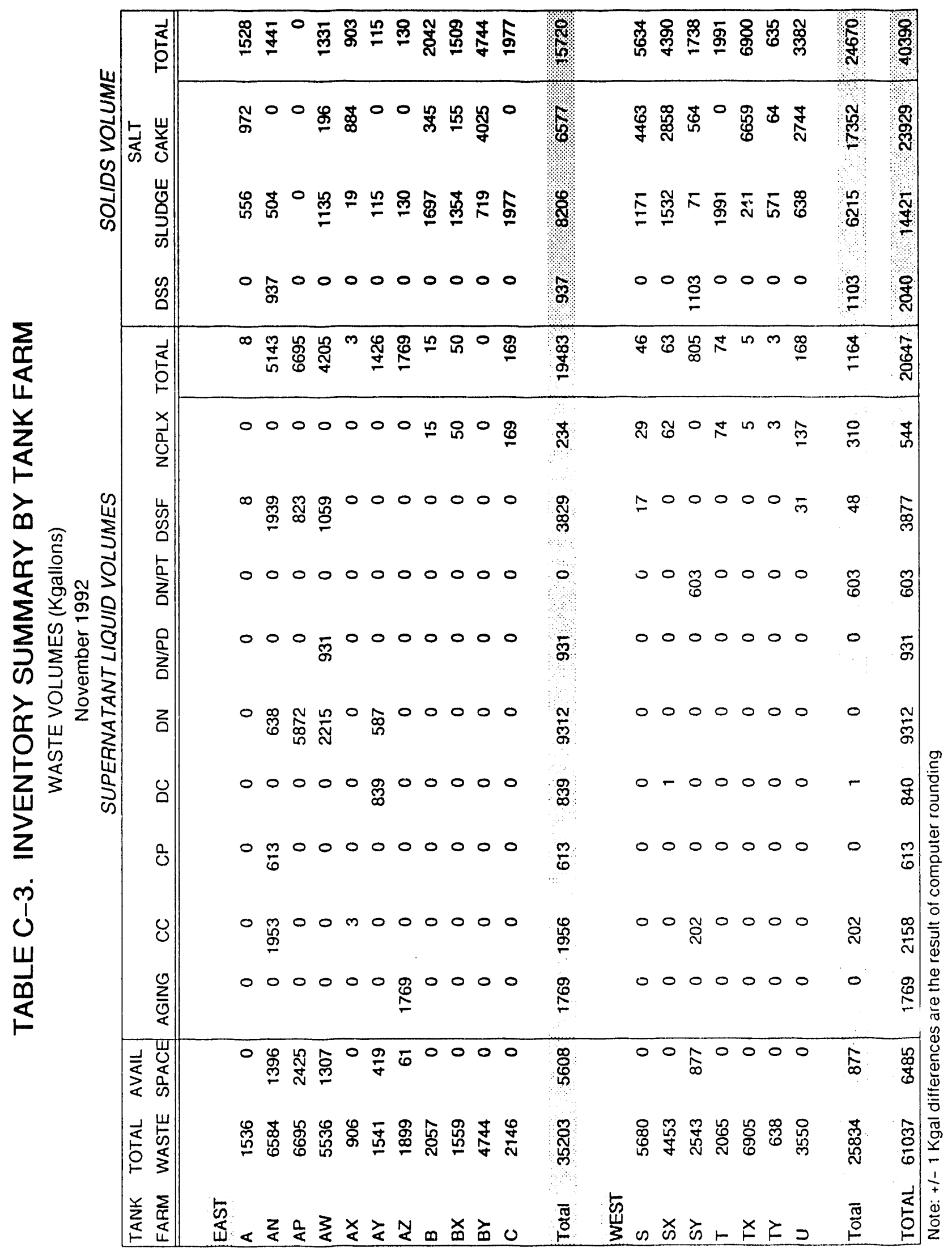




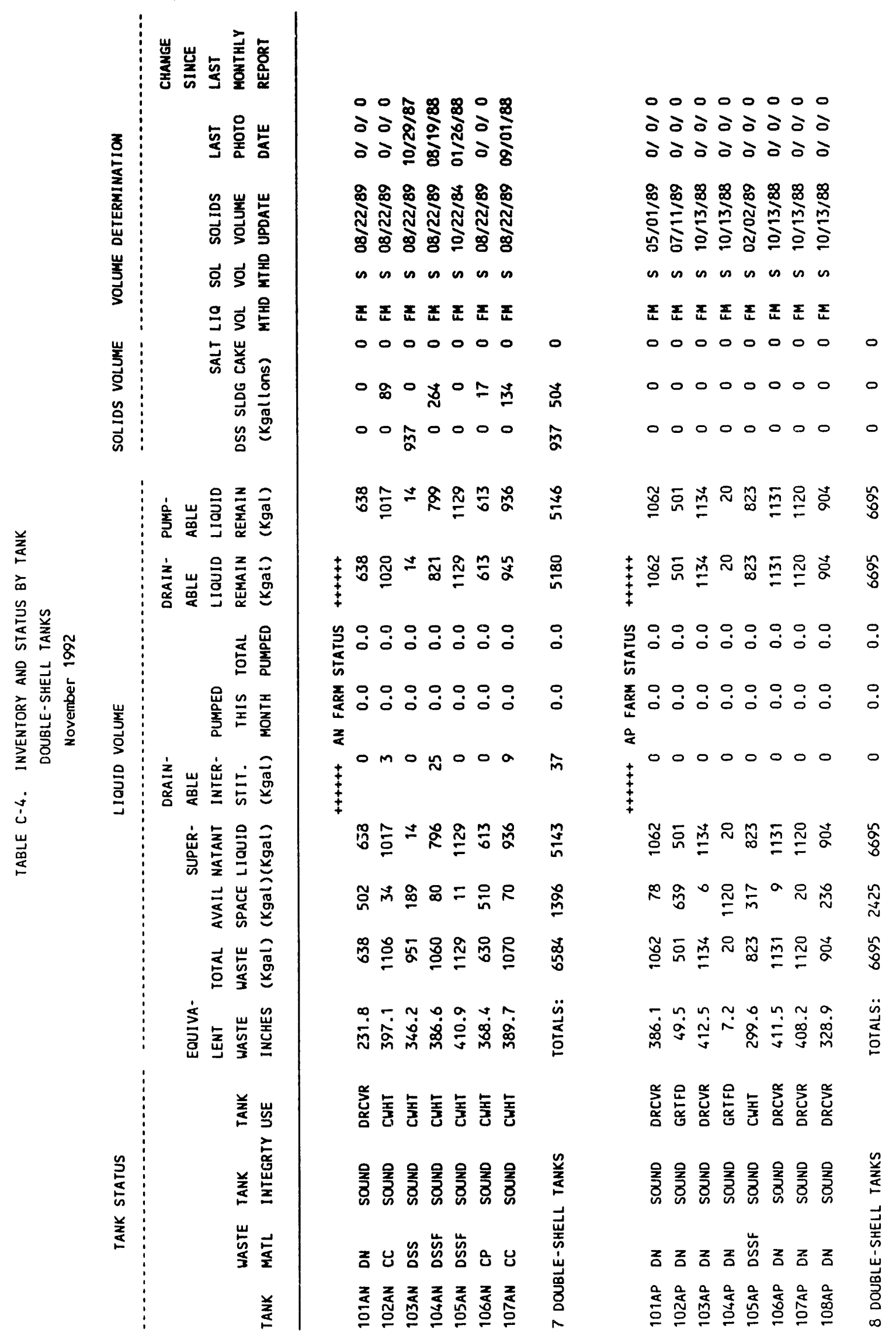


WHC-EP-0182-56

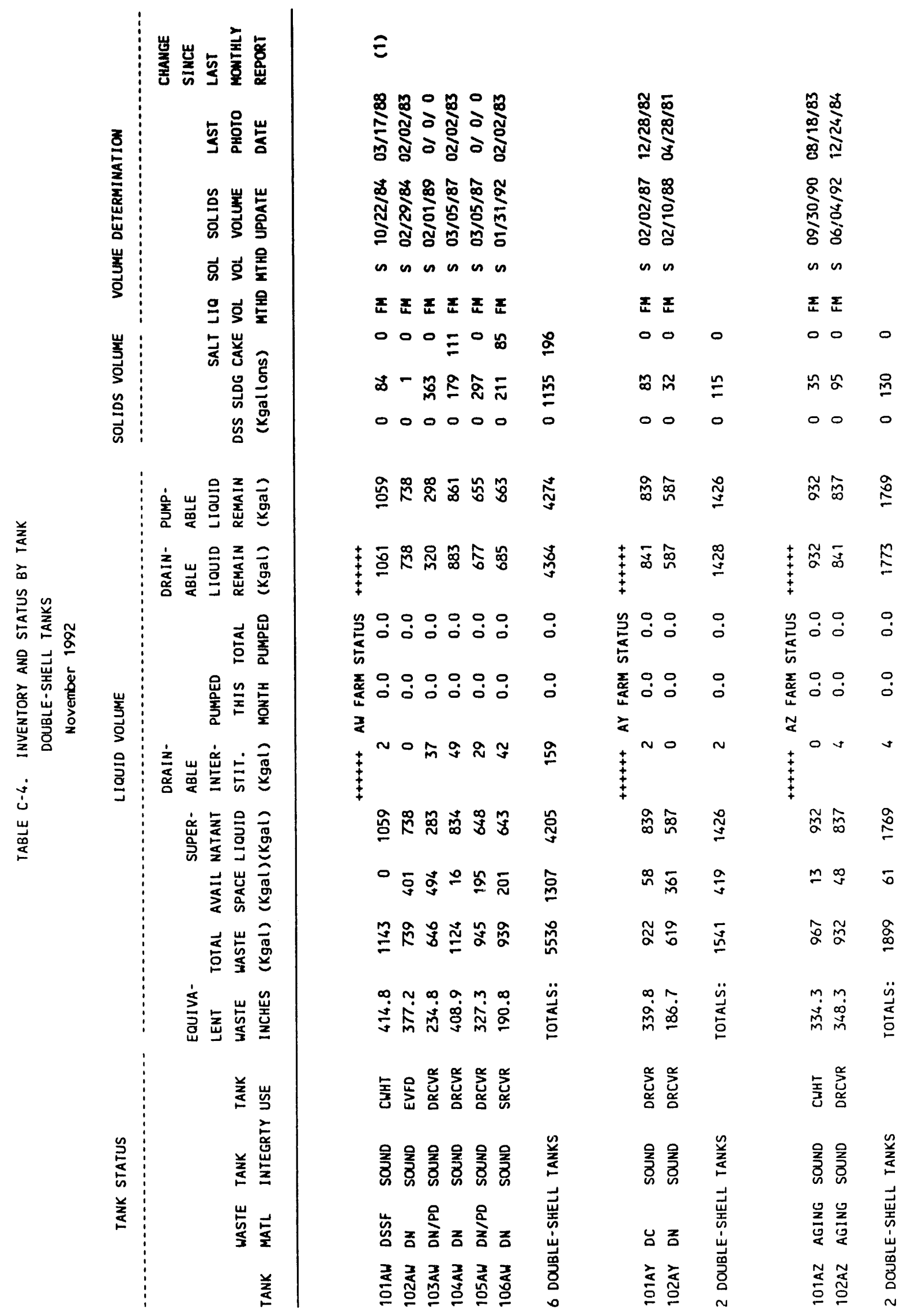




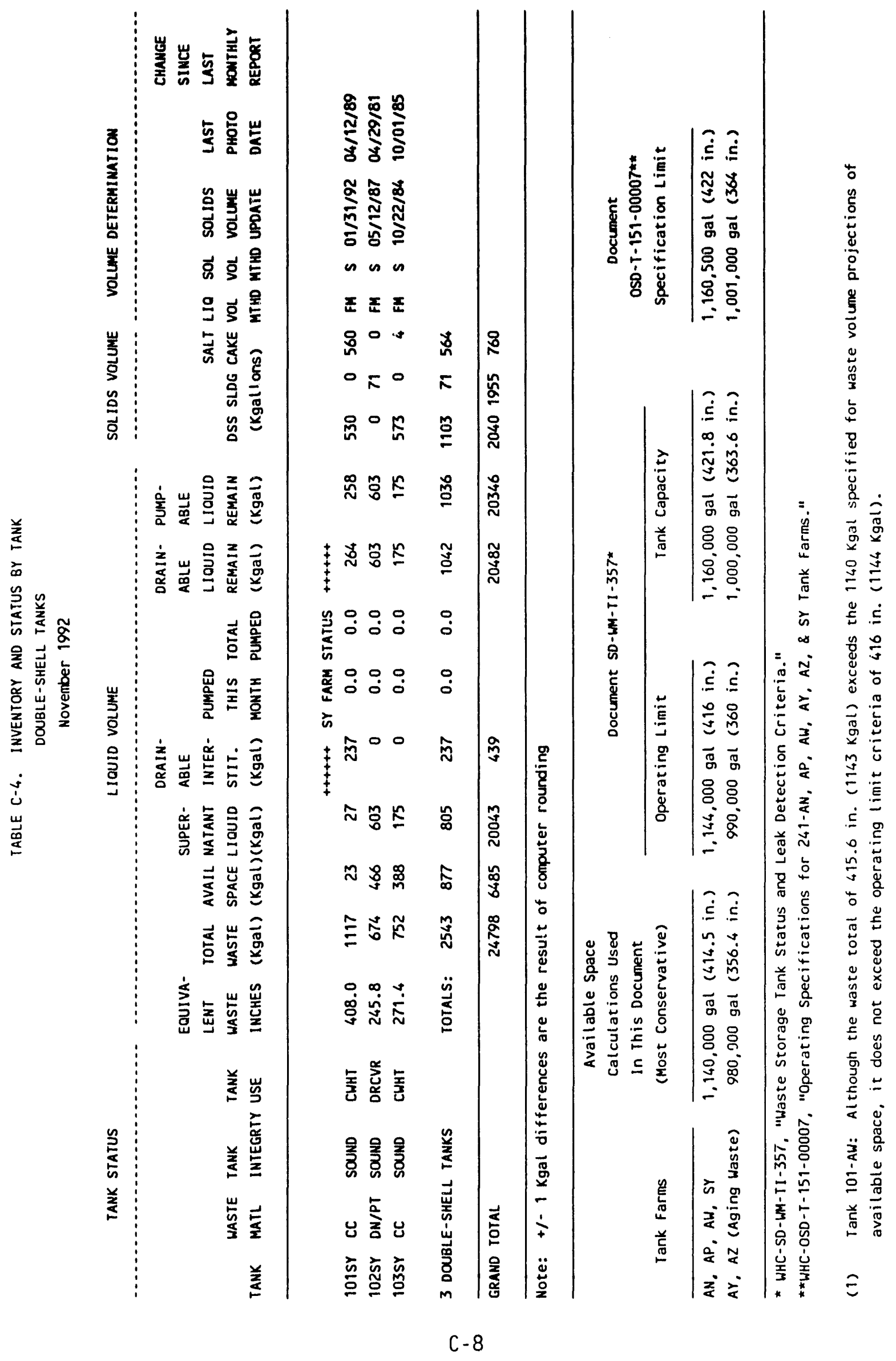




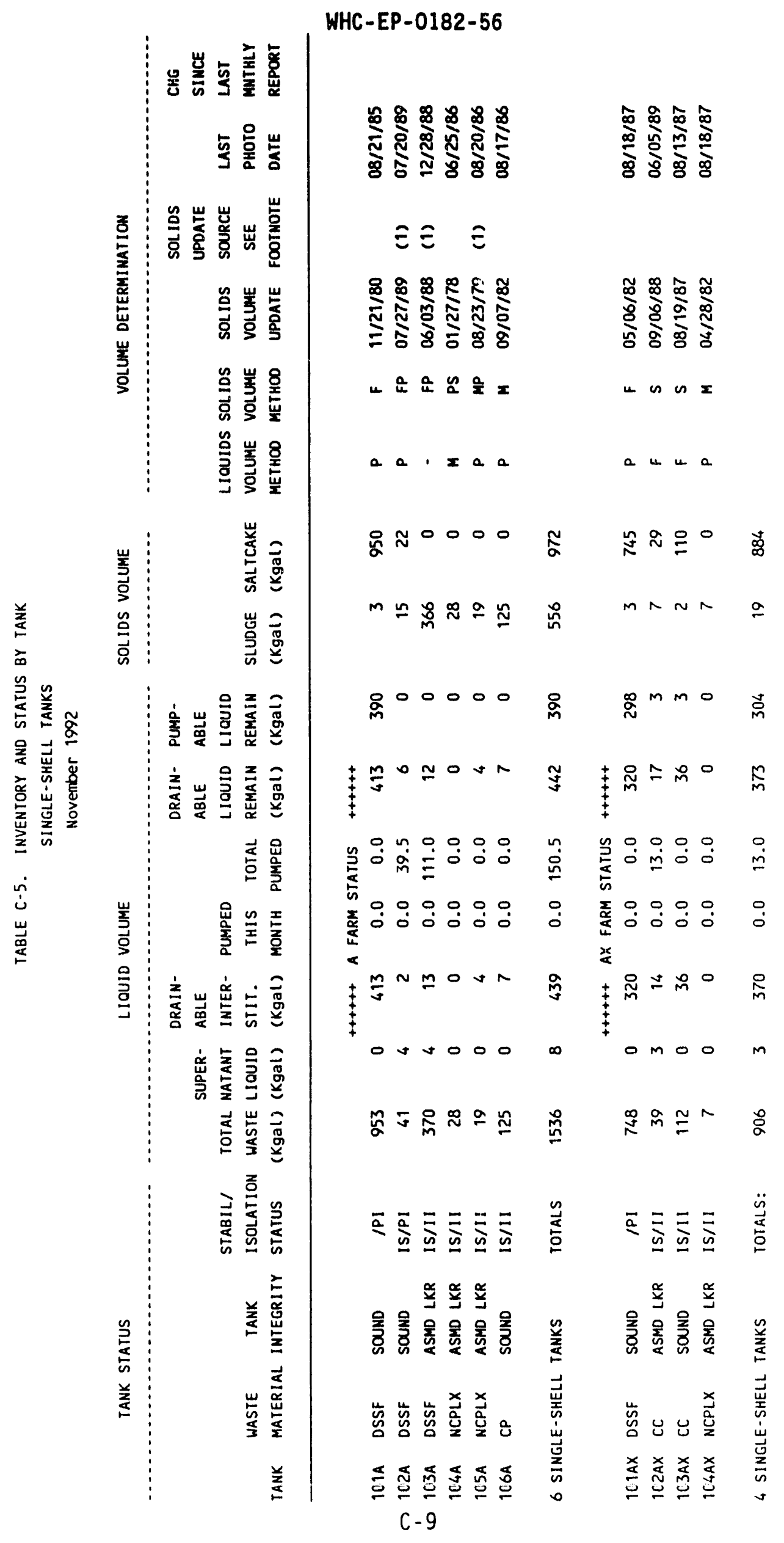


号訔营重喜

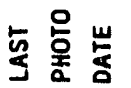

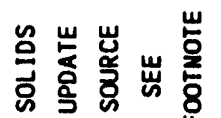

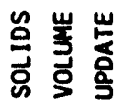

苍崖要

岂崖部

崖

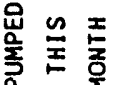

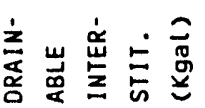

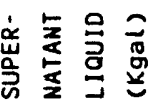

喜容总

要总亮

美惫

差

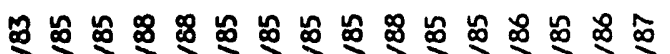

일

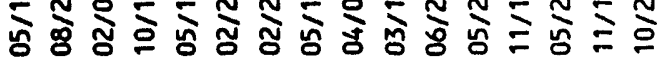

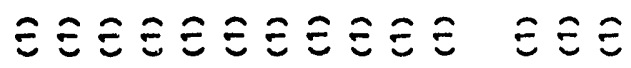

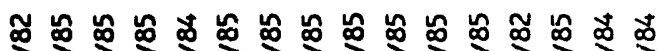

क्ष

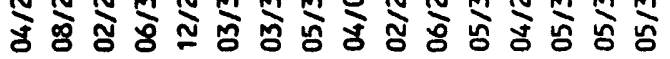

UUU

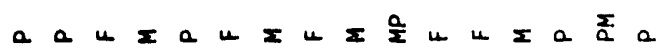

0909800000000000 鬲

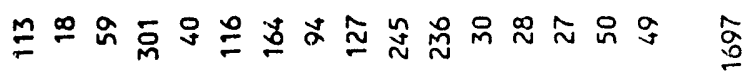

OOOgOONOONDOOODO D

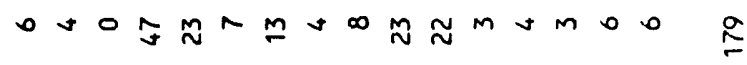

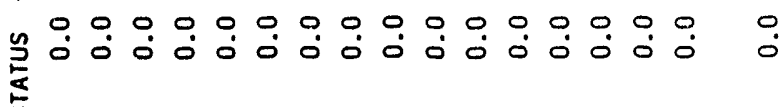

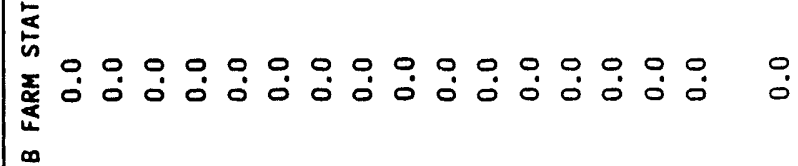

.

ovo-o-no-m-0-.

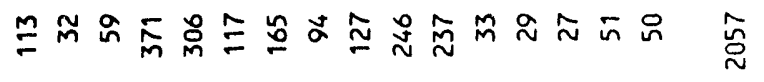

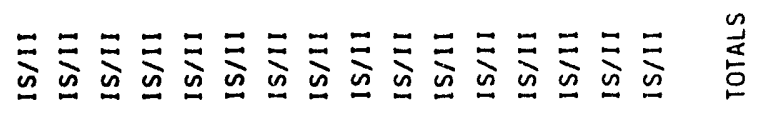

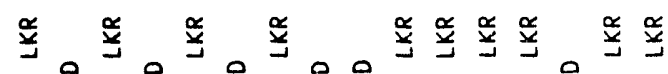

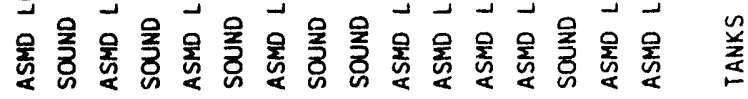

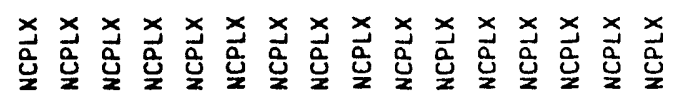

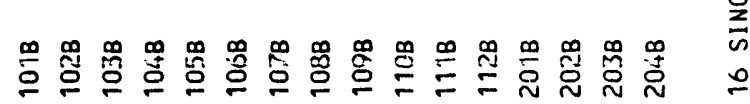
C- 10 


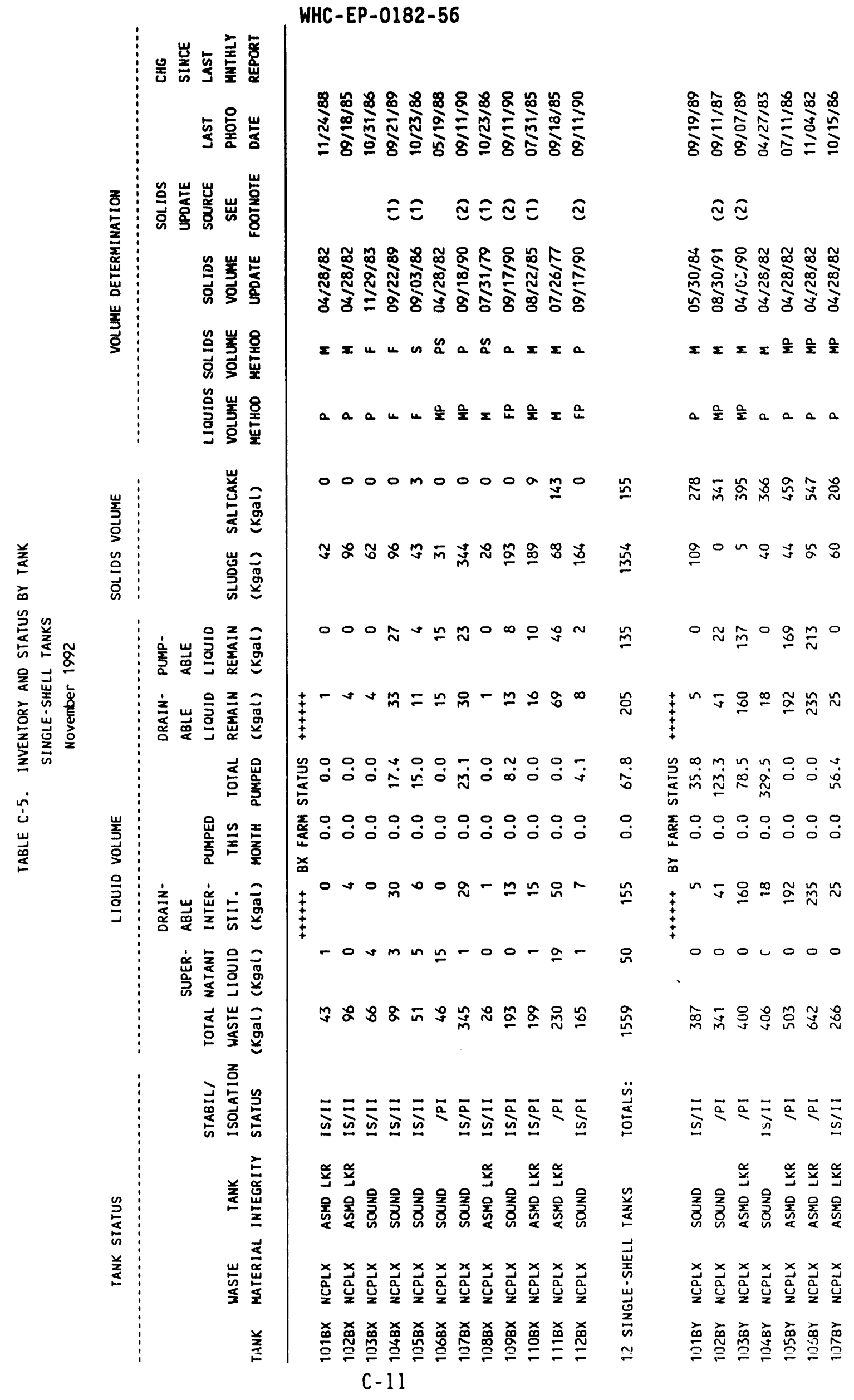




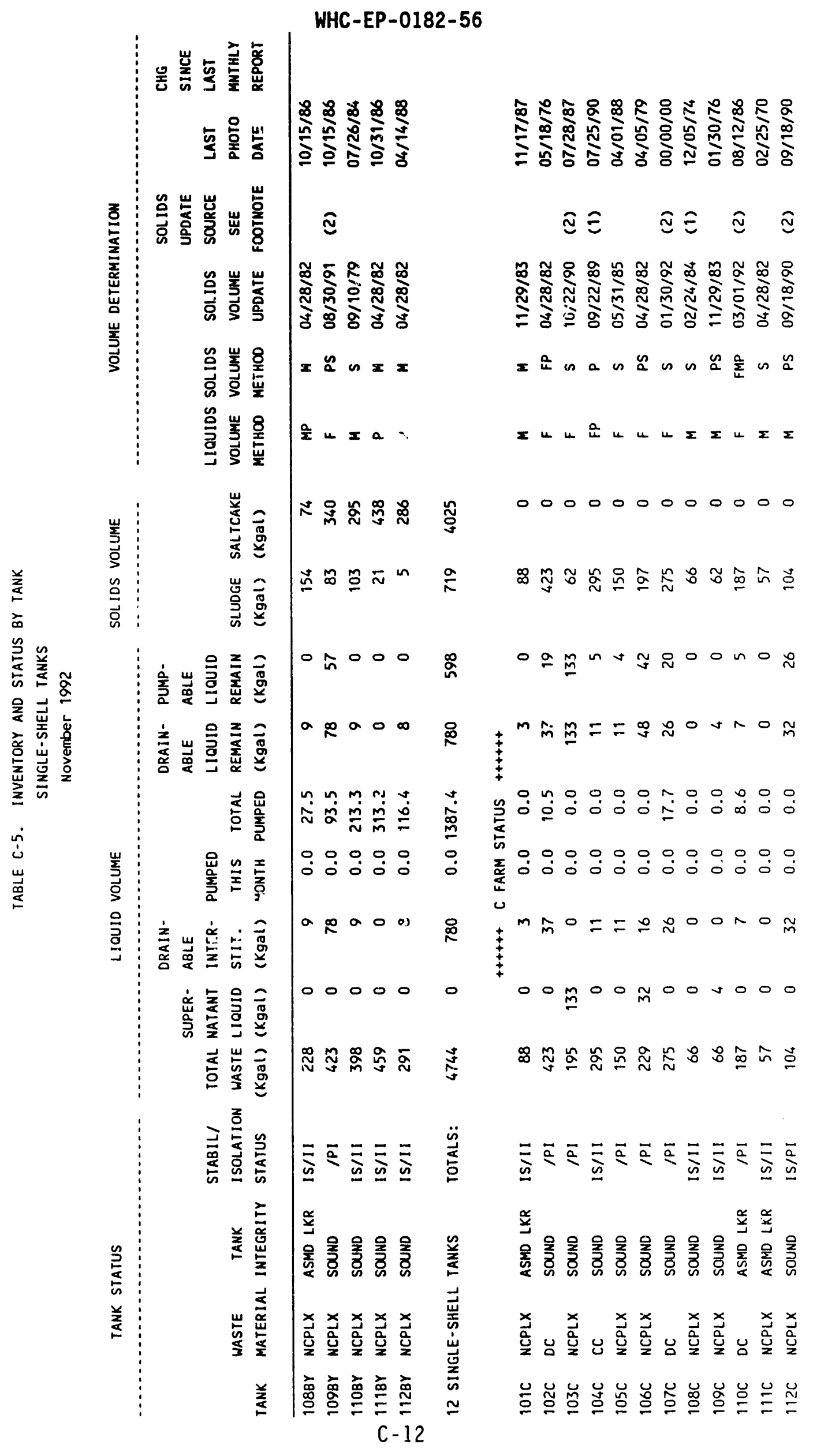




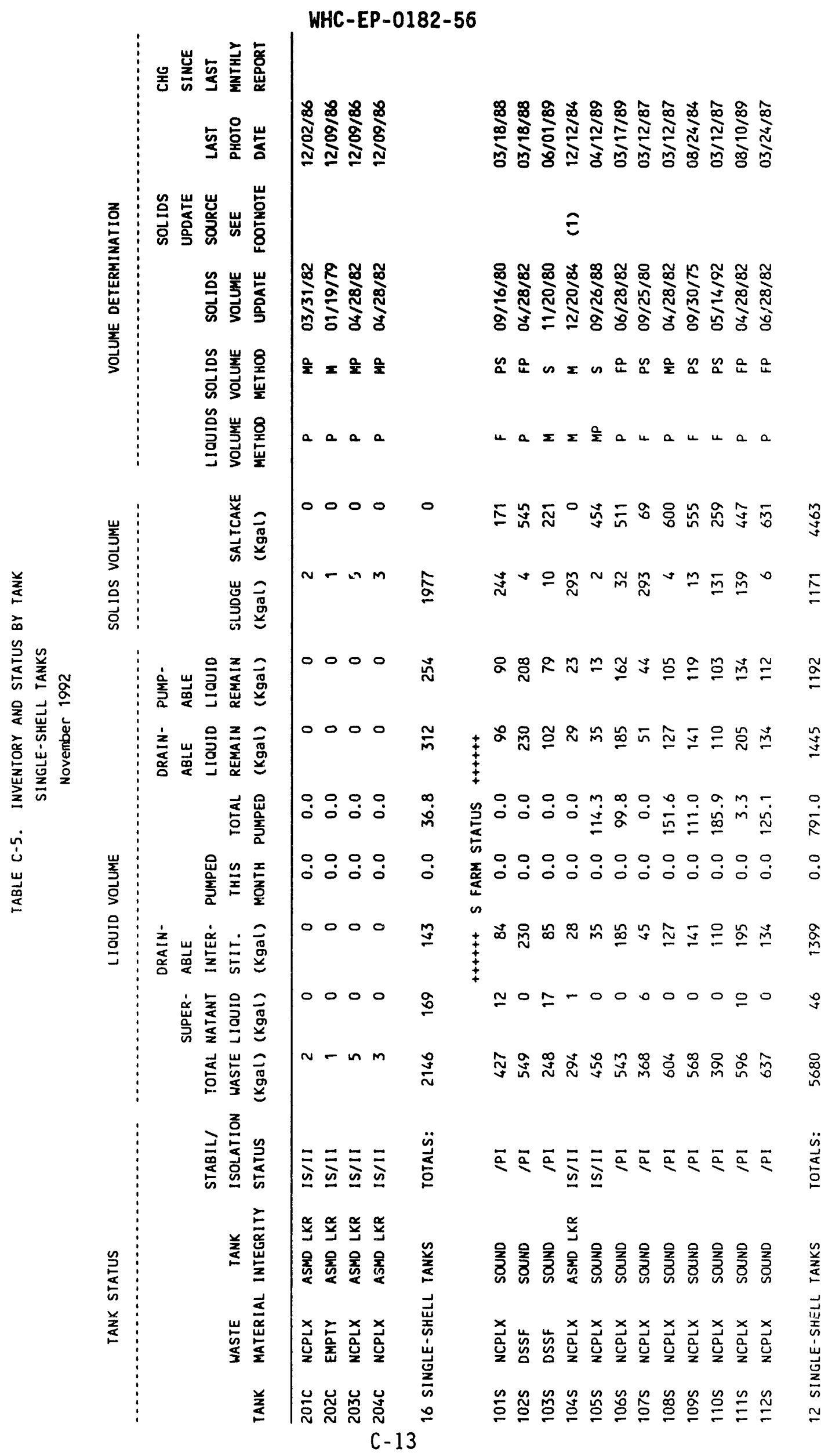




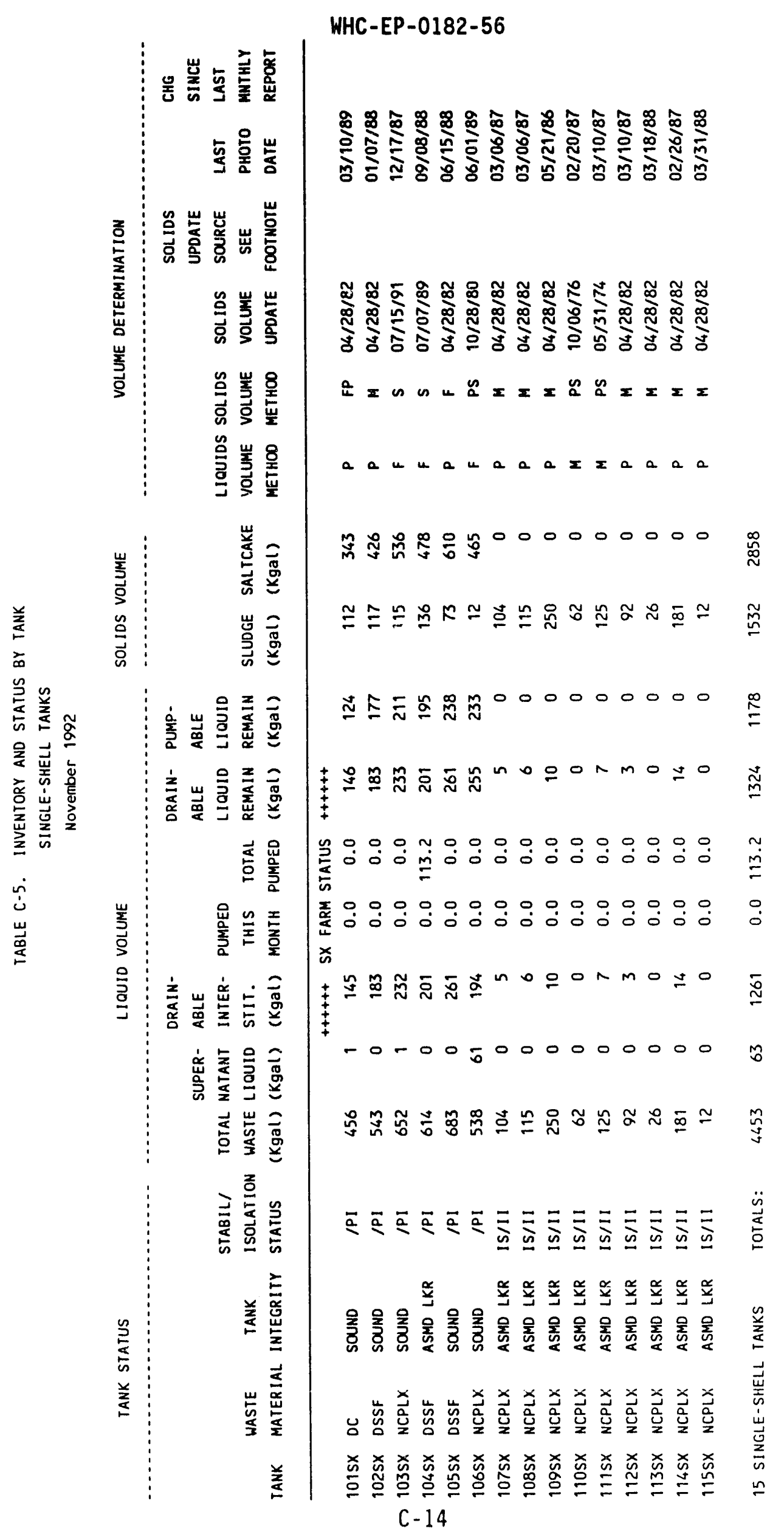


高䇾宸

竞:

西

悹美。

웅 吉

产志总

焉

i

状

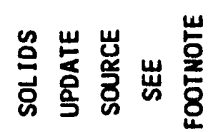

岂崖

岂崖

总 壱

苍崖 8

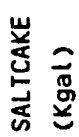

岂苛

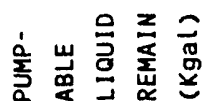

表容

崖

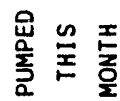

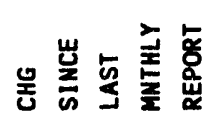

WHC-EP-0182-56

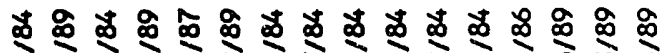

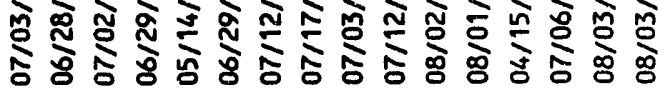

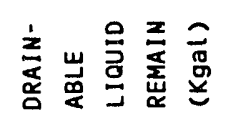

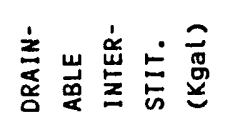

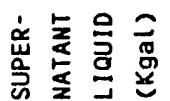

崖苫离

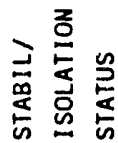

$\Xi$

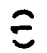

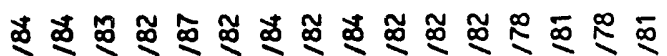

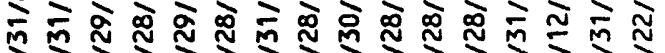

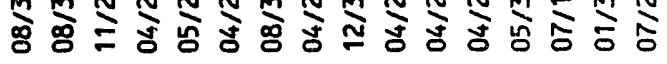

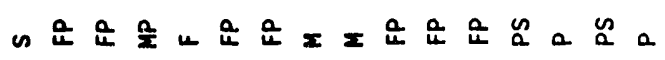

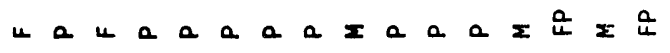

00000000000000000

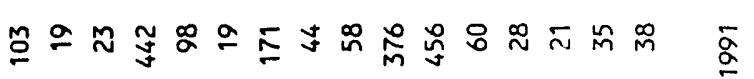

을

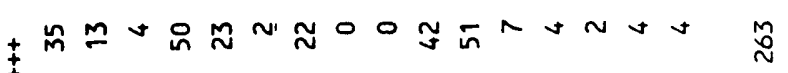

I 丕

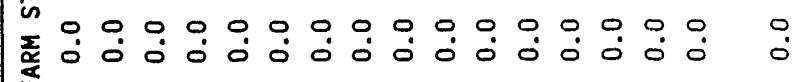
(2)

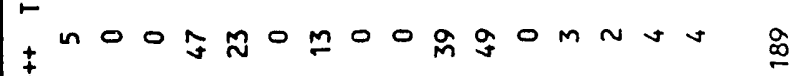
$\dot{+}$

m m M M N O OOMNN-000 N

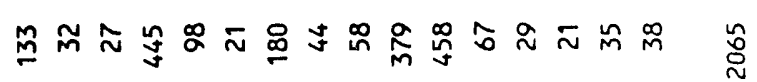

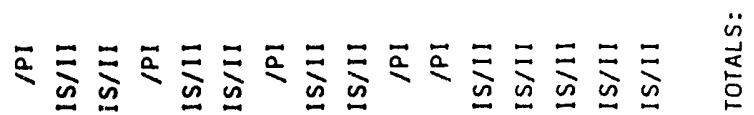

美怘

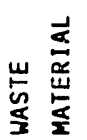

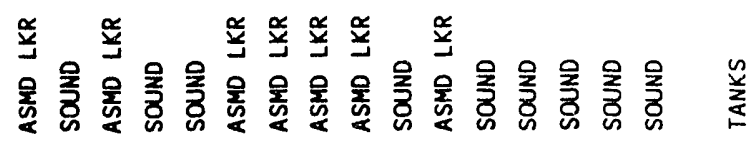

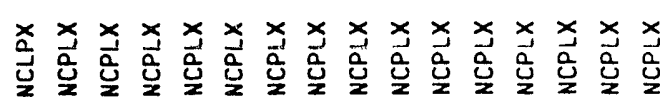

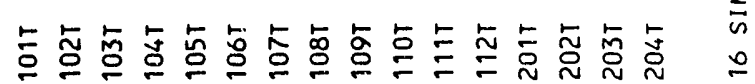

$$
\text { C- } 15
$$




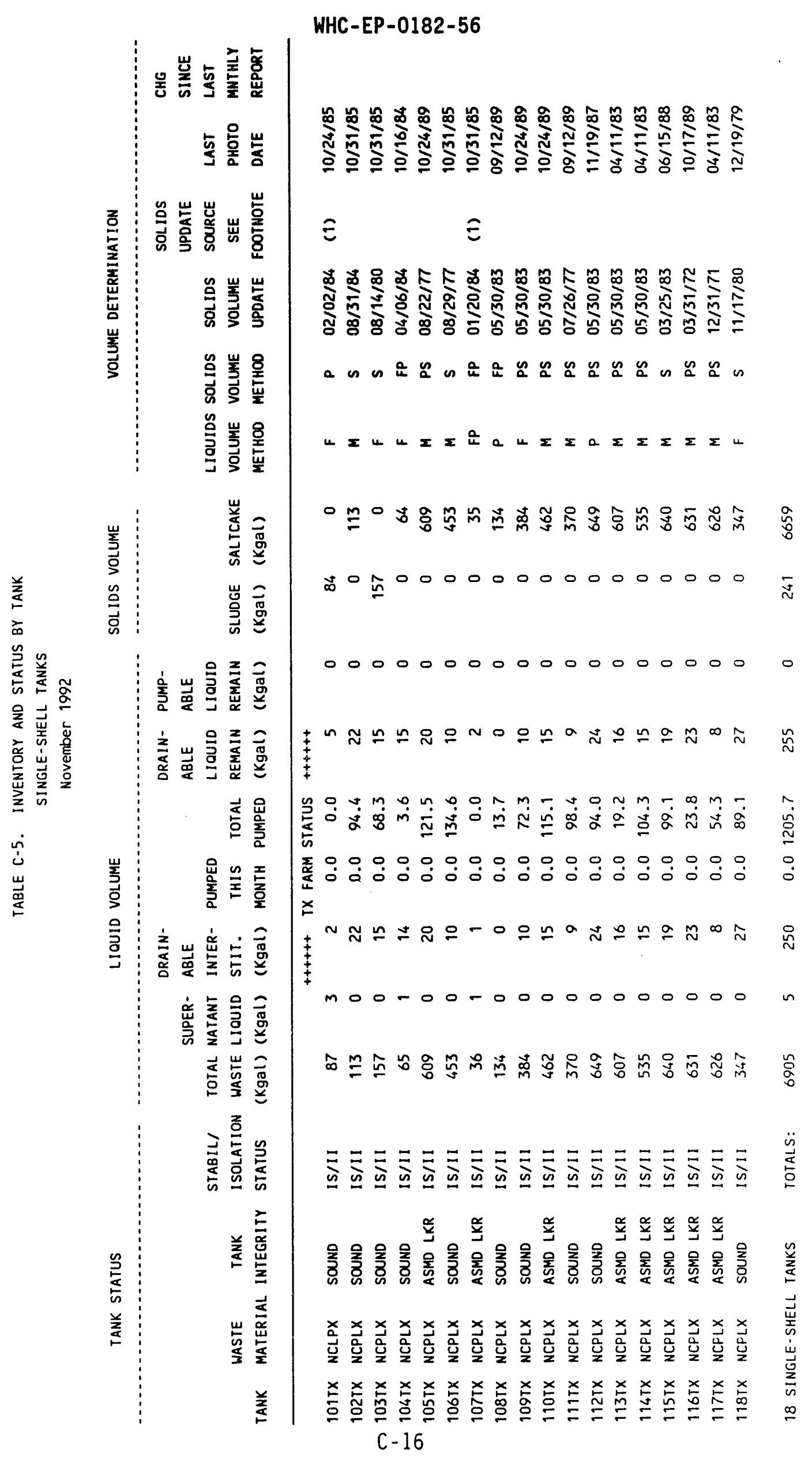




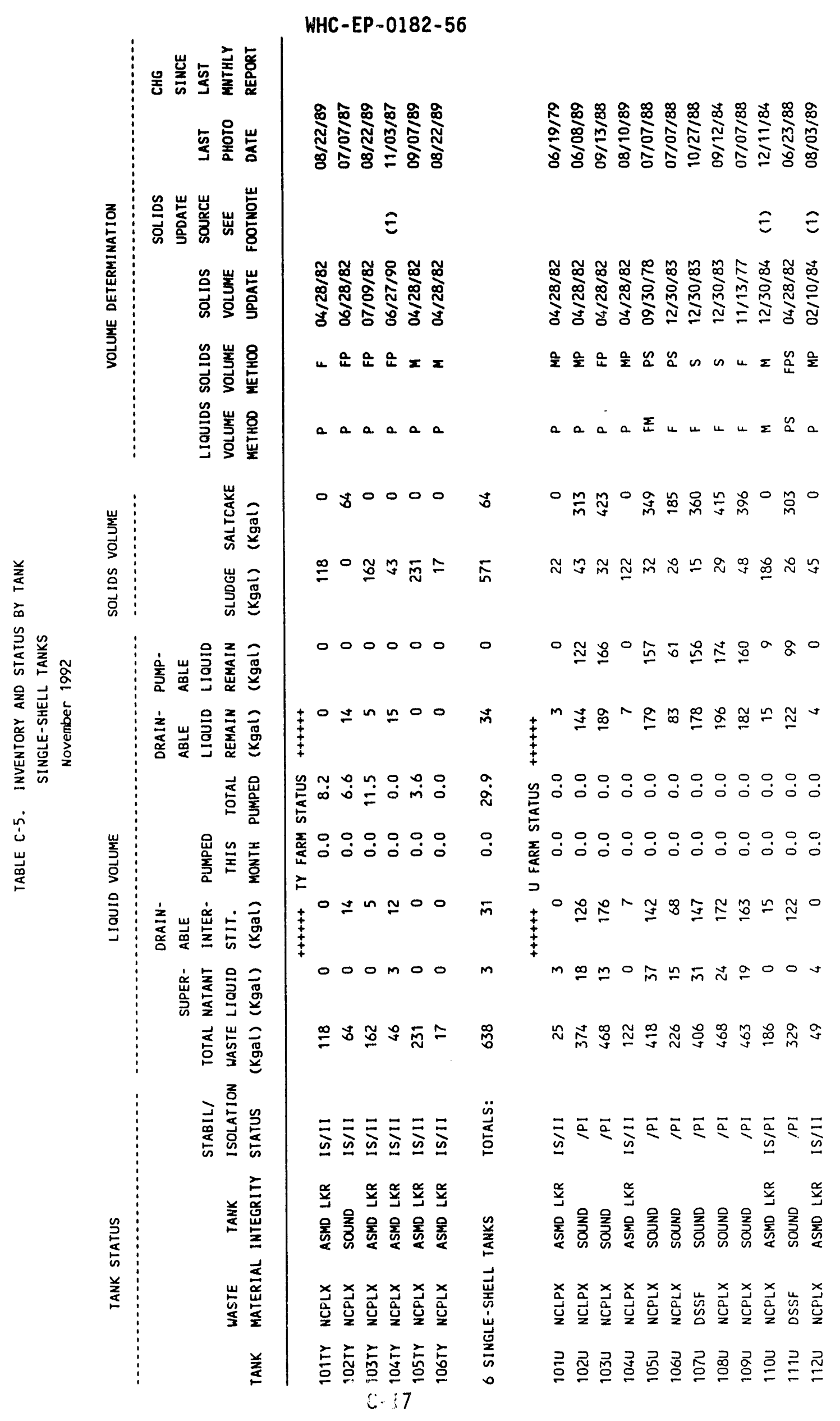




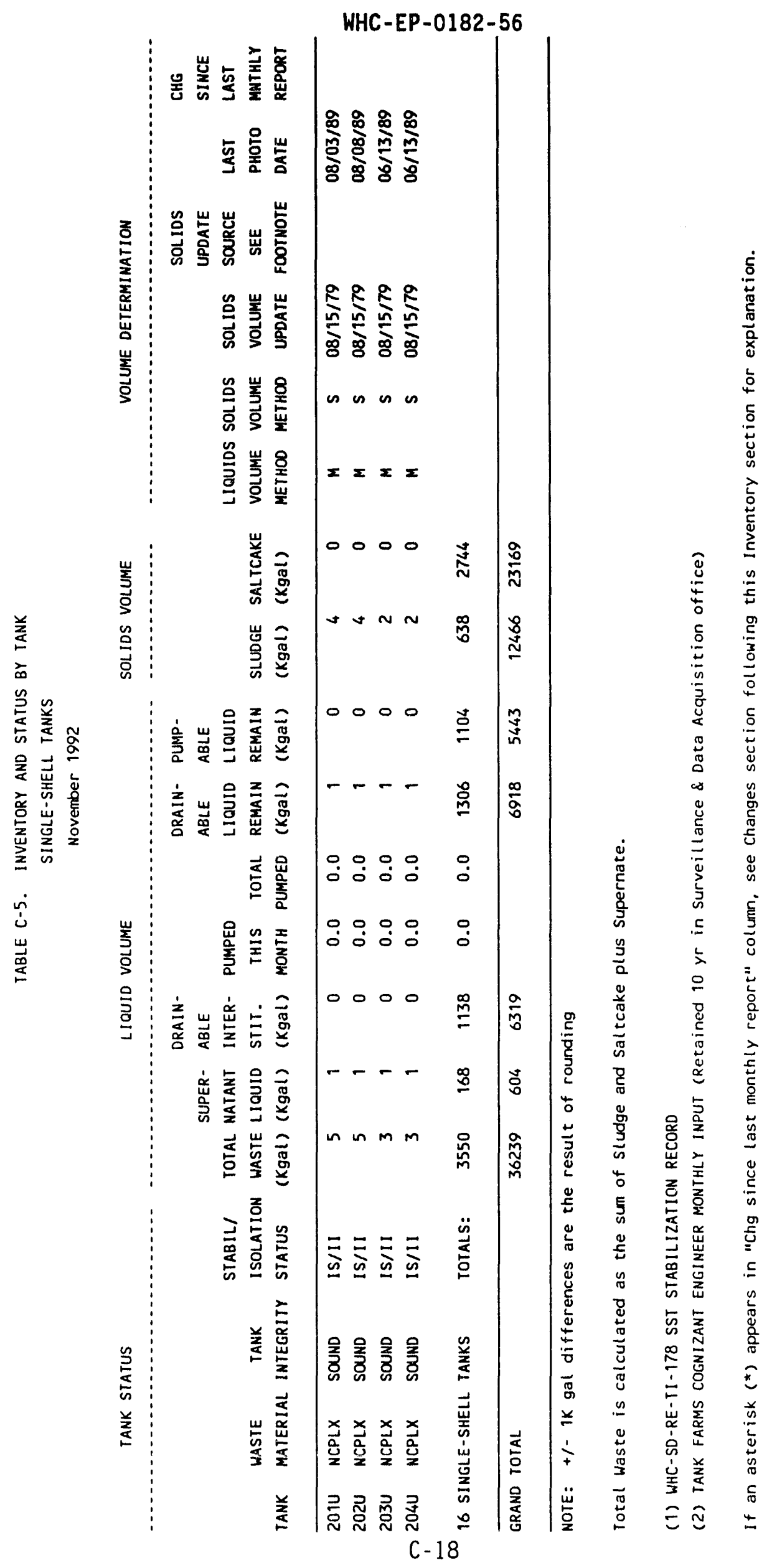


WHC-EP-0182-56

\section{APPENDIX D}

\section{PERFORMANCE SUMMARY}


WHC-EP-0182-56

This page intentionally left blank.

D-2 


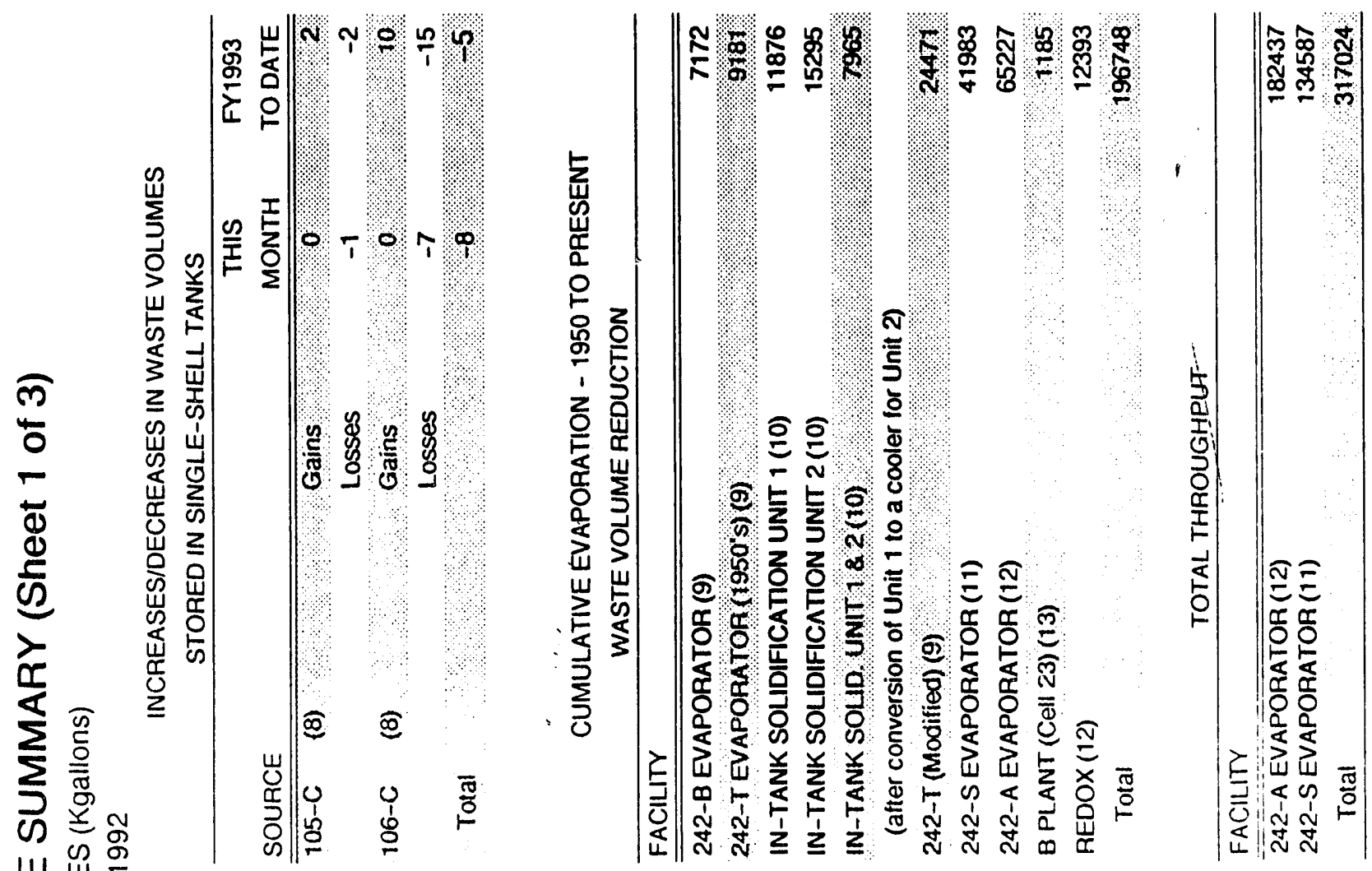

山出

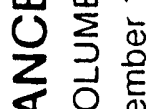

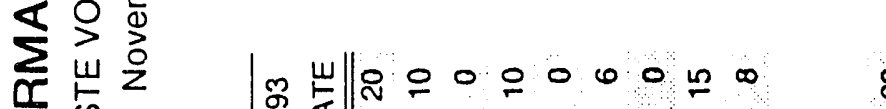

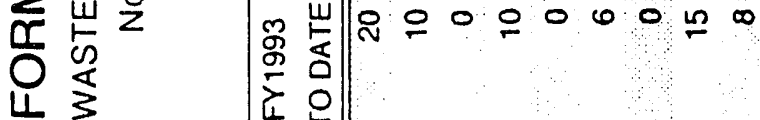

8

के

$\circ \circ \pi$

岂 岂

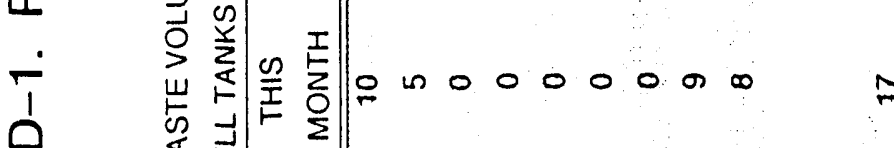

$\infty 0$ nN $N 00$ T

山

$\gamma$

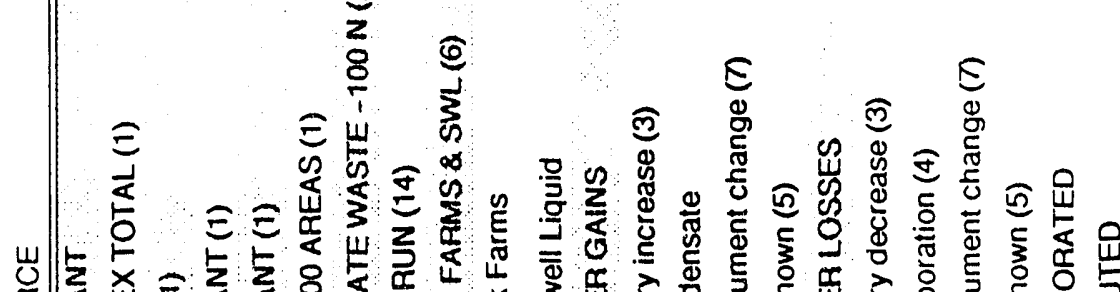

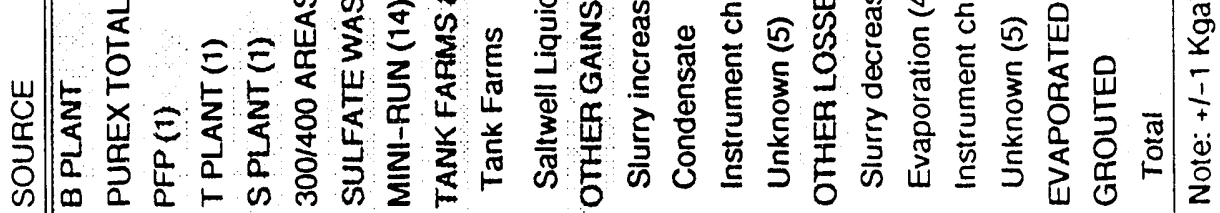

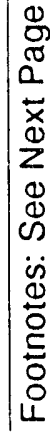


Footnotes:

\section{INCREASES/DECREASES IN WASTE VOLUMES}

(1) Including Flush

(2) Sulfate waste is generated from ion exchange backflushing and sand filter clean out, resulting in sulfate waste $\left(\mathrm{Na}_{2} \mathrm{SO}_{4}\right)$.

(3) Slurry increase/growth is caused by gas generation within the waste. The gas which is trapped in the waste expands in the tank causing the surface level and volume to increase. Slurry decrease results from the periodic release of gas in the waste.

(4) Aging waste tanks

(5) Unknown waste gains or losses may be the result of rounding calculations, clean water slowly leaking through a valve, changes in levels (expansion/constraction) because of to ambient temperature changes, different measuring devices being used by Tank Farm operators, transfers taking place during the end of the month, Tank Farm activities such as miscellaneous water additions not associated with facility waste generation, or the addition of water which is added to aging waste tanks and then evaporated off.

(6) Includes Tank Farms miscellaneous flushes (flushes are used to "clean out" pipelines and reduce personnel exposure, reduce potential for waste incompatibility, prevent line plugging, and reduce waste content of potential spills or leaks), and saltwell liquid, which results from pumping of single-shell tanks to double-shell tanks.

(7) Liquid level measurement instrument changes from the automatic FIC to manual tape (and vice versa) result in unusual gains or losses because the manual tape may rest on an uneven crust surface giving a different reading from that of the automatic FIC. These instrument changes are made when the automatic FIC is out of service and the reading from the manual tape is used for reporting purposes. The reported reading reverts back to the automatic FIC when it is repaired.

(8) Water is periodically added to $105-C$ and $106-C$ to provide evaporative cooling. Losses due to evaporation are calculated assuming all losses are evaporative losses. Drywell monitoring for leak detection is done monthly on tank 105-C. Some drywells are monitored weekly and some are monitored every two weeks on tank 106-C. If there are any indications of a leak from these tanks, the assumption that all losses are due to evaporation will be reevaluated. 
TABLE D-1. Performance Summary (Sheet 3 of 3 )

\section{WASTE VOLUME REDUCTION}

(9) Currently inoperative. These evaporator systems (242-B and 242-T) were installed in 1952 in each of the two operating areas to remove water from the waste, and ran for approximately 4 yr after which both units were shut down. The 242-T Evaporator was reactivated in December 1965, and shut down again in April 1976.

(10) Currently inoperative. These two in-tank solidification (ITS) units provided in-tank heating to promote in-tank boiling or evaporation. The ITS Unit 1 started up March 1965, and ITS Unit 2 started up February 1968. In August 1971, ITS Unit 1 was converted from an evaporator to a cooler for ITS Unit 2. Both units were shut down June 1974.

(11) Currently inoperative. The 242-S Evaporator-Crystallizer was started up November 1973, and shut down March 1980, when its processing campaign was completed. It is in standby mode with no future mission. This evaporator operates under a vacuum, employing evaporative concentration with subsequent crystallization and precipitation of salt crystals.

(12) Currently inoperative. The 242-A Evaporator-Crystallizer was started up March 1977, and shut down April 1989 because of regulatory issues, and has remained shut down for subsequent upgrading. The restart schedule specifies January 4, 1993, as the projected start-up date. This evaporator operates under a vacuum, employing evaporative concentration with subsequent crystallization and precipitation of salt crystals (forming saltcake).

(13) Currently inoperative. Additional concentration of wastes was completed by using the concentrators at REDOX and B Plant. The REDOX concentrator was used from July 1967 to June 1972, while the B Plant concentrator was used from July 1967 to February 1968.

(14) Waste generated for training and testing purposes prior to Evaporator restart. 
WHC-EP-0182-56

This page intentionally left blank

D-6 
WHC-EP-0182-56

\section{APPENDIX E}

\section{LIQUID STATUS AND PUMPABLE LIQUID}

REMAINING IN TANKS 
WHC-EP-0182-56

This page intentionally left blank.

$E-2$ 
WHC-EP-0182-56

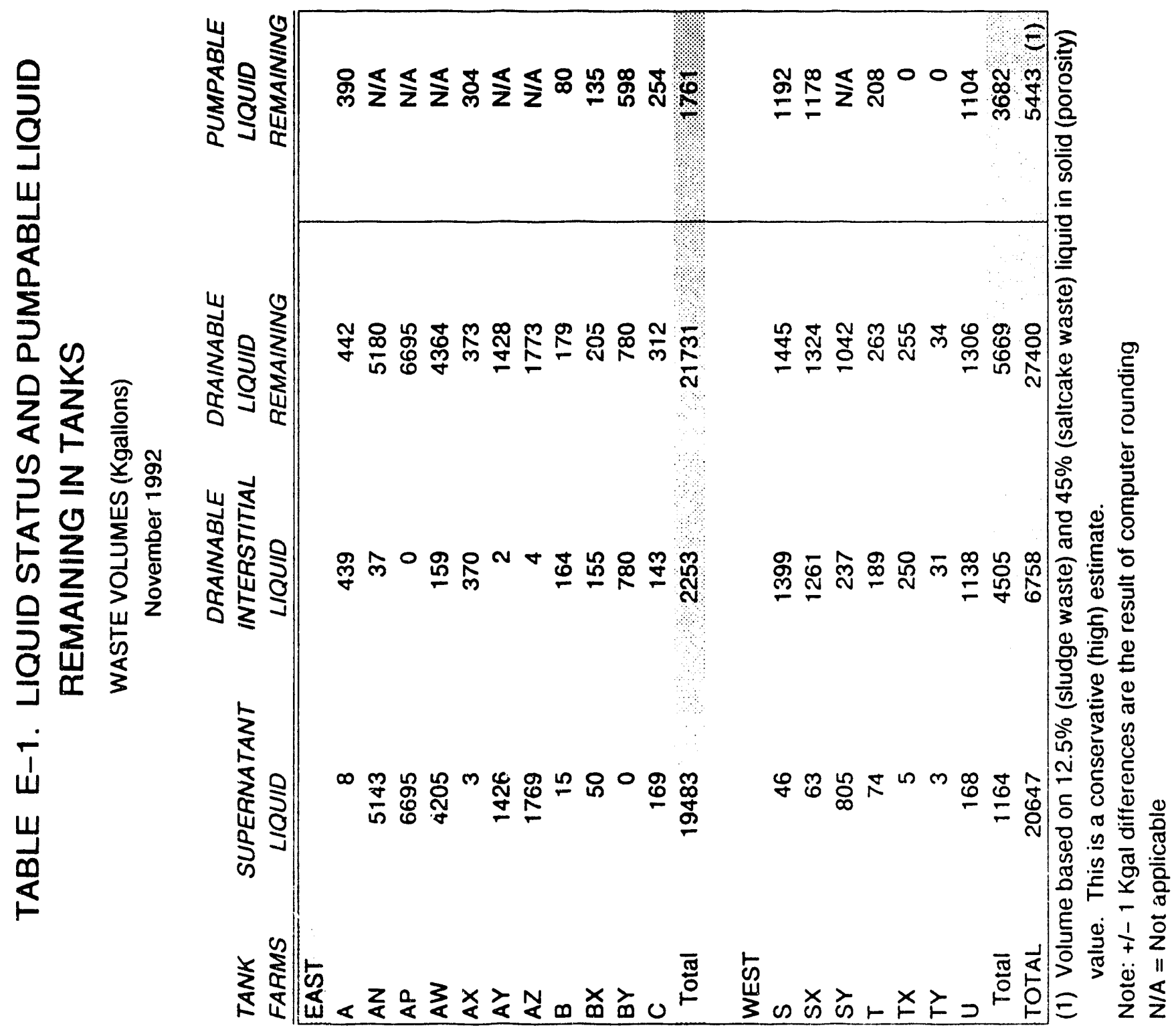


WHC-EP-0182-56

This page intentionally left blank. 
WHC-EP-0182-56

\section{APPENDIX F}

PUMPING RECORD 
WHC-EP-0182-56

This page intentionally left blank. 


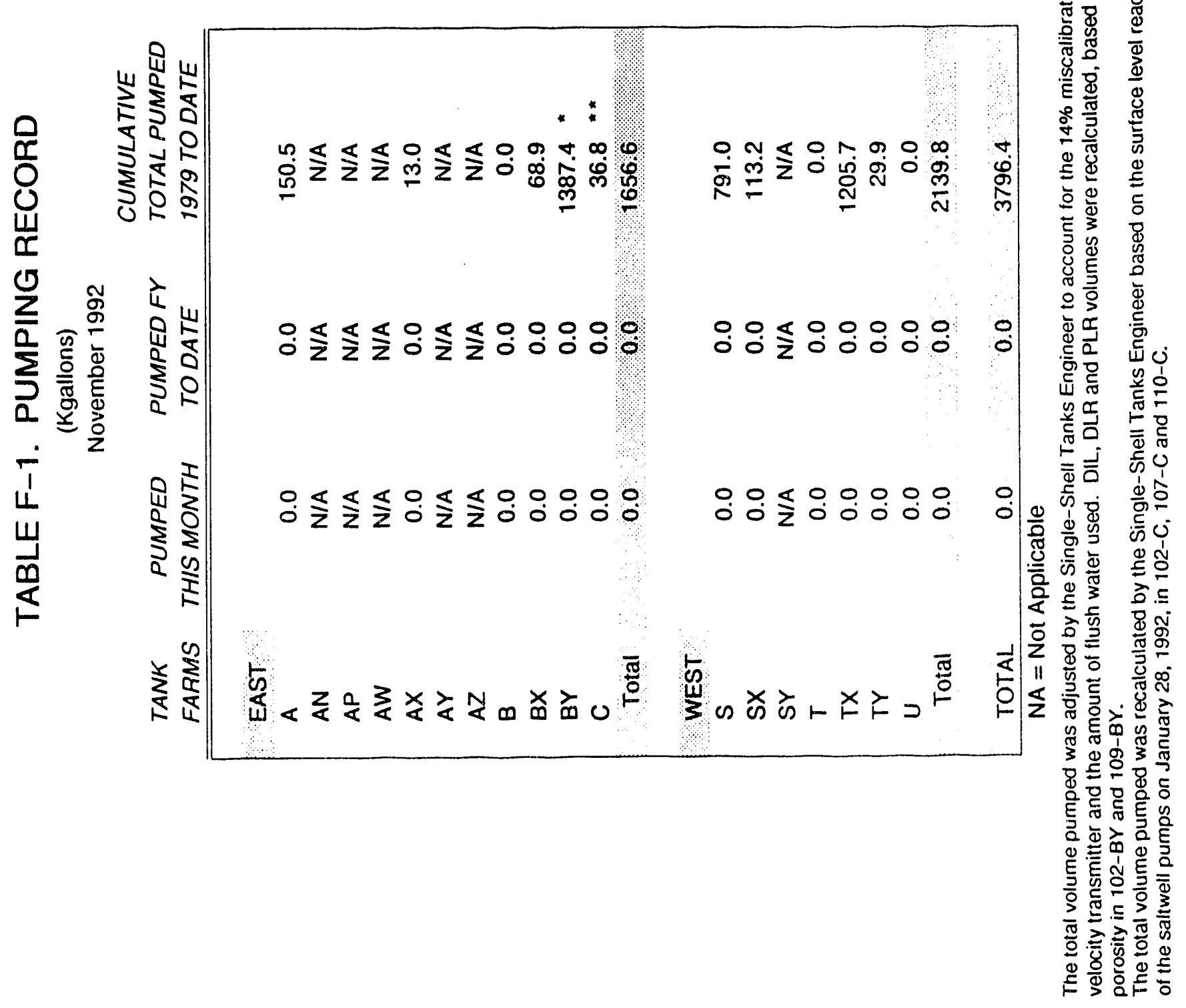


WHC-EP-0182 .56

This page intentionally left blank.

F-4 
WHC-EP-0182-56

\section{APPENDIX G}

CATCH TANKS AND SPECIAL SURVEILLANCE FACILITIES 
WHC-EP-0182-56

This page intentionally left blank.

G-2 

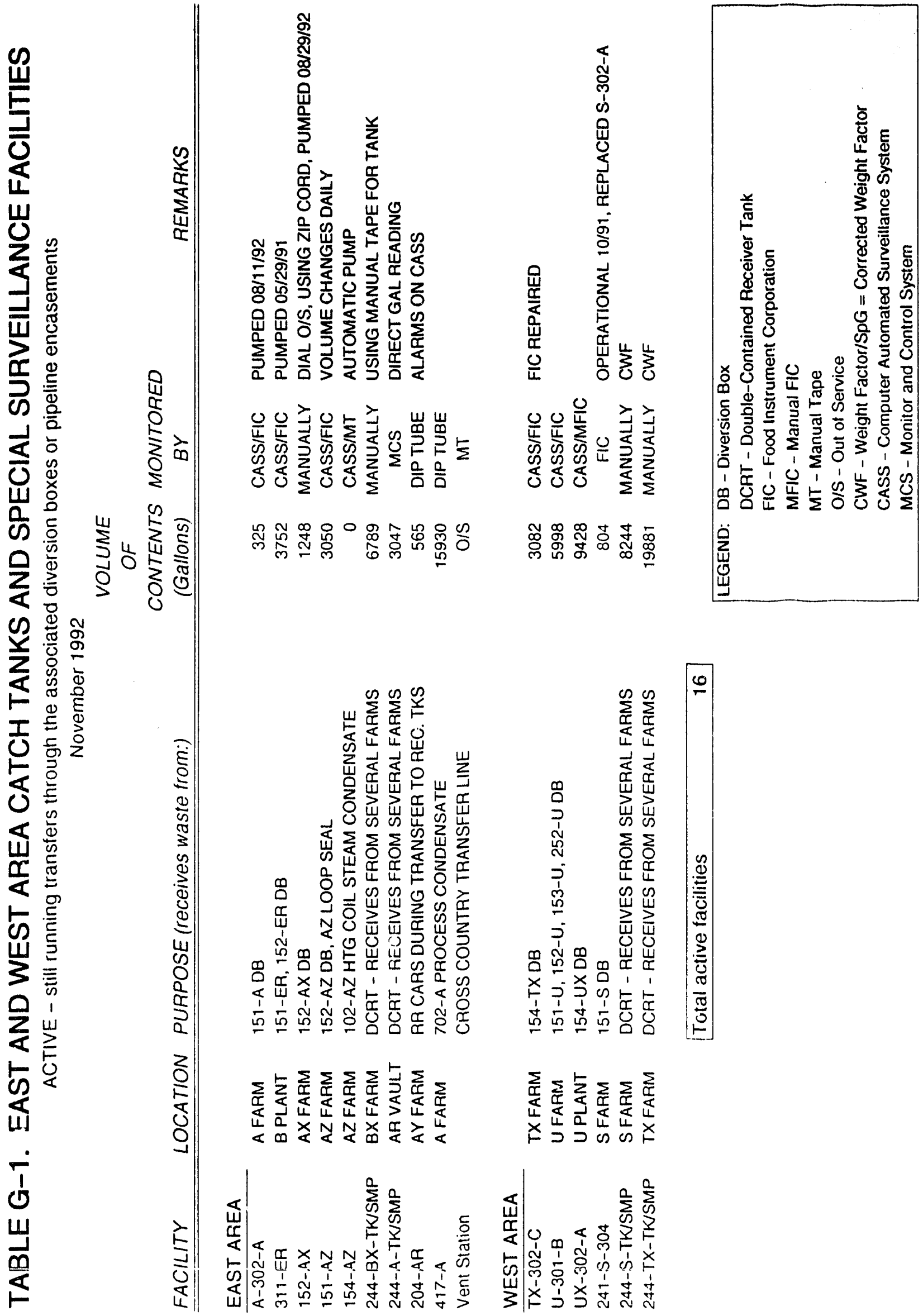

का山

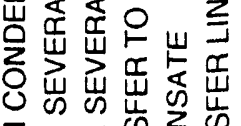

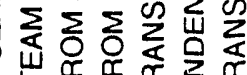

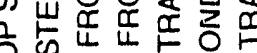

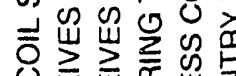

嵌岕岁

莡

$0 \pi \times N=1$ 的

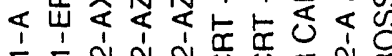

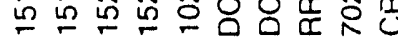

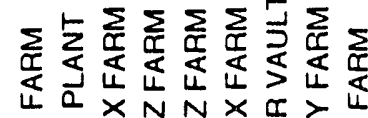

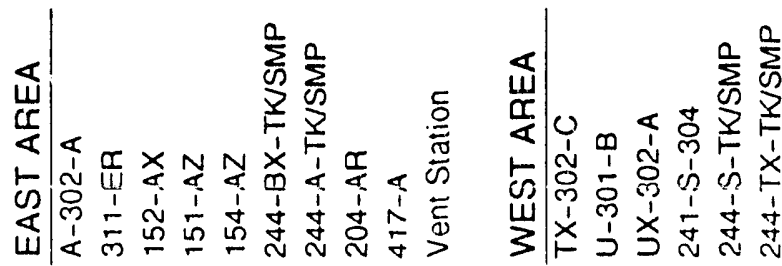


WHC-EP-0182-56

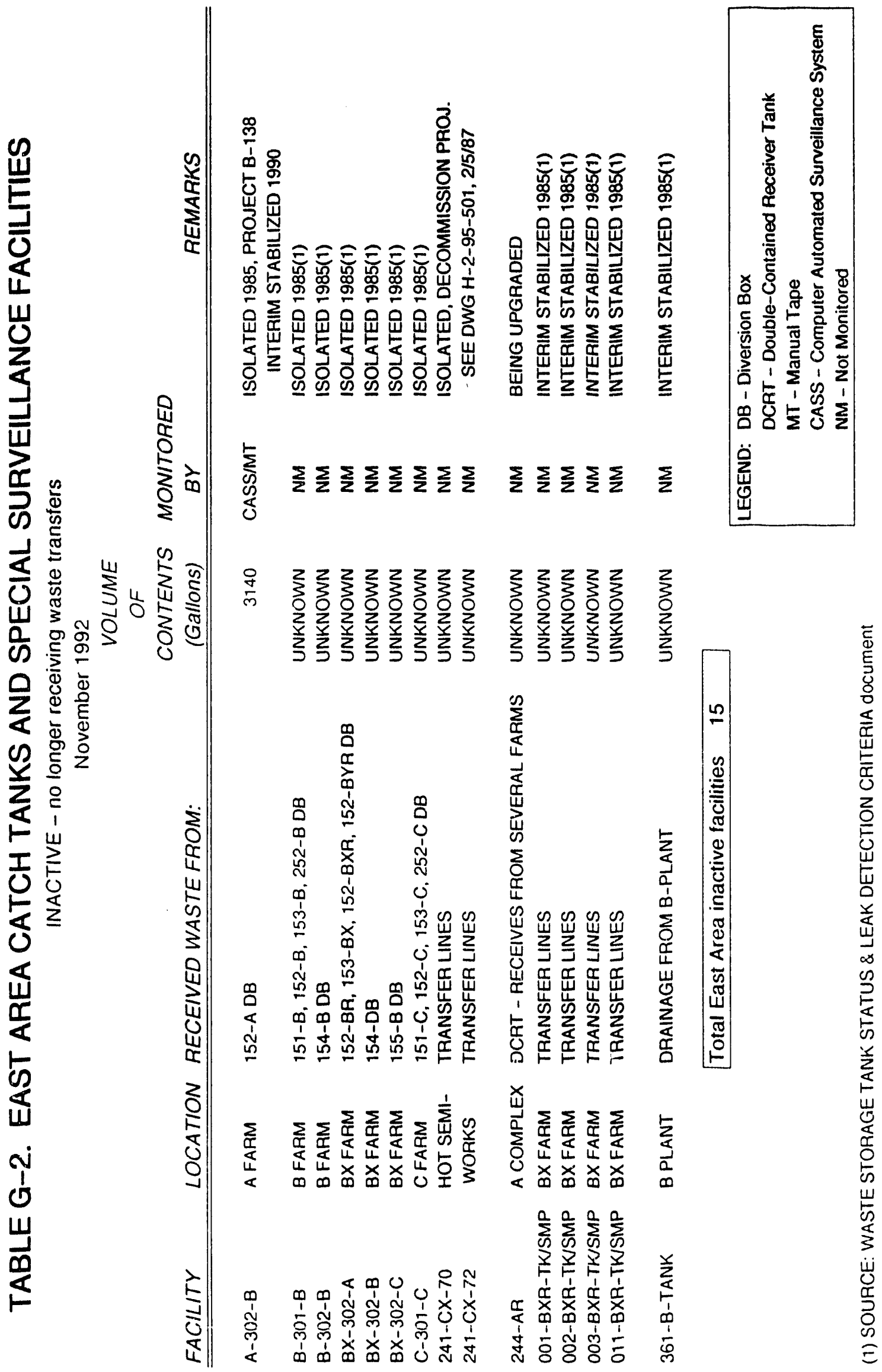




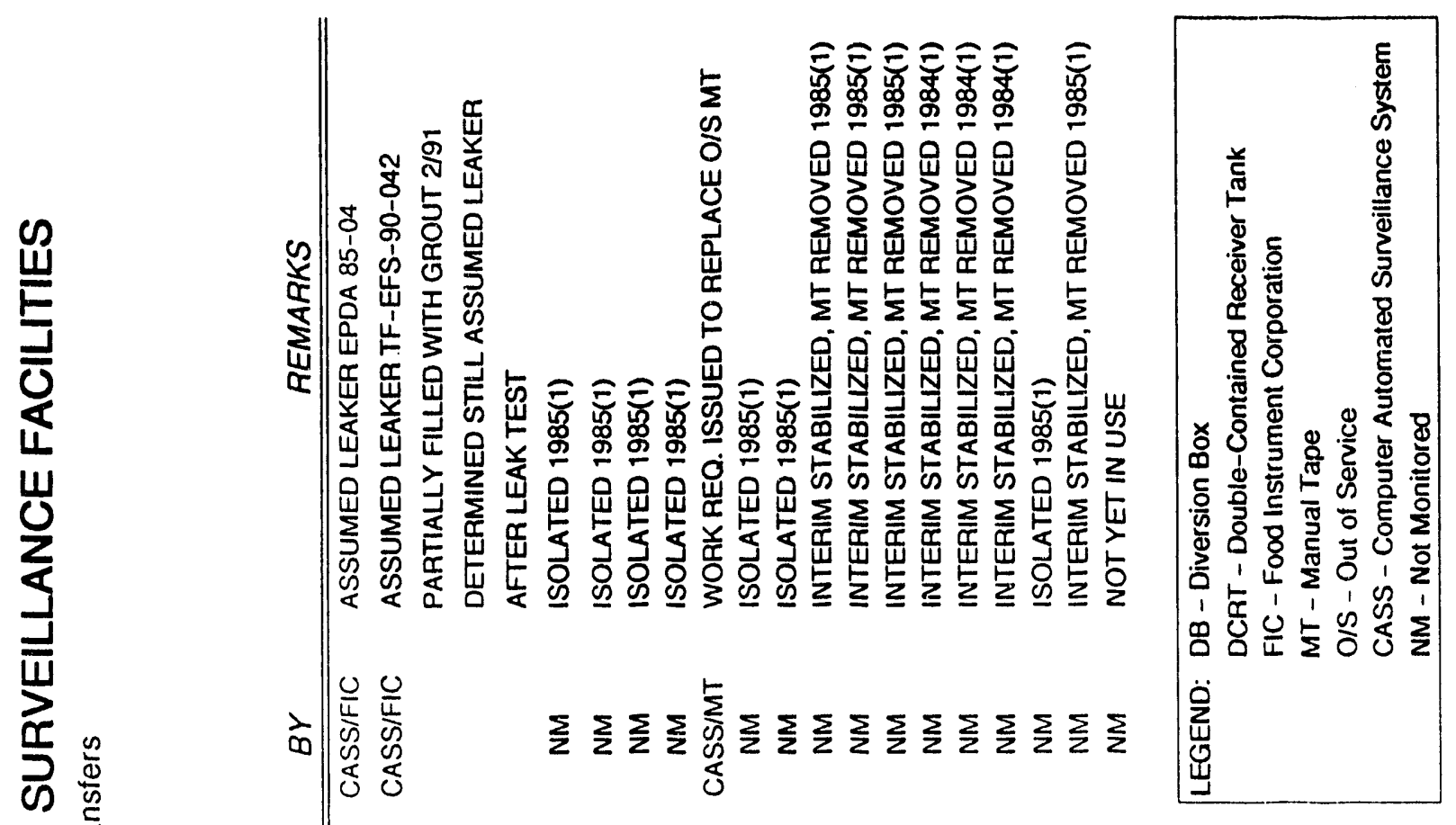

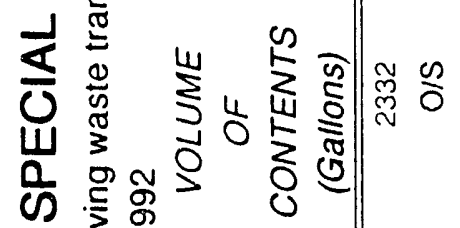

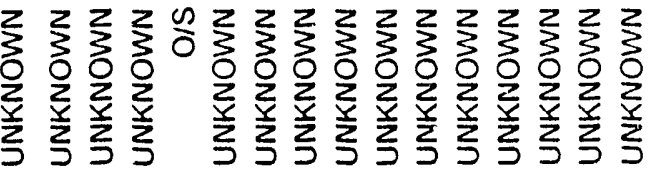

ง

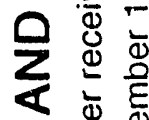

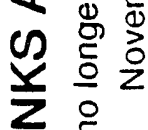

\&

沗

$\dot{p}$
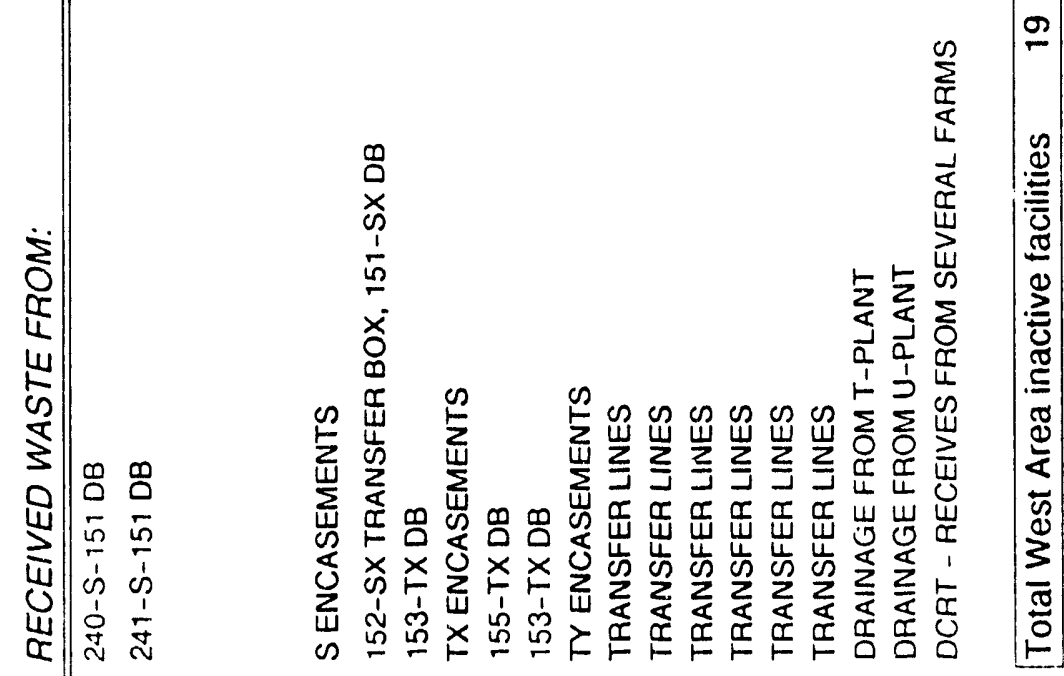

5

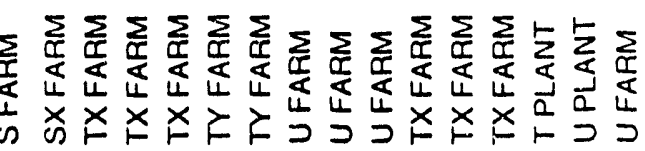

1

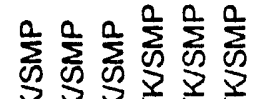

ชิ 
WHC-EP-0182-56

This page intentionally left blank. 
WHC-EP-0182-56

APPENDIX H

\section{LEAK VOLUME ESTIMATES}

$\mathrm{H}-1$ 
WHC-EP-0182-56

This page intentionally left blank. 
WHC-EP-0182-56

TABLE H-1. SINGLE-SHELL TANK LEAK VOLUME ESTIMATES (4) (Sheet 1 of 5)

Date Declared

Confirmed or

Associated

Interim

Tank No.

Assumed Leaker (3)

Volume (2)

Curies

Stabilized

$241-A-103$

$241-A-104$
$241-A-105$

(1)

1987
1975
1963

(Gallons)

137 cs

Date

241-AX-10

241-AX-104

241-B-101

$241-B-103$
$241-B-105$

241-B-107

241-B-110

241-B-111

241-B-112

$241-\mathrm{B}-203$

241-B-204

241-BX-101

241-BX-102

$241-B X-108$

$241-B X-110$

$\frac{241-B X-111 \text { (i) }}{241-B Y-103}$

$241-B Y-105$

241-BY-106

$241-\mathrm{BY}-107$
$241-\mathrm{BY}-108$

241-C-101

241-C- 110

241-C-201

241-C-202

$241-\mathrm{C}-203$
$241-\mathrm{C}-204$

241-S-104

241-SX-104

241-SX-108

$241-S X-109$ (6)
$241-S X-110$

$\frac{241-5 X-110}{241-S X-111}$

241-SX-112

241-SX-113

241-SX-114

241-SX-115

241-T-101

241-T-103

241-T-106

241-T-107

241-T-108

241-T-109

241-T-111

241-TX-105

$241-T X-107$

241-TX-113

241-TX-114

241-TX-115

241-TX-116

241-TX-117

$241-T Y-101$

241-TY-103

241-TY-104

241-TY-105

241-TY-106

241-U-101

$241-U-104$
$241-U-110$

241-U-112.

67 Tanks

$\begin{array}{cccc}10000 \text { to } & 85000 \text { to } \\ 277000 & 760000 & \text { (b) } \\ 1988 & 3000(10) & \end{array}$

$\begin{array}{ll}8 / 88 & 1987 \\ 9 / 78 & 1983 \\ 7 / 79 & 1991\end{array}$

Leak Estimate Updated Reference

-

1977
1974
1978

1974
1978
1978

1978

1981

1978

1978

1983

1984

1972
1971
1974

1974

1984

1973

1984
1984

1984

$3000(10)$

$\overline{--}(8)$

-- (8)

$10000\left(\begin{array}{l}10 \\ 10\end{array}\right.$

$200 \overline{0}$

1200
300
400

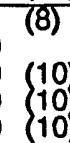

$400(10)$

$7000 \overline{-1}$

2500

-- (8)

$<5000$

1972

1980

1968

(5) 1988

(5) $\quad 1988$

$=-(8)$

15100 (10) $<5000$

20000 (10)

2000
5500
550

1968

450

400
350

(10)

$24000(10)$

1988
1964

$6000(10)$

(b) $\quad 1962$

N/A = not applicable (not yet interim stabilized)

FOOTNOTES: SEE NEXT PAGE

$\begin{array}{lll}9 / 88 & 1989 & (\text { h } \\ 8 / 81 & 1989 & (g)\end{array}$

$\begin{array}{lll}3 / 81 & 1989 & \text { (g) } \\ 2 / 85 & 1989 & \text { g) } \\ 12 / 84 & 1989 & \text { (d) } \\ 3 / 85 & 1986 & \text { (d) }\end{array}$

$12 / 84 \quad 1986$

$6 / 85-1989$

$6 / 85$
$5 / 85$

(a)

(b)

)

(i)




\section{WHC-EP-0182-56}

TABLE H-1. Single-Shell Tank Leak Volume Estimates

(Sheet 2 of 5 )

\section{Footnotes:}

(1) Current estimates (see reference b) are that $610 \mathrm{Kgal}$ of cooling water was added to Tank 241-A-105 from November 1970 to December 1978 to aid in evaporative cooling. In accordance with Dangerous Waste Regulations (Washington Administrative Code 173-303-070 (2)(a)( $i$ i), as amended, Washington State Department of Ecology, 1990, 01ympia, Washington), any of this cooling water that has been added and subsequently leaked from the tank must be classified as a waste and should be included in the total leak volume. In August 1991, the leak volume estimate for this tank was updated and moved into compliance with the WAC regulations. Previous estimates excluded the cooling water leaks from the total leak volume estimates because the waste content (concentration) in the cooling water which leaked should be much less than the original liquid waste in the tank (the sludge is relatively insoluble). The total leak volume estimate in this report (10 Kgal to $277 \mathrm{Kgal}$ ) is based on the following (see References).

1. Reference (b) contains an estimate of $5 \mathrm{Kgal}$ to $15 \mathrm{Kgal}$ for the initial leak prior to August 1968.

2. Reference (b) contains an estimate of $5 \mathrm{kgal}$ to $30 \mathrm{Kgal}$ for the leak while the tank was being sluiced from August 1968 to November 1970.

3. Reference (b) contains an estimate of $610 \mathrm{Kgal}$ of cooling water added to the tank from November 1970 to December 1978 but it was estimated that the leakage was small during this period. This reference contains the statement "Sufficient heat was generated in the tank to evaporate most, and perhaps nearly all, of this water." This results in a low estimate of zero gallons leakage from November 1970 to December 1978.

4. Reference (c) contains an estimate that 378 to $410 \mathrm{Kgal}$ evaporated out of the tank from November 1970 to December 1978. Subtracting the minimum evaporation estimate from the cooling water added estimate provides a range from 0 to $232 \mathrm{Kgal}$ of cooling water leakage from November 1970 to December 1978.

\section{Low Estimate High Estimate}

Prior to August 1968

August 1968 to November 1970

November 1970 to December 1978 Totals

$$
\begin{array}{rr}
5,000 & 15,000 \\
5,000 & 30,000 \\
\hline 0 & 232,000 \\
\hline 10,000 & 277,000
\end{array}
$$

(2) These leak volume estimates do not include (with some exceptions), such things as: (a) cooling/raw water leaks, (b) intrusions (rain infiltration) and subsequent leaks, (c) leaks inside the tank farm but not through the tank liner (surface leaks, pipeline leaks, leaks at the joint for the overflow or fill lines, etc.), and (d) leaks from catch tanks, diversion boxes, encasements, etc. 
TABLE H-1. Single-Shell Tank Leak Volume Estimates

(Sheet 3 of 5 )

(3) In many cases, a leak was suspected long before it was identified or confirmed. In 1984, the criteria designations of "suspected leaker," "questionable integrity," "confirmed leaker," "declared leaker, " "borderline," and "dormant," were merged into one category now reported as "assumed leaker." See reference (f) for explanation of when, how long, and how fast some of the tanks leaked.

(4) There is an effort currently in progress to reevaluate these leak volume estimates.

(5) The leak volume estimate date for these tanks is before the "declared leaker" date because the tank was in a "suspected leaker" or "questionable integrity" status; however, a leak volume had been estimated prior to the tank being reclassified.

(6) The increasing radiation levels in drywells and laterals associated with these three tanks could be indicative of a continuing leak or movement of existing radionuclides in the soil. There is no conclusive way to confirm these observations.

(7) These four tanks also show slight indications of continuing leaks or movement of radionuclides in the soil.

(8) Methods were used to estimate the leak volumes from these 19 tanks based on the assumption that their cumulative leakage is approximately the same as for 18 of the 24 tanks identified in footnote (10). For more details see reference $(\mathrm{g})$. The total leak volume estimate for these tanks is $150 \mathrm{Kgal}$ (rounded to the nearest $10 \mathrm{Kgal}$ ).

(9) The total has been rounded to the nearest $50 \mathrm{Kgal}$. Upperbound values were used in many cases in developing these estimates. It is likely that some of these tanks have not actually leaked.

(10) Leak volume estimate is based solely on observed liquid level decreases in these tanks. This is considered to be the most accurate method for estimating leak volumes. 
TABLE H-1. Single-Shell Tank Leak Volume Estimates. (Sheet 4 of 5 )

References:

(a) Murthy, K.S., et al, June 1983, Assessment of Single-Shell Tank Residual Liquid Issues at Hanford Site, Washington, PNL-4688, Pacific Northwest Laboratory, Richland, Washington.

(b) WHC, 1991a, Tank 241-A-105 Leak Assessment, WHC-MR-0264, Westinghouse Hanford Company, Richland, Washington.

(c) WHC, 1991b, Tank 241-A-105 Evaporation Estimate 1970 Through 1978, WHC-EP-0410, Westinghouse Hanford Company, Richland, Washington.

(d) Smith, D. A., January 1986, Single-Shell Tank Isolation Safety Analysis Report, SD-WM-SAR-006, Rev. 1, Westinghouse Hanford Company, Richland, Washington.

(e) McCann, D. C., and T. S. Vail, September 1984, Waste Status Summary, RHO-RE-SR-14, Rockwell Hanford Operations, Richland, Washington.

(f) Catlin, R. J., March 1980, Assessment of the Surveillance Program of the High-Level Waste Storage Tanks at Hanford, Hanford Engineering Development Laboratory, Richland, Washington.

(g) Baumhardt, R. J., May 15, 1989, Letter to R. E. Gerton, U.S. Department of Energy-Richland Operations Office, Single-Shell Tank Leak Volumes, Westinghouse Hanford Company, Richland, Washington.

(h) WHC, 1990, Occurrence Report "Surface Level Measurement Decrease in Single-She17 Tank 241-AX-102," WHC-U0-89-023-TF-05, Westinghouse Hanford Compary, Richland, Washington.

(i) Groth, D. R., July 1, 1987, Internal Memorandum to R. J. Baumhardt, Liquid Level Losses in Tanks 241-C-201, -202 and -204, 65950-87-517, Westinghouse Hanford Company, Richland, Washington.

(j) Groth, D. R. and G. C. Owens, May 15, 1987, Internal Memorandum to J. H. Roecker, Tank 103-A Integrity Evaluation, Westinghouse Hanford Company, Richland, Washington.

(k) Campbe11, G. D., July 8, 1988, Internal Memorandum to R. K. Welty, Engineering Investigation: Interstitial Liquid Level Decrease in Tank 241-SX-104, 13331-88-416, Westinghouse Hanford Company, Richland, Washington.

(1) ERDA, 1975, Final Environmental Statement Waste Management Operations, Hanford Reservation, Richland, Washington, ERDA-1538, 2 vols., U.S. Energy Research and Development Administration, Washington, D.C.

(m) WHC, 1992a, Tank 241-SX-108 Leak Assessment, WHC-MR-0300, Westinghouse Hanford Company, Richland, Washington.

(n) WHC, 1992b, Tank 241-SX-109 Leak Assessment, WHC-MR-0301, West inghouse Hanford Company, Richland, Washington. 


\section{WHC-EP-0182-56}

TABLE H-1. Single-Shell Tank Leak Volume Estimates.

(Sheet 5 of 5 )

(0) WHC, 1992C, Tank 241-SX-115 Leak Assessment, WHC-MR-0302, Westinghouse Hanford Company, Richland, Washington.

(p) WHC-1992d, Occurrence Report, "Apparent Decrease in Liquid Level in Single Shel1 Underground Storage Tank 241-T-101, Leak Suspected; Investigation Continuing," RL-WHC-TANKFARM-1992-0073, Westinghouse Hanford Company, Richland, Washington. 
WHC-EP-0182-56

This page intentionally left blank. 


\section{DISTRIBUTION}

\section{Number of copies}

\section{OFFSITE - USA}

20

U. S. Department of Energy-Headquarters 1000 Independence Avenue, SW Washington, D. C. 20585
Emile Bernard
H. Calley
H. Eckart
Teresa Fryberger
Sherry Gibson
A. Griffith
L. Gunn
D. Gupta
J. S. Kang
Kenneth Lang
J. C. Lehr
G. Mellinger
John Morrey
C. 0'Dell
C. Pepson
J. C. Tseng
S. Woodbury

$\begin{array}{ll}E M-50 & \text { TREV II } \\ \text { EM-36 } & \text { TREV I I } \\ \text { EM-36 } & \text { TREV I } \\ \text { EM-542 } & \text { TREV I I } \\ \text { EM-55 } & \text { TREV I I } \\ \text { EM-322 } & \text { TREV I } / 343 \\ \text { EM-36 } & \text { TREV I I } \\ \text { EM-36 } & \text { TREV I I } \\ \text { EM-351 } & \text { TREV I } / 368 \\ \text { EM-36 } & \text { TREV I I } \\ \text { EM-442 } & \text { TREV I } / 160 \\ \text { EM-36 } & \text { TREV I I } \\ \text { NW Labs) } & \text { TREV I } \\ \text { EM-36 } & \text { TREV II } \\ \text { EM-36 } & \text { TREV I I } \\ \text { EM-36 } & \text { TREV I } / 366 \\ \text { EH-222 } & \text { FORS } / 3 G-092\end{array}$

19901 Germantown Rd, Germantown, MD 20585
R. Lasky
$\mathrm{EH}-32.1$
GTN/G-115
J. Psaras
NS - 20 GTN
P. Worthington
NS-20 GTN

U. S. Department of Energy - Oak Ridge Operations Office P. 0. Box 2001

Oak Ridge, TN 37831
W. D. Adams
EW-40

7

U. S. Department of Energy - Savannah River Site

P. 0. Box A

Aiken, SC 29808
C. Anderson
$707-\mathrm{H}$
Michael Chandler
$703-\mathrm{H}$
Mazen Shurrab
$704-\mathrm{H}$
T. C. Temple
L. Sjostrom
W. R. West
V. Wheeler
704-8H
704-S 
WHC-EP-0182-56

Distribution - continued

U. S. Department of Energy - Idaho Operations

785 D. 0. E. Place

Idaho Falls, ID 83402

C. Enos

MS -1139

D. Engelman

MS -1118

G. Woodall

MS-1139

U. S. Environmental Protection Agency Region 10

712 Swift Boulevard, Suite 5

Richland, WA 99352

P. T. Day

Washington State Department of Ecology Nuclear \& Mixed Waste Management

719 Sleater-Kinney Road S. E., Suite 200 Lacey, WA 98503-1138

R. Stanley

M. T. Gordon

7601 W. Clearwater \#102

Kennewick, WA 99336

Greta Davis

Washington State Department of Health

Radiation Protection Section

Industrial Park Building 5, LE-13

01 ympia, WA 98504

A. Conklin

Oregon State Department of Energy

625 Marion St. N.E.

Salem, OR 97310

Janet Franco

1

Oregon State Water Resources Department

Ground Water Hanford Studies

3850 Portl and Road

Salem, OR 97310

R. 0. Patt

Lawrence Livermore National Laboratory

Box 808, East Avenue

Livermore, CA 94550

B. C. Hudson 
WHC-EP-0182-56

\section{Distribution - continued}

Oak Ridge National Laboratory

P. 0. Box 2009

Oak Ridge, TN 37831-6385

C. Forsberg

MS -6495

T. S. Kress

MS -8088

Bldg 9108

Chemical Technology Division

P. 0. Box 2008

Emory D. Collins

5

Los Alamos National Laboratory

P. 0. Box 1663

Los Alamos, NM 87545

Stephen Agnew

Group INC-14

C -346

T. Larson

C- 915

Sylvia Lee

K-557

A. Nuels

$\mathrm{N}-6$

H. Sullivan

N-6

3

Brookhaven National Laboratory

50 Rutherford

Upton, NY 11973

K. K. Bandyopadhyay, B1dg 475-C

M. Reich, Bldg 475-C

P. D. Kaib, Bidg. 703

1

Brookhaven National Laboratory

1409 Jan Drive

Wilmington, DE 19803

Michael Streicher

2

Sandia National Laboratories

1515 Eubank, NE

P. 0. Box 5800

Albuquerque, NM 87185

Scott Slezak, Division 6402

Leon D. Chapman, Program Manager

Industrial Waste Reduction Program

2

Massachusetts Institute of Technology

77 Massachusetts Avenue

Cambridge, MA 02139

Mujid S. Kazimi

Professor and Head

Department of Nuclear Engineering

$$
\text { Distr-3 }
$$


WHC-EP-0182-56

\section{Distribution - continued}

2

BDM International. Inc.

20030 Century B1vd, Suite 101

Germantown, MD 20874

P. Kiang

K. J. Mahoney

2

SAIC

Trevion 1, Suite 300

12850 Middlebrook Rd.

Germantown, MD 20874

J. Bunting

J. R. Pearring

1

102 Windham Road

Oak Ridge, TN 37830

D. 0. Campbel1

1

SAIC

$\frac{1845}{184}$ Terminal Drive, Suite 130

Richland, WA 99352

J. Mishima

1

Harvard School of Public Health

665 Huntington Avenue

Boston, MA 02115

M. First

1

Confederated Tribes, Umatilla Indian Reservation

P. 0. Box 638

Pendleton, OR 97801

Rick George

4

West Valley Nuclear Services Co.

P. 0. Box 191

West Valley, NY 14171

S. Ketola

D. K. Ploetz

MS -191

Ram Shukla

MS -305

Don Stroud

1

General Accounting Office

P. 0. Box 321

Richland, WA 99352

C. R. Abraham 


\section{Distribution - continued}

Defense Nuclear Facilities Safety Board 625 Indiana Ave, N. W., Suite 700

Washington, D. C. 20004

Dan Burnfield

Lester Clemons

Suite 700

Paul Gubanc

Suite 700

Dermot M. Winters, Geological Engineer

Westinghouse Idaho Nuclear Corporation

P. 0. Box 4000

Idaho Falls, ID 83403-4000

B. Griebenow

MS -5104

A. P. Hoskins

$M S-5217$

1

C. Abrams

1987 Virginia Drive

Idaho Falls, ID 83404

1

F. Carlson

6965 North, 5th West

Idaho Falls, ID 83401

1

Joseph J. DiNunno, Engineering Consultant 44 Carriage Lane

Annapolis, MD 21401

1

D. Oakley

40912 th Street, SW, \#310

Washington, D. C. 20024

1

Dr. A. Veletsos

Department of Civil Engineering

Rice University

P. 0. Box 1892

Houston, TX 77252

Westinghouse Materials Company of Ohio

P. 0. Box 398704

Cincinnati $\mathrm{OH} 45239-8704$

David L. Jacoboski

Senior Engineer, Technology Demonstration

Westinghouse Savannah River Company

P. 0 . Box 616

Aiken, SC 29802

P. d'Entremont 
WHC-EP-0182-56

\section{Distributior, - continued}

Institute for Energy and Environmental Reseach 6935 Laure1 Avenue

Takoma Park, MD 20912

Dr. Arjun Makhijani, President

1

SPAR Aerospace Ltd.

20 Avon Meadow Lane, Suite 220

Avon, CT 06001

Peter W. Kruse

Advanced Technology Systems Division

1

Redzone Robotics, Inc.

2425 Liberty Ave

Pittsburgh, PA 15222-4639

David W. White

12

Nat, inal Research Council. National Academy of Sciences 2101 Constitution Ave., N. W. Washington D. C. 20418

Robert S. Andrews, Senior Staff officer

Board on Radioactive Waste Management

1

Converse Consultants

$18 \mathrm{~W}$. Mercer Street, Suite 300

Seattle, WA 98119

David Stanley

1

Brown \& Caldwell

100 W. Harrison

Seattle, WA 98119

Hal Cooper

1

BovaY Northwest Inc.

660 Swift, Suite D

Richland, WA 99352

T. J. McLaughl in

1

Omega Environmental Technology

655 Montgomery Street, Suite 1000

San Francisco, CA 94111

Mike Bailey

Distr-6 
WHC-EP-0182-56

\section{Distribution - continued}

GEC ALSTHOM Engineering Systems

P. 0. Box 1274

Richland, WA 99352

J. W. Riddington,

Vice President, Nuclear Marketing

1 T. S. Elleman

North Carolina State University

Department of Nuclear Energy

P. 0. Box 7909

Raleigh, NC 27606

1

Mike Lingle

Stone \& Webster

7677 E. Berry Ave

Englewood, CA 80111

1

Bryant Mather

Corps of Engineers

WESSV-Z

3909 Halls Ferry Rd

Vicksburg, MS 39180-6199

1

Paul Shewmon

Prof. Metallurgical Engineer

Ohio State University

2477 Lytham Road

Columbus, $\mathrm{OH} 43220$

Waste Management External Advisory Committee Members

Dr. Frank L. Parker

Professor of Environmental and Water Resources Engineering Vanderbilt University

P. 0. Box 1596, Station B

Nashville, TN 37235

Dr. Bruce R. Kowalski

Professor of Chemistry, Co-director of Center for Process

Analytical Chemistry

University of Washington

Chemistry Department, Bldg 10

Seattle, WA 98195

Dr. Greg R. Choppin

Professor of Chemistry

Florida State University

Department of Chemistry, B-164

Tallahassee, FL 32306 
WHC-EP-0182-56

\section{Distribution - continued}

Dr. Chester Grelecki

President, Chief Scientist

Hazards Research Corporation

200 Valley Road

Mt. Arlington, NJ 07856

Dr. Alfred Schneider

MIT Department of Nuclear Engineering Room 24-1098

77 Massachusetts Avenue

Cambridge, MA 02139

Dr. Gary Powers

President

Design Science, Inc.

163 Witherow Road

Sewickley, PA 15143

Ames Laboratory

7 Spedding Hall

Iowa State University

Ames, IA 50011

Bill Haas

RKK Ltd.

16404 Smokey Pt. Bivd, Suite 303

Arlington, WA 98223

Chris Reno

\section{MACTEC}

8320 Centerbrook Place

Alexandria, VA 22308

Stan Blacker

Engineering-Science, Inc.

1955 Jadwin Ave, Suite 470

Richland, WA 99352

Matt Sakach

EBASCO Services, Inc.

1201 Jadwin Avenue, Suite 202

Richland, WA 99352-3429

F. J. Young

MTL Systems, Inc.

3481 Dayton-Xenia Road

Dayton, OH 45431-0299

E. McDaniel 
WHC-EP-0182-56

\section{Distribution - continued}

Nuclear Consulting Services, Inc.

7000 Hunt ley Road

P. 0. Box 29151

Columbus, $\mathrm{OH} 43229$

Dr. J. Louis Kovach

1

Battelle Laboratories

505 King Avenue

Columbus, $\mathrm{OH}$ 43201-2693

Rob Taylor Jr., P.E.

Rm 13-6-016

1

Portland General Electric Co.

121 S. W. Salmon St.

Portland, OR 97204-2991

Wayne Lei, 3WTCBRO5

\section{OFFSITE - FOREIGN}

1

British Nuclear Fuels Ltd

Risley Warrington

Cheshire WA3 6AS

United Kingdom

Howard A. Edwards

1

Ricardo Hitec Ltd

Club street Horks, Bamber Bridge Preston, PR5 6FN

United Kingdom

P. K. J. Smith

1

Telerobot

Consorzio Telerobot

Via Hermada 6

16154 Genova, Italy

Bruno Sessarego

1

SGN

1 , rue des Herons, Montigny-C-Bretonneux

78132 Saint-Quentin-en-Yvelines Cedex,

France

Serge Merlin

CEA - Saclay 
WHC-EP-0182-56

Distribution - continued

DCC/DIR

$\mathrm{Ba}^{\prime} \mathrm{t} 121$

91190 GIF/Yvette Cedex

France

G. Baudin

R. Atabek

1

Hans Wal ischmiller GmbH

D-7778 Markdorf/Bodensee

Germany

Wolfgang Walischmiller

ONSITE

23

U. S. Department of Energy-Richland Operations Office

G. E. Bishop

K. W. Bracken

R2 -62

G. J. Bracken

A5- 16

S. T. Burnum

R4-02

N. R. Croskrey

A5- 16

R. C. Cullison

R1 -30

J. J. Davis

A5-55

L. Erickson

A5- 16

R. E. Gerton

A5-16

W. F. Hendrickson

A4-02

R. D. Hildebrand

A4-02

P. E. LaMont

A5-55

B. L. Nicoll

A5-16

T. Noble

R. H. Pestes

A5- 16

A4-02

L. E. Petersen

A5-16

G. W. Rosenwald

A5- 10

G. H. Sanders

A4-02

J. B. Sullivan

A5-16

A. D. Toth

W. R. Wresinski

A5-10

R2 -62

A5- 16

J. K. Yerxa

A5-15

Reading Room

Al -65

3

Kaiser Engineering Hanford
C. J. Denson
E6-51
J. E. Fasso Jr.
E2-80
D. J. Shrimpton
E6-21

1

Stone \& Webster Engineering Co.

E. L. Richards

A4-35 
WHC-EP-0182-56

Distribution - continued

J. Janus

G6-18

21

Pacific Northwest Laboratories

D. B. Baird

D. W. Bennett

K7-54

S. A. Bryan

K5-22

L. L. Burger

P7 -25

J. F. Fletcher

P7 -25

L. K. Holton Jr.

K7-97

J. Janata

P7 -43

B. M. Johnson

K2 - 12

E. 0. Jones

$\mathrm{K} 1-78$

L. G. Morgan

P8-38

B. E. Opitz

P7 -35

M. S. Peffers

K6 -81

W. G. Richmond

K7 -94

R. D. Scheele

P7 -41

P. A. Scott

P7 -25

J. C. Spanner

P7 -19

D. Strachan

K2-05

K. L. Steinmaus

$K 2-38$

R. S. Wegeng

K6-84

P. D. Whitney

K7 -97

T. W. Wood

K7 -34

K6-47

Westinghouse Hanford Company

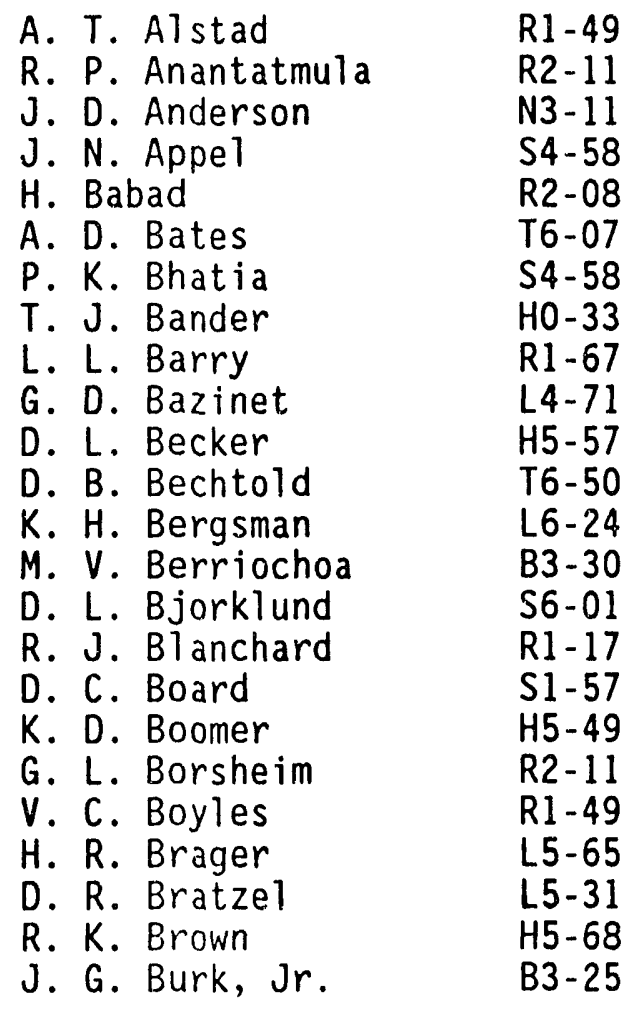




\section{Distribution - continued}

J. H. Bussell

J. A. Caggiano Jr

K. G. Carothers

J. W. Carey

R. J. Cash

G. Christensen

J. C. Conner

F. M. Coony

W. L. Cowley

N. R. Croskrey

G. M. Crummel

J. M. Cruse

D. S. Cunningham

L. T. Cunningham

J. I. Dearing

C. DeFigh-Price

T. A. Demitruk

D. R. Dickinson

E. C. Ladd

R. A. Dodd

M. R. Duncan

G. L. Dunford

W. S. Dunnivant

J. A. Eacker

D. R. Ellingson

M. F. Erhart

S. D. Estey

W. G. Farley

K. 0. Fein

R. A. Flores

L. A. Fort

K. D. Fowler

G. L. Fox, Jr.

G. T. Frater

J. R. Freeman-Pollard

J. C. Fulton

K. A. Gasper

G. J. Gauck

R. L. Gilchrist

D. A. Gilles

S. D. Godfrey

D. J. Green

J. M. Grigsby

V. W. Hall

C. S. Haller

D. G. Hamrick

K. L. Hampsten

B. M. Hanlon (30)

J. M. Hanson

H. D. Harmon

J. P. Harris III

J. M. Henderson

D. W. Hendrickson

N. A. Hertelendy
L7-06

H5 -29

R1 - 51

SO- 01

R2 - 32

$\mathrm{H} 4-21$

H5-31

H6- 07

H5-31

R1-51

R1 - 51

L5-63

SO-02

R1-51

H5-72

R2-31

H5-36

L5-31

R1 -19

R1 - 51

L6- 10

R1-51

R2-88

R1 -51

B5-35

R2-11

R2-11

H5 - 32

H5-34

SO -12

B2 - 20

R2-11

L5-01

R1-51

H6-03

R2-31

R2 - 08

R2 - 07

L5-63

S2-70

R1-51

H5-53

H4-62

Bl - 59

R2 - 18

R1 - 51

L4 - 71

R1-80

R2 - 40

R2 - 52

S4 -55

S4-55

R4-03

H5-09 
E. G. Hess

M. C. Higginson

J. G. Hill

B. M. Hisaw

J. D. Hopkins

B. K. Horsager

R. D. House

J. H. Huber

J. L. Huckaby

L. L. Humphreys

J. E. Hysjulien

J. E. Irwin

M. N. Is I am

G. D. Johnson

J. L. Juette

L. J. Julyk

R. A. Kirkbride

N. W. Kirch

W. L. Knecht

E. M. Koellermeier

G. M. Koreski

A. G. Krasopoulos

M. Kummerer

M. J. Kupfer

J. E. Langdon

D. L. Lenseigne

D. C. Lini

P. J. Mackey

G. T. Maclean

M. K. Mahaffey

R. M. Marusich

V. D. Maup in

T. B. McCall

J. D. McCormack

K. S. McCullough

M. E. McDonald

T. E. Mensinger

N. J. Milliken

W. J. Millsap

G. J. Miskho

J. R. Mobley

K. L. Morris

J. P. Mullally

L. D. Muhlestein

A. F. Noonan

T. W. Oden

P. C. Oh 1

D. B. Pabst

R. B. Pan

I. G. Papp

G. L. Parsons

M. A. Payne

T. B. Powers

R. K. P'Pool
R3-09

A4- 25

R2 - 12

R1 -62

R2-11

S4 -57

R2 - 83

R1 -49

R2-11

R2 - 50

SO- 09

B1-59

R3-08

$\mathrm{R} 2-78$

G6- 56

H5-56

S4-58

R2-11

HO -34

R4 - 40

R1 - 51

A5-55

H5-32

H5- 49

S2-96

R2 - 75

H5 -49

B3-15

S4 - 58

L4-73

H5 - 32

R1 - 51

HO- 33

L5-31

H5-34

R1-62

T3-10

H5-34

H5-68

R2-50

R2-18

H5- 09

S4-57

N1-28

R2- 12

D2 -29

R1 -30

B2 - 35

H5 -53

R3-45

S4-57

R2-50

HO- 31

T1-30 


\section{WHC-EP-0182-56}

\section{Distribution - continued}

J. G. Propson

T. E. Rainey

R. E. Raymond (2)

R. W. Reed

I. E. Reep

M. A. Rezvani

D. Richardson

J. H. Roecker

L. Ruffin

J. A. Ryan

C. C. Scaief

F. A. Schmorde

J. S. Schofield

C. P. Schroeder

D. D. Scott

K. V. Scott

R. A. Shea

J. E. Shapley

E. M. Sheen

E. J. Shen

P. K. Shen

A. T. Shook

A. B. Sidpara

S. G. Spencer

S. M. Stahl

E. G. Stephan

R. R. Stickney

T. L. Strathman

J. N. Strode

K. C. Strong

M. J. Sutey

D. G. Sutherland

J. P. Summerhays

L. M. Swanson

J. F. Thompson

J. D. Thoilson

S. R. Tifft

J. A. Tilden (2)

H. Toffer

T. T. Tran

J. B. Truitt

R. E. Vandercook

R. J. Van Vleet

J. A. Voogd

J. R. Weber

D. L. Wegener

R. K. Welty

G. T. Wells

K. A. White

D. D. Wodrich
R2 - 18

R1 -49

Rl -80

R1-51

R2 - 08

H5 - 55

R2-31

G6-02

R2-95

H5-55

L7- 06

R2-88

R1-51

L7-06

L6-10

H5-52

SO-02

N1-83

L7 -05

S4-58

HO-39

S2-01

A2-91

R1 -30

H5-31

A3 -74

R1-49

SO- 14

R1-51

R2-12

R1-49

L4 - 72

R2-88

H5- 34

H5-71

R1-30

H6-26

L6-10

HO-38

L7-05

H5-56

S6-07

H5-32

R4-03

G6-46

R1-62

R] -80

H6-26

R1-51

BI - 59 
WHC-EP-0182-56

Distribution - continued

Docket File (2) H5-36

272-AW Shift Office S5-04

Central Files (2) L8-0.4

Tank Farms Info Center R1-28

Information Release

Administration (3) R1-08

Env Data Mgmt Center H4-22 

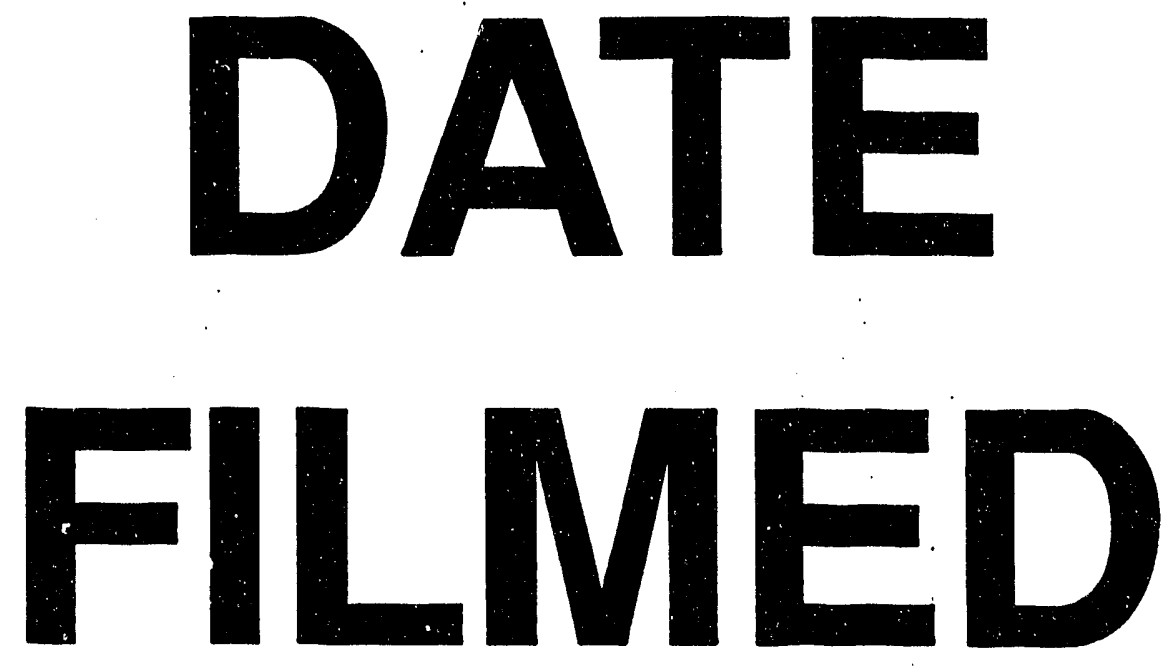

$8 / 17 / 93$
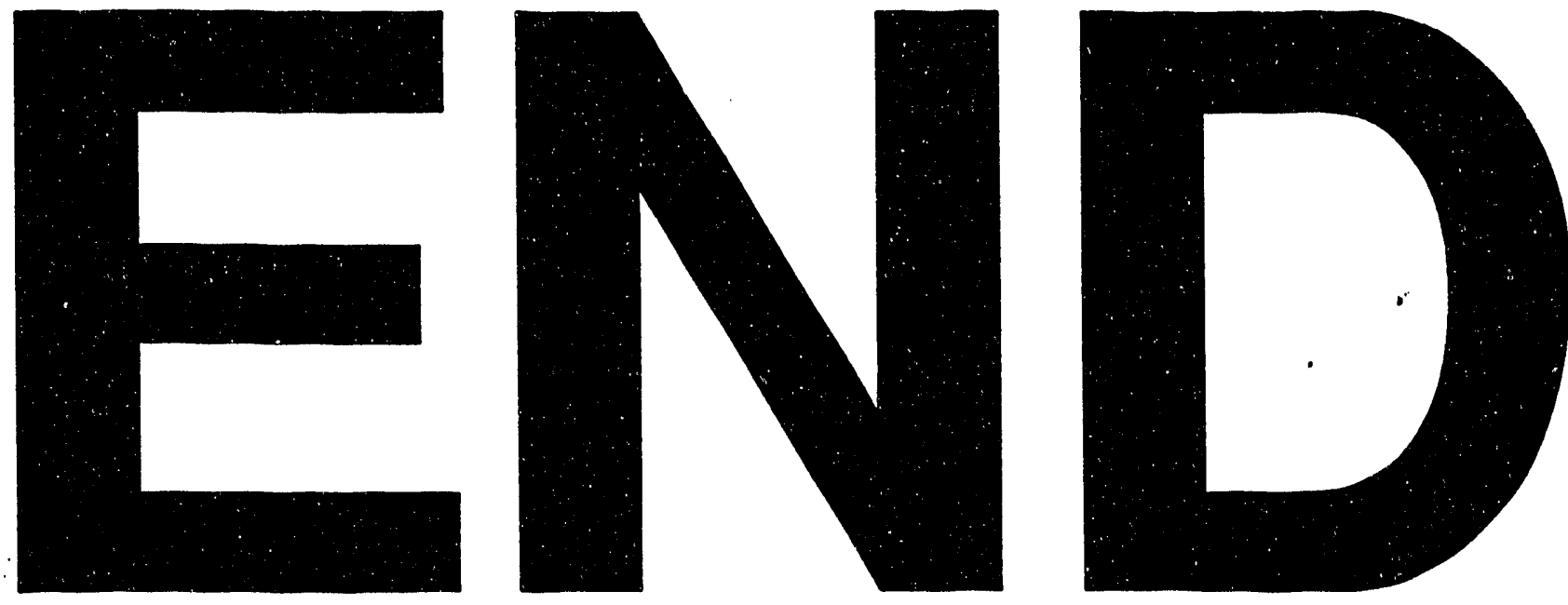
